\title{
Search for low-scale gravity signatures in multi-jet final states with the ATLAS detector at $\sqrt{s}=8 \mathrm{TeV}$
}

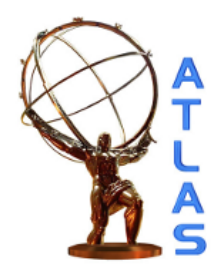

\section{The ATLAS collaboration}

\section{E-mail: atlas.publications@cern.ch}

ABstract: A search for evidence of physics beyond the Standard Model in final states with multiple high-transverse-momentum jets is performed using $20.3 \mathrm{fb}^{-1}$ of proton-proton collision data at $\sqrt{s}=8 \mathrm{TeV}$ recorded by the ATLAS detector at the LHC. No significant excess of events beyond Standard Model expectations is observed, and upper limits on the visible cross sections for non-Standard Model production of multi-jet final states are set. A wide variety of models for black hole and string ball production and decay are considered, and the upper limit on the cross section times acceptance is as low as $0.16 \mathrm{fb}$ at the $95 \%$ confidence level. For these models, excluded regions are also given as function of the main model parameters.

KEYwords: Exotics, Hadron-Hadron Scattering

ARXIV EPRINT: 1503.08988 


\section{Contents}

1 Introduction 1

2 Theoretical background and previous results 2

3 ATLAS detector 3

4 Monte Carlo simulation 4

5 Trigger and data selection 5

6 Background estimation method 5

$\begin{array}{lll}7 & \text { Systematic uncertainties } & 9\end{array}$

$\begin{array}{lll}8 & \text { Results } & 10\end{array}$

9 Conclusion $\quad 18$

The ATLAS collaboration $\quad 22$

\section{Introduction}

Most models of low-scale gravity allow the production of non-perturbative gravitational states, such as micro black holes and string balls (highly excited string states) at Large Hadron Collider (LHC) collision energies [1-4]. This is due to the fundamental gravitational scale being comparable to the electroweak scale $\left(M_{\mathrm{EW}}\right)$ in these gravity models. If black holes or string balls are produced at the LHC with masses much higher than this fundamental gravitational scale, they behave as classical thermal states and decay to a relatively large number of high-transverse-momentum (high- $p_{\mathrm{T}}$ ) particles. One of the predictions of these models is the expectation that particles are emitted from black holes primarily according to the number of Standard Model (SM) degrees of freedom (number of charge, spin, flavour, and colour states).

To identify high- $p_{\mathrm{T}}$, high-multiplicity final states resulting from high-mass objects, a suitable variable is the scalar sum of the $p_{\mathrm{T}}$ of the jets in the event, $H_{\mathrm{T}}$. A low- $H_{\mathrm{T}}$ control region is defined where the background is expected to dominate over any possible new physics signal. A fit-based technique is used to extrapolate from the control region to a high- $H_{\mathrm{T}}$ signal region to estimate the amount of SM background.

This paper is organised as follows. The phenomenology of low-scale gravity relevant to the search is briefly described in section 2 . In section 3 , the main components of the ATLAS detector are summarised. The Monte Carlo (MC) simulated samples used for 
the analysis are presented in section 4 . In section 5, the trigger and event selection are described. The characterisation of the data and the method used in the search are given in section 6 . Section 7 describes the systematic uncertainties, and the resulting limits are given in section 8. Finally, conclusions are stated in section 9.

\section{Theoretical background and previous results}

Understanding quantum gravity is one of the main challenges of modern physics. The hierarchy problem (the relative weakness of gravity compared to the electroweak interaction) may be key to that understanding. Two main paradigms for models involving extra dimensions have been formulated: the Arkani-Hamed, Dimopoulos, Dvali (ADD) proposal [1, 2] involving large extra dimensions; and a five-dimensional model with a single highly warped anti-de Sitter space $[3,4]$. These models have our $(3+1)$-dimensional world residing on a brane, which is embedded in a $(4+n)$-dimensional bulk with $n$ extra dimensions. The effective strength of the gravitational interaction inside the brane is weakened by the large volume of the extra dimensions or red-shifted by the warp factor along the extra dimension. This weakening of the gravitational strength results in a diminished effective Planck scale $M_{D}$ in the $(4+n)$-dimensional world, relative to the familiar Planck scale $M_{\mathrm{Pl}}$. In the ADD model, there are a number $n>1$ additional flat extra dimensions, and $M_{D}$ is determined by the volume and shape of the extra dimensions.

If $M_{D} \sim M_{\mathrm{EW}}$, several low-scale gravitational signatures may be probed in collider physics experiments. Some of the most interesting are the possible existence of nonperturbative gravitational states such as black holes [1-4], string balls [5] (in the context of weakly coupling string theory), and higher-dimensional branes.

Within the context of the ADD model, experimental lower limits on the value of $M_{D}[6]$ were obtained from experiments at LEP and the Tevatron [7, 8], as well as at ATLAS [9] and CMS [10], by searching for the production of the heavy Kaluza-Klein gravitons associated with the extra dimensions. The most stringent limits come from the LHC analyses $[9,10]$ that search for non-interacting gravitons recoiling against a single jet, and range from $M_{D}>3.1 \mathrm{TeV}$, for $n=6$, to $M_{D}>5.2 \mathrm{TeV}$, for $n=2$. Several searches for black holes and string balls are also performed by ATLAS [11-14] and CMS [15-17].

In proton-proton collisions with centre-of-mass energy $\sqrt{s}$, classical black holes form when the impact parameter between two colliding partons, with centre-of-mass energy $\sqrt{\hat{s}}$, is less than twice the gravitational radius $r_{\mathrm{g}}$ of a black hole of mass equal to $\sqrt{\hat{s}}[18,19]$. Black holes are assumed to be produced over a continuous range of masses above a certain threshold $M_{\mathrm{th}} \gtrsim M_{D}$ up to $\sqrt{s}$. Semi-classical approximations used in the modelling are valid for masses only well above $M_{D}$, motivating the use of a minimal threshold $M_{\text {th }}$ to remove contributions where the modelling is not reliable.

Most low-scale gravity models assume classical general relativity to predict the production cross section for black holes $\left(\sigma \sim \pi r_{\mathrm{g}}^{2}\right)$ and string balls, and use semi-classical Hawking evaporation (a completely thermal process due to quantum effects) to describe their decay [20]. The decay process is described by black-body radiation at the Hawking temperature (Hagedorn temperature for string balls) with the expectation that the radi- 
ated particle species are produced according to the number of SM degrees of freedom and are not affected by the strengths of the SM forces. The emissions are modified by spindependent quantum statistics given by the Fermi-Dirac or Bose-Einstein distributions. In addition, the emissions are modified by gravitational transmission factors [20] (gray-body factors), which depend on the spin of the emitted particle, as well as the angular momentum of the black hole, and can be sizeable for vector particle emission from rotating black holes. Once black holes are produced, they evaporate causing their mass to be reduced with each emitted particle. In the context of weakly coupled string theory, black holes transition to string balls at a minimum black hole mass $M_{\min } \sim M_{\mathrm{s}} / g_{\mathrm{s}}^{2}$, where $M_{\mathrm{S}}$ is the string scale and $g_{\mathrm{s}}$ is the string coupling constant [5, 21]. When the black hole mass is reduced to approximately $M_{D}$ (or $M_{\mathrm{s}}$ for string balls), the black hole is said to be in a remnant state, which is expected to only be describable by a theory of quantum gravity. This study only considers unstable black hole remnants, and black holes and string balls that are short lived.

The production and decay of black holes and string balls lead to final states distinguished by a high multiplicity of high- $p_{\mathrm{T}}$ particles, consisting mostly of jets arising from quark and gluon emission. Since black hole decay is considered to be a stochastic process, a different number of particles, and thus jets, can be emitted from black holes with identical kinematics.

\section{ATLAS detector}

The ATLAS experiment [22] is a multi-purpose particle physics detector with a forwardbackward symmetric cylindrical geometry and nearly $4 \pi$ coverage in solid angle. ${ }^{1}$ The layout of the detector is dominated by four superconducting magnet systems, which comprise a thin solenoid surrounding inner tracking detectors and three large toroids, each consisting of eight coils. The inner detector consists of a silicon pixel detector, a silicon microstrip detector, and a transition radiation tracker, with a combined coverage up to $|\eta|=2.5$. In the pseudorapidity region $|\eta|<3.2$, liquid-argon (LAr) electromagnetic (EM) sampling calorimeters are used. An iron/scintillator tile calorimeter provides hadronic coverage over $|\eta|<1.7$. The end-cap and forward regions, spanning $1.5<|\eta|<4.9$, are instrumented with LAr calorimetry for EM and hadronic measurements. The muon spectrometer surrounds these, and comprises a system of precision tracking and trigger chambers. A three-level trigger system is used to select interesting events [23]. The Level-1 trigger is implemented in hardware and uses a subset of detector information to reduce the event rate to at most $75 \mathrm{kHz}$. This is followed by two software-based trigger levels which together reduce the event rate to about $300 \mathrm{~Hz}$.

\footnotetext{
${ }^{1}$ The ATLAS detector uses a right-handed coordinate system with its origin at the nominal interaction point in the centre of the detector and the $z$-axis along the beam direction. The $x$-axis points toward the centre of the LHC ring, and the $y$-axis points upward. Cylindrical coordinates $(r, \phi)$ are used in the transverse plane, $\phi$ being the azimuthal angle around the beam pipe. The pseudorapidity $\eta$ is defined in terms of the polar angle $\theta$ by $\eta \equiv-\ln [\tan (\theta / 2)]$.
} 


\section{Monte Carlo simulation}

All background estimates in this analysis are derived from data. However, SM MC simulated events are used to estimate the relative background contributions from different processes expected in the data sample, and to develop and validate the analysis methods.

The dominant background in the search region consists of QCD multi-jet events, with small contributions from top quark pair production $(t \bar{t}), \gamma+$ jets, $W+$ jets, and $Z+$ jets. Single-top-quark and diboson processes contribute negligibly to the selected samples. The baseline samples of inclusive jets are generated using PYTHIA 8.160 [24] implementing LO perturbative QCD matrix elements for $2 \rightarrow 2$ processes and $p_{\mathrm{T}}$-ordered parton showers calculated in a leading-logarithmic approximation. The ATLAS AU2 set of MC parameters (tune) [25] and the CT10 [26] PDFs are used with these samples. Herwig++ 2.6 .3 [27] dijet samples with the ATLAS EE3 tune and CTEQ6L1 [28] PDFs, and ALPGEN 2.14 [29] multijet samples hadronised with PYTHIA 6.427 with the ATLAS Perugia 2001C tune and the CTEQ6L1 PDFs are used for comparisons. The $t \bar{t}, \gamma+$ jets, $W+$ jets, and $Z+$ jets samples are generated using SHERPA 1.4.0 [30] with CT10 PDFs. All MC simulated background samples (except ALPGEN) are using the full GEANT4 [31] simulation.

Signal acceptances are determined using MC simulated events. Signal samples are generated using the MC event generators CHARYBDIS2 1.0.2 [32] and BlackMax 2.02.0 [33]. Two generators are used since they model the remnant decay slightly differently and neither implements all the models considered in this analysis. CHARYBDIS2 is used to produce samples for non-rotating, rotating, and low-multiplicity remnant black holes, and for an initial-state graviton radiation model. BlackMax is used to produce samples for non-rotating and rotating black holes, and for final-state graviton emission and initial-state photon radiation models. The initial-state radiation is modelled to occur after, rather than before, black hole formation. In addition, CHARYBDIS2 is used to produce non-rotating and rotating string ball samples. Both generators use a leading-order parton distribution function (PDF) MSTW2008 [34], the ATLAS AU2 tune, and the PYTHIA 8.165 generator for fragmentation. The most important parameters that have significant effects on black hole production are $M_{\mathrm{th}}, M_{D}$ ( $M_{\mathrm{s}}$ for string balls), and $n$. Signal samples are produced for many values of these parameters. The MC simulated signal samples are passed through a fast simulation of the ATLAS detector [35]. The fast simulation uses a parameterised response of the calorimeters, and GEANT4 for the other parts of the ATLAS detector. The difference in signal yield with respect to a full GEANT4 simulation of the ATLAS detector [36] is negligible.

Additional proton-proton collisions are modelled by overlaying minimum bias events on the simulated signal and background events according to the luminosity profile of the recorded data. The MC simulated events are reconstructed and analysed with the same procedures as used on data. 


\section{$5 \quad$ Trigger and data selection}

The data used in this analysis were recorded in 2012, with the LHC operating at a centreof-mass energy of $\sqrt{s}=8 \mathrm{TeV}$. All detector elements are required to be fully operational, and a total integrated luminosity of $20.3 \mathrm{fb}^{-1}$ is used in this analysis with a luminosity uncertainty of $2.8 \%$. It is derived following the same methodology as that detailed in ref. [37].

The events used in this search are selected using a high- $H_{\mathrm{T}}$ trigger, which requires at least one jet of hadrons with $p_{\mathrm{T}}>170 \mathrm{GeV}$ and a high scalar sum of transverse momentum of all the jets in the event. The trigger is fully efficient if the event has $H_{\mathrm{T}}>1.2 \mathrm{TeV}$, as required in this analysis.

Events are required to have a primary vertex with at least two associated tracks with $p_{\mathrm{T}}$ above $400 \mathrm{MeV}$. The primary vertex assigned to the hard scattering collision is the one with the highest $\sum_{\text {track }} p_{\mathrm{T}}^{2}$, where the scalar sum of track $p_{\mathrm{T}}^{2}$ is taken over all tracks associated with that vertex.

Since black holes and string balls are expected to decay predominantly to quarks and gluons, the search is simplified by considering only jets. The analysis uses jets of hadrons, as well as misidentified jets from photons, electrons, and $\tau$ leptons. The incorrect calibration of photons, electrons, and $\tau$ leptons using the hadronic energy calibration leads to small energy shifts for these particles, but since a particle of this type is expected to occur in less than $0.6 \%$ (as determined from simulation studies) of the events in the data sample, they do not contribute significantly to the resolution of global quantities.

The anti- $k_{t}$ algorithm [38] is used for jet finding, with a radius parameter $R=0.4$. The inputs to the jet reconstruction are three-dimensional topo-clusters [39]. This method first clusters together topologically connected calorimeter cells and then classifies these clusters as either electromagnetic or hadronic. The classification uses a local cluster weighting calibration scheme based on cell-energy density and longitudinal depth within the calorimeter. Based on this classification, energy corrections described in ref. [40] are applied. Furthermore, jets are corrected for pile-up. The jets are required to have $p_{\mathrm{T}}>50 \mathrm{GeV}$ and $|\eta|<2.8$ in this analysis.

\section{Background estimation method}

Events are selected if they pass the high- $H_{\mathrm{T}}$ trigger and have $H_{\mathrm{T}}>1.5 \mathrm{TeV}$. The discriminating variable chosen for this analysis is $H_{\mathrm{T}}$. Figure 1 shows the $H_{\mathrm{T}}$ distributions for different inclusive jet multiplicities. Data as well as MC simulations of the most significant SM contributions to the $H_{\mathrm{T}}$ distributions are shown. The different $\mathrm{MC}$ contributions are first weighted according to their cross sections, and then the total SM contribution is normalised to the number of data events in the region $1.5<H_{\mathrm{T}}<2.9 \mathrm{TeV}$ for each inclusive jet multiplicity. As can be seen in figure 1, the expected SM background is dominated by QCD jet production. The rest of the background processes contribute less than $3 \%$ to the total background, and therefore the other contributions are neglected in what follows. 

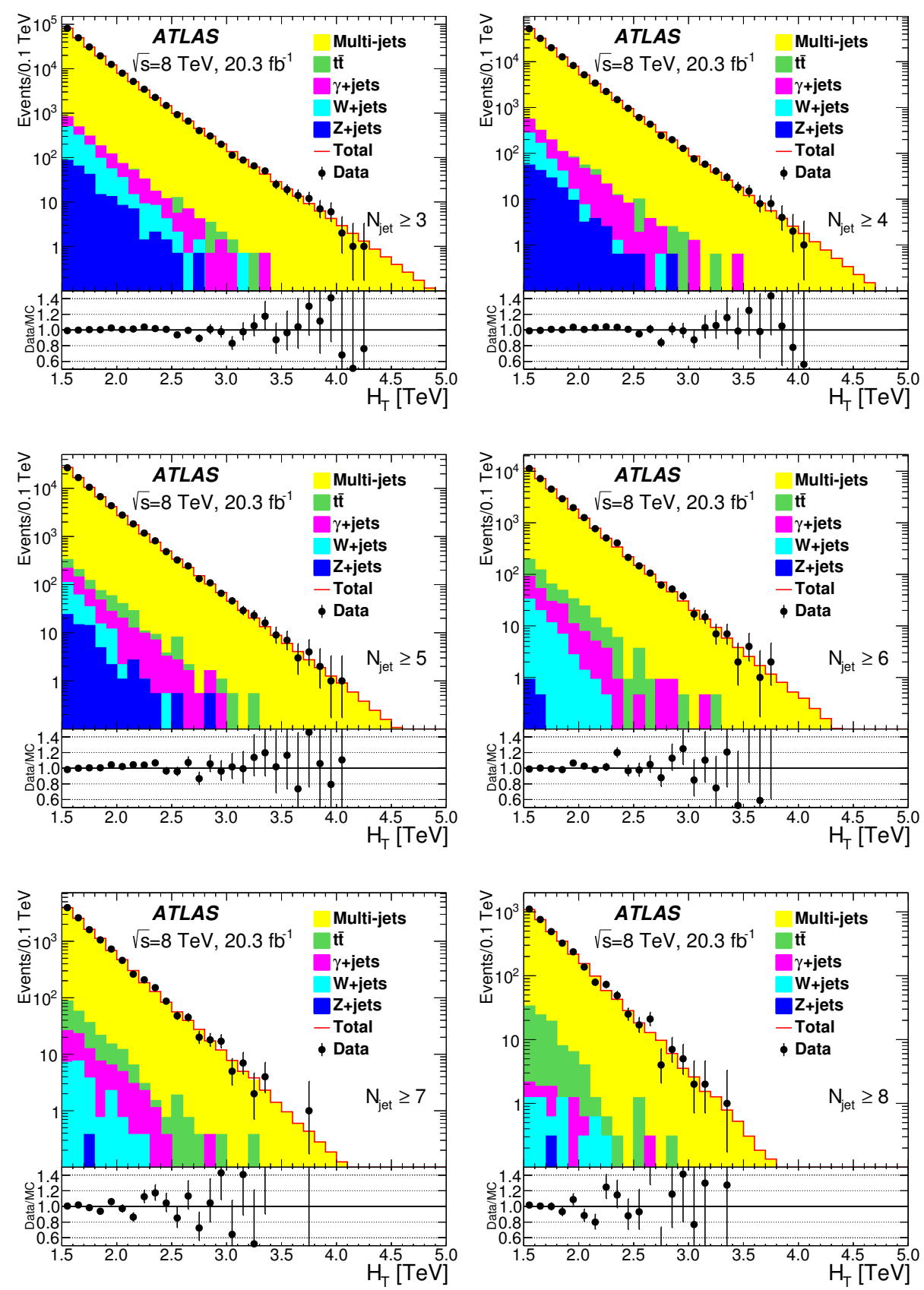

Figure 1. Distributions of the scalar sum of the $p_{\mathrm{T}}$ of all jets in the event, $H_{\mathrm{T}}$, for different inclusive jet multiplicities $N_{\text {jet }}$ for $20.3 \mathrm{fb}^{-1}$ of collision data and MC simulations of SM processes. The uncertainties on the data and ratio points are due to the statistical uncertainty of the data only. The SM contributions to the background are normalised relative to their nominal cross sections and then the total background is normalised to the number of data events in the region $1.5<H_{\mathrm{T}}<2.9 \mathrm{TeV}$ for each inclusive jet multiplicity.

Figure 1 also shows good agreement between SM expectations and data, which is quantified in section 8. 
Each event is characterised by the number of jets $N_{\text {jet }}$ and by the value of $H_{\mathrm{T}}$. The $\left(N_{\text {jet }}, H_{\mathrm{T}}\right)$-variable space is divided into two exclusive $H_{\mathrm{T}}$ regions for the search, which are each further divided by inclusive jet multiplicity $N_{\text {jet }}$. The two exclusive $H_{\mathrm{T}}$ regions are defined as a control region $\left(1.5<H_{\mathrm{T}}<2.9 \mathrm{TeV}\right)$ and as a signal region $\left(H_{\mathrm{T}}>3.0 \mathrm{TeV}\right)$, for each inclusive jet multiplicity. The control region is utilised to fit the $H_{\mathrm{T}}$ distribution, as no resonances or threshold enhancements above SM processes were observed in this region. The signal region is the kinematic region in which data are compared to the extrapolation from the control region to search for enhancements. The search is divided into six overlapping regions of inclusive jet multiplicity: $N_{\text {jet }} \geq 3$ to $N_{\text {jet }} \geq 8$. The region with less than three jets is excluded because non-perturbative gravitational states are unlikely to decay to one or two jets [19] and the SM background would be larger with respect to the signal than in the higher jet-multiplicity regions. Other ATLAS searches [41] have set limits on this low-multiplicity region.

The SM background in each signal region is estimated by fitting a function to the data in the corresponding control region and then extrapolating the resulting function to the signal region. To fit the $H_{\mathrm{T}}$ distribution, a three-parameter $p_{0}, p_{1}, p_{2}$ empirical function

$$
\frac{\mathrm{d} N}{\mathrm{~d} H_{\mathrm{T}}}=\frac{p_{0}(1-x)^{p_{1}}}{x^{p_{2}}}
$$

where $x \equiv H_{\mathrm{T}} / \sqrt{s}$, is used.

The selection of the boundaries of the control region is based on 1) stability of the extrapolation into the signal region of the function fit to data with respect to small changes in the choice of control region, and 2) minimisation of possible black hole (or string ball) signal contamination in the control region.

The effect of possible signal contamination in the control region was studied. A string ball sample $\left(n=6, M_{\mathrm{th}}=4.5 \mathrm{TeV}, M_{\mathrm{s}}=1.0 \mathrm{TeV}\right.$, and $\left.g_{\mathrm{s}}=0.4\right)$ was used in this study since it had the largest fraction of events in the control region. The number of string ball events was scaled down (by a factor of 27) until its contribution to the signal region was at the current level of detectability (three standard deviations above the expected background). It was determined that this level of signal would not affect the fit and its extrapolation by more than the statistical uncertainty if the upper boundary on the control region is below $2.9 \mathrm{TeV}$. The value of the cross section used in this study has already been ruled out at the $95 \%$ confidence level (CL) [11-17].

Although the background estimate only relies on data, the validity of the assumption that the fit in the control region can be used to estimate the background in the signal region was tested using PYTHIA 8, Herwig++, and ALPGEN MC simulated events. Since the multi-jet SM simulated events do not contain a signal, the results of the fit to the entire $H_{\mathrm{T}}$ distribution can be compared to the results of the fit to only the control region and extrapolating into the signal region. As an example, the results for PYTHIA 8 dijet simulated events is shown in figure 2. These studies show that the fit extrapolation approximates the MC simulated events in the signal region to within $20 \%$. This difference is covered by the uncertainties, which are described in the next section. 

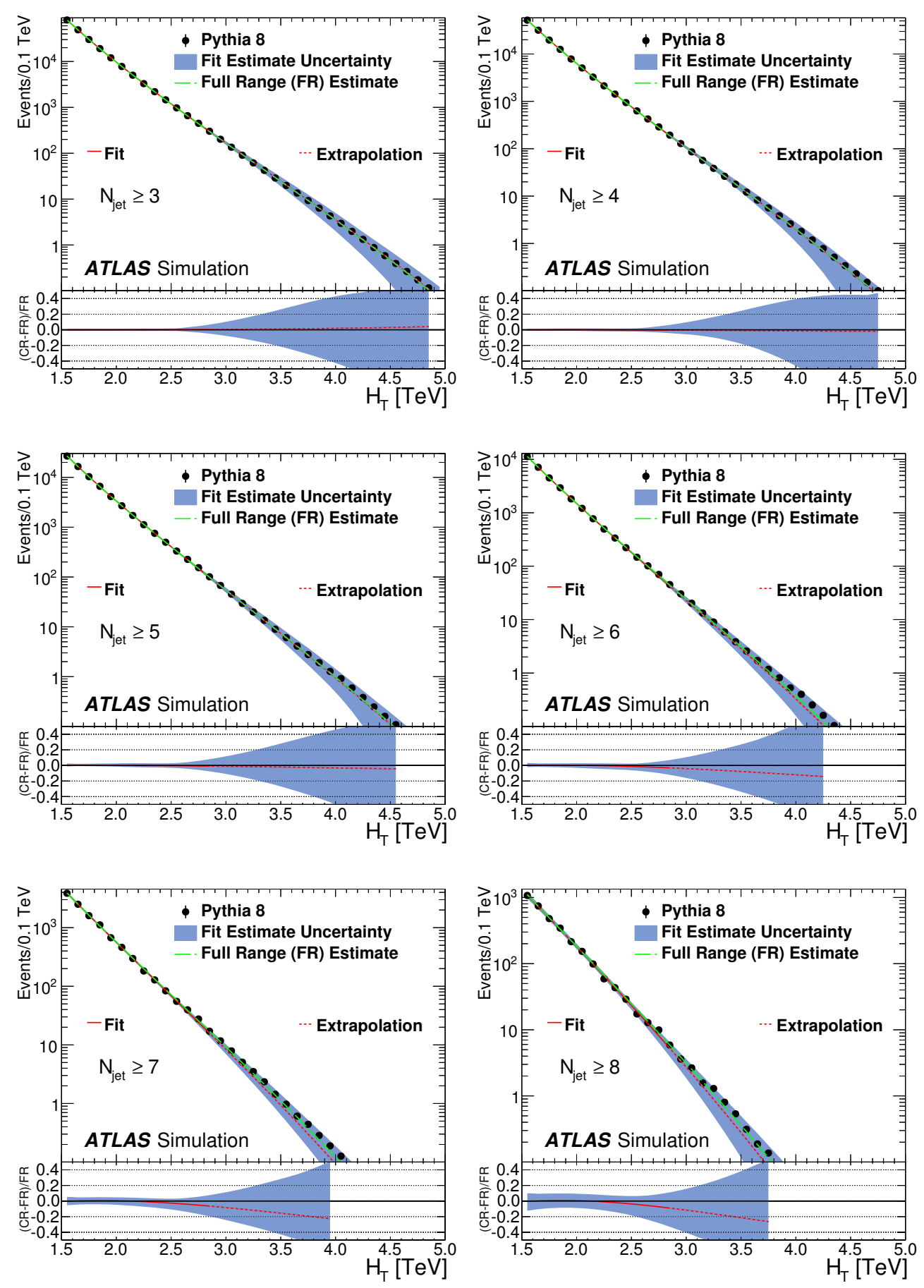

Figure 2. The $H_{\mathrm{T}}$ distributions, showing a comparison of the full range (FR) fit from $H_{\mathrm{T}}=1.5 \mathrm{TeV}$ to the last predicted data value with the extrapolation of the fit from the control region $(\mathrm{CR})$ $1.5<H_{\mathrm{T}}<2.9 \mathrm{TeV}$ into the signal region $\left(H_{\mathrm{T}}>3.0 \mathrm{TeV}\right)$ for PYTHIA 8 simulated events. The uncertainty band includes all the uncertainties described in section 7 . 


\section{$7 \quad$ Systematic uncertainties}

In addition to the statistical uncertainties from the limited number of events in the control region, systematic uncertainties arising from the choice of control region and the choice of fit function are considered. To estimate the effect of limited number of events in the $H_{\mathrm{T}}$ distributions on the fit, pseudo $H_{\mathrm{T}}$-distributions with the same number of events as data are generated using the fit to data in the control region as a probability density function. Each pseudo $H_{\mathrm{T}}$-distribution is fit, and the predicted number of events in each $H_{\mathrm{T}}$ bin is calculated and subtracted from the prediction from the fit to data. A distribution of these differences between the fit to data and the fit to pseudo distributions is used to derive an uncertainty on the fit in each $H_{\mathrm{T}}$ bin. The value of the deviation corresponding to $68 \%$ of the area, about the nominal fit prediction, under the distribution is taken as the asymmetric statistical uncertainty on the fit. In the signal regions, the statistical uncertainty on the fit rises from $5 \%$ at the lower edge of the $H_{\mathrm{T}}$ range to $17 \%$ at the limit of no data for $N_{\text {jet }} \geq 3$, and from $37 \%$ to $67 \%$ for $N_{\text {jet }} \geq 8$.

A systematic uncertainty is assigned due to the $H_{\mathrm{T}}$ range chosen for the control regions. To estimate the uncertainty on the nominal choice, the data are fit in all eight possible $H_{\mathrm{T}}$ control regions by increasing and decreasing the fit range by $0.1 \mathrm{TeV}$, and shifting it by $\pm 0.1 \mathrm{TeV}$. Each fit is used to predict the number of events in each $H_{\mathrm{T}}$ bin. The control region predicting the largest number of events and the control region predicting the smallest number of events for each $H_{\mathrm{T}}$ bin provide an estimate of the asymmetric uncertainty due to the choice of control region. In the signal regions, the systematic uncertainty due to the choice of control region rises from $2 \%$ at the lower edge of the $H_{\mathrm{T}}$ range to $8 \%$ at the limit of no data for $N_{\text {jet }} \geq 3$, and from $28 \%$ to $53 \%$ for $N_{\text {jet }} \geq 8$.

To estimate the uncertainty in the analysis due to the choice of fit function, alternative fit functions are considered. Alternative fit functions are chosen such that in multi-jet simulated events (PYTHIA 8 and Herwig++) they provide a good fit in the control region and the extrapolation of the fit provides a good description of the simulated events in the corresponding signal region. In addition, the function should also provide a good description of the data in the control region. Only functional forms that fulfil these criteria for at least one inclusive jet multiplicity region are considered, and these are:

$$
\begin{aligned}
& p_{0}(1-x)^{p_{1}} e^{p_{2} x^{2}}, \\
& p_{0}(1-x)^{p_{1}} x^{p_{2} x}, \\
& p_{0}(1-x)^{p_{1}} x^{p_{2} \ln x}, \\
& p_{0}(1-x)^{p_{1}}(1+x)^{p_{2} x}, \\
& p_{0}(1-x)^{p_{1}}(1+x)^{p_{2} \ln x}, \\
& \frac{p_{0}}{x}(1-x)^{\left[p_{1}-p_{2} \ln x\right]}, \\
& \frac{p_{0}}{x^{2}}(1-x)^{\left[p_{1}-p_{2} \ln x\right]} .
\end{aligned}
$$

The systematic uncertainty due to the choice of fit function is assigned as the envelope of all alternative fit functions around the nominal function when fit to data in the control 
region. Figure 3 shows the alternative fit functions for different inclusive jet multiplicities. In the signal regions, the systematic uncertainty due to the choice of fit function rises from $10 \%$ at the lower edge of the $H_{\mathrm{T}}$ range to $46 \%$ at the limit of no data for $N_{\text {jet }} \geq 3$, and from $6 \%$ to $10 \%$ for $N_{\text {jet }} \geq 8$.

The jet energy scale (JES) uncertainties and jet energy resolution (JER) uncertainty are used in the MC validation methods and applied to the MC signal samples for the model-dependent limit calculations only. In the signal regions, the JES uncertainty rises from $1 \%$ at the lower edge of the $H_{\mathrm{T}}$ range to $22 \%$ at the limit of no data, and the JER uncertainty rises from $0.6 \%$ to $2 \%$ for $N_{\text {jet }} \geq 3$. For $N_{\text {jet }} \geq 8$, the JES uncertainty rises from $21 \%$ at the lower edge of the $H_{\mathrm{T}}$ range to $34 \%$ at the limit of no data, and the JER uncertainty rises from $7 \%$ to $11 \%$.

\section{Results}

Figure 4 shows the extrapolation of the fits in the control region to the signal region with all uncertainties included. No data events are observed above $H_{\mathrm{T}}=4.3 \mathrm{TeV}$, in agreement with the background estimate.

To test the consistency of the data with the null-hypothesis (background-only hypothesis) a hyper-test statistic $t=-\ln \left[p\right.$-value $\left.{ }^{\mathrm{min}}\right]$ is defined where $p$-value ${ }^{\text {min }}$ is the minimum local $p$-value in any inclusive $N_{\text {jet }}$ and $H_{\mathrm{T}}$ region [42]. The $H_{\mathrm{T}}$ regions can range from a single $0.1 \mathrm{TeV}$ bin to the entire range containing data. The hyper-test statistic takes account of the trials factor (look-elsewhere effect). The most significant discrepancy in the observed signal region distributions is an excess in the interval $3.2 \mathrm{TeV}$ to $3.9 \mathrm{TeV}$, for $N_{\text {jet }} \geq 4$. This enhancement corresponds to a local $p$-value of 0.0043 which corresponds to a significance of 2.6 standard deviations [43] compared to the most probable value for the null-hypothesis of $2.3 \sigma$. The corresponding $t$-value for the data is 5.4 , and for the null-hypothesis the probability to find a value equal or greater than 5.4 is 0.4 . This test shows that no significant excess is observed beyond the SM expectations for all choices of $H_{\mathrm{T}}$ and inclusive $N_{\text {jet }}$ signal regions.

Since black holes or string balls are likely to appear as an enhancement in the tail of $H_{\mathrm{T}}$ distributions, rather than as resonances, production upper limits are set in bins of inclusive $H_{\mathrm{T}}\left(H_{\mathrm{T}}^{\min }\right)$, rather than $H_{\mathrm{T}}$. The predicted number of events in each $H_{\mathrm{T}}^{\min }$ bin in the signal region is obtained by integrating the fit function from the $H_{\mathrm{T}}$ bin of interest up to the kinematic limit of $8 \mathrm{TeV}$. The same integral is performed for the maximum and minimum number of predicted events obtained from the statistical uncertainty and each systematic uncertainty. The differences between the maximum (or minimum) number of predicted events and the nominal number of predicted events for each uncertainty are added linearly to obtain the total uncertainty on the number of predicted events in each $H_{\mathrm{T}}^{\min }$ bin. The uncertainties are added linearly since each is obtained from the same data in the control region.

Using the observed number of events in each signal region compared to the predicted number of SM background events, model-independent limits on the observation of new phenomena at high $H_{\mathrm{T}}$ and inclusive $N_{\text {jet }}$ are set. In addition, model-dependent limits 

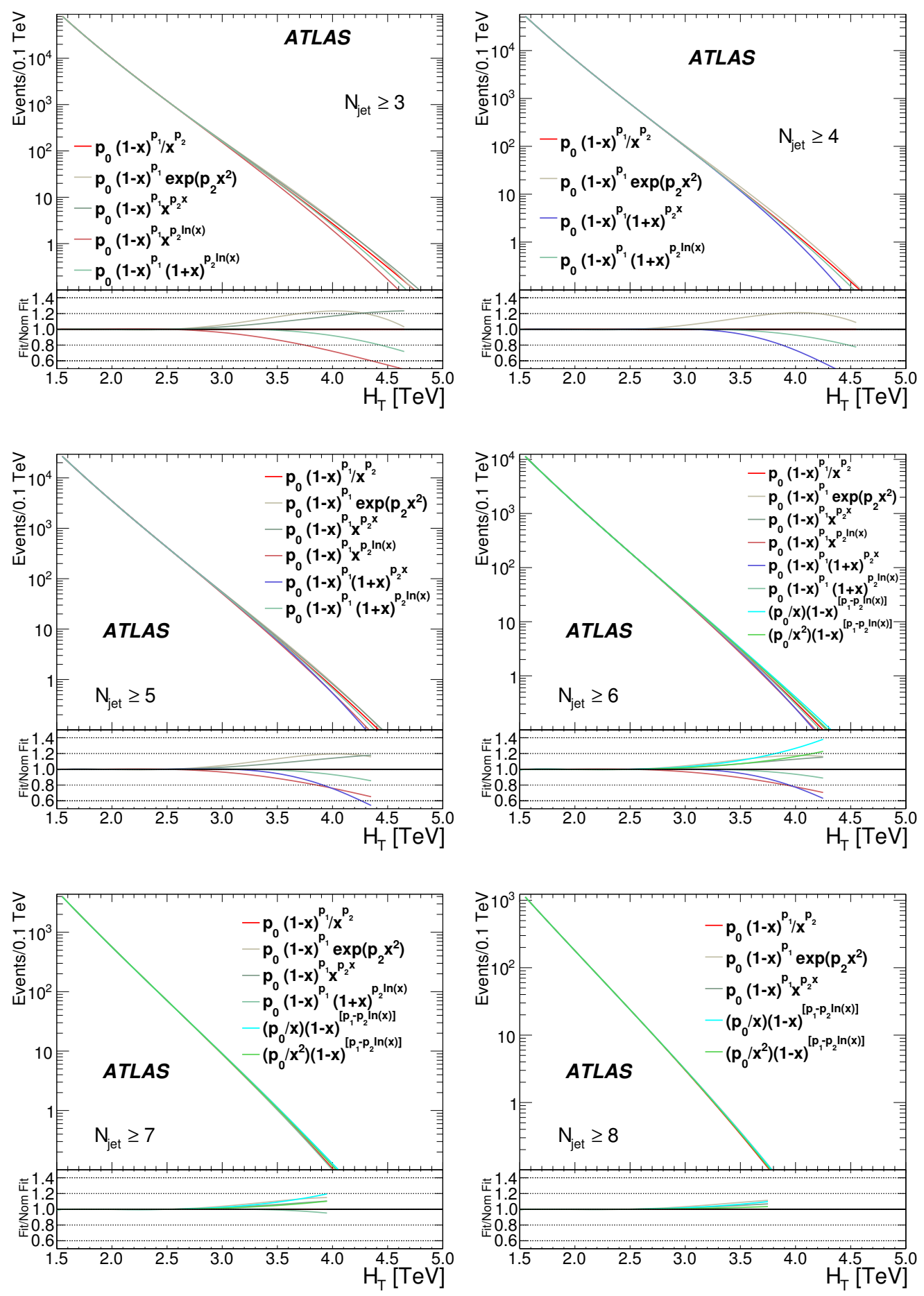

Figure 3. The $H_{\mathrm{T}}$ distributions, showing alternative fit functions for different inclusive jet multiplicities $N_{\text {jet }}$. The systematic uncertainty due to the choice of fit function versus exclusive $H_{\mathrm{T}}$ is given by the envelope of all the functions. 

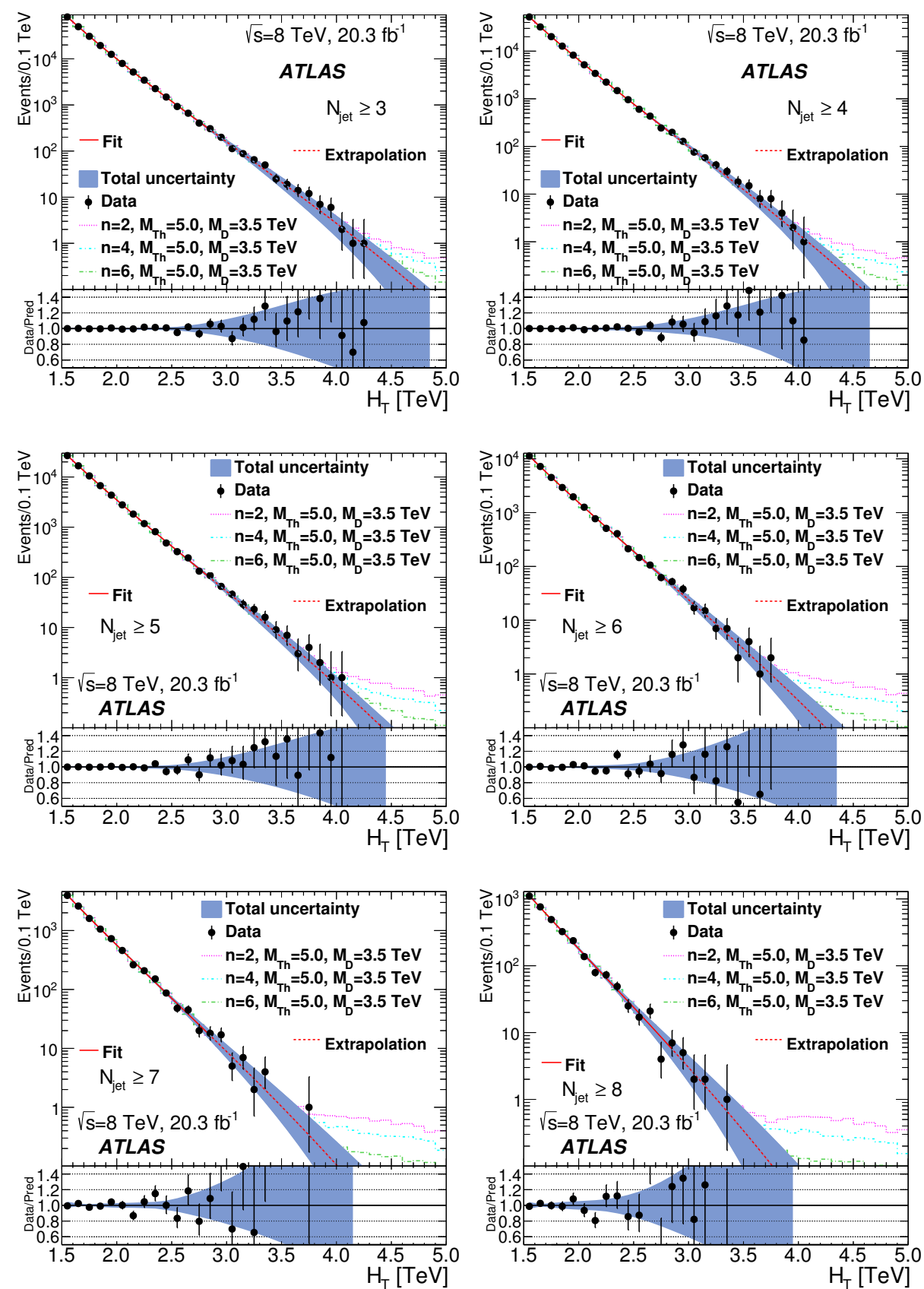

Figure 4. The $H_{\mathrm{T}}$ distributions, showing the data and extrapolated fits from the control region $1.5<H_{\mathrm{T}}<2.9 \mathrm{TeV}$ into the signal region $H_{\mathrm{T}}>3.0$ for each inclusive jet multiplicity $N_{\text {jet }}$. The uncertainty on the data points in both the distribution and ratio are due to the statistical uncertainty on the data only. The uncertainty band includes all uncertainties on the background prediction. Also shown are the expected black hole signals for three parameter sets of the CHARYBDIS2 nonrotating black hole model. 

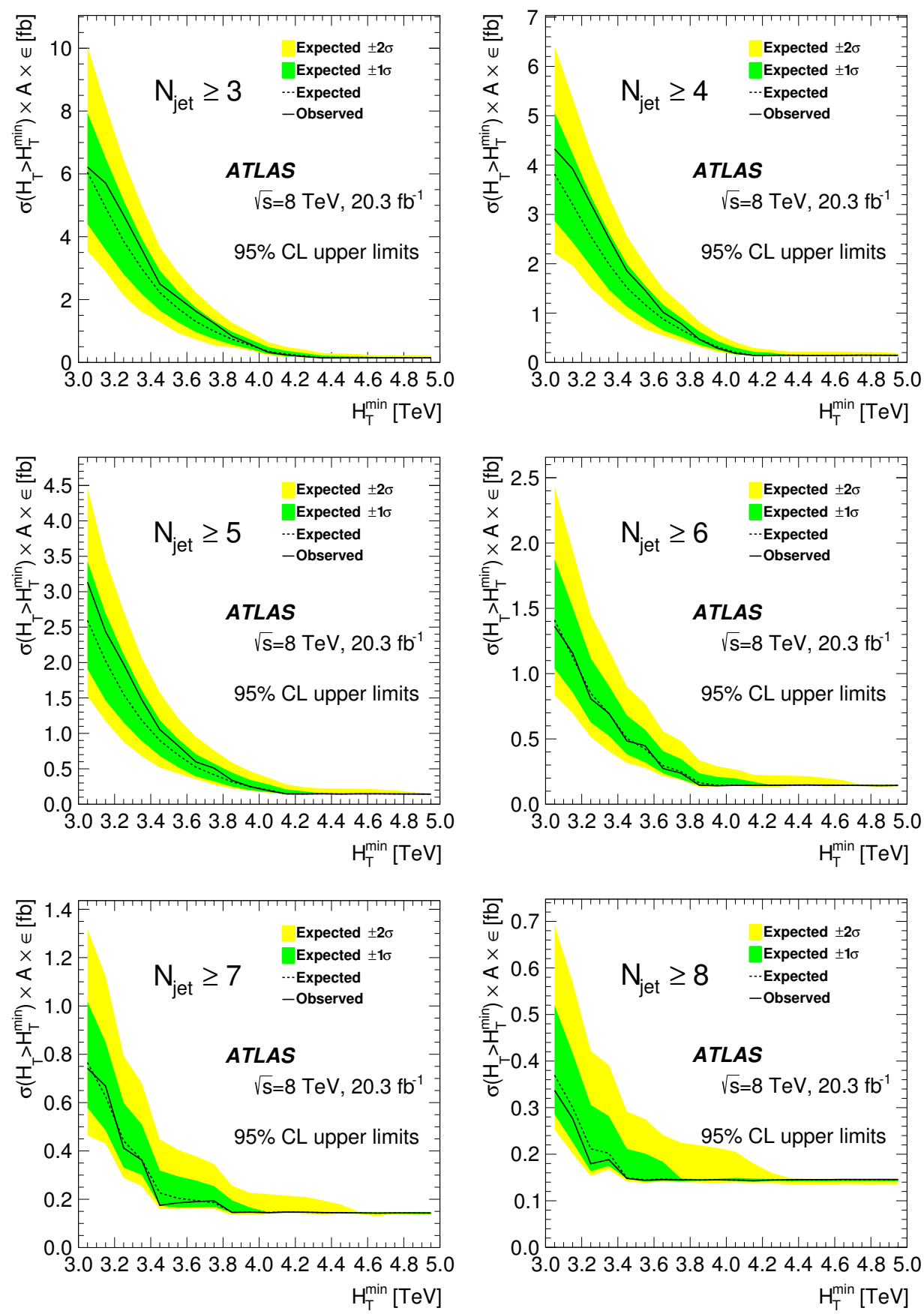

Figure 5. Upper limits on the visible cross section (cross section $(\sigma)$ times acceptance $(A)$ times efficiency $(\epsilon))$ at the $95 \%$ CL versus inclusive $H_{\mathrm{T}}$ for different inclusive jet multiplicities. The solid (dashed) lines correspond to the observed (expected) upper limits. The green (dark) and yellow (light) bands represent one and two standard deviations from the expected limits.

on several black hole and string ball signal models are set using the previous information, and estimates of the acceptance and efficiency for each model. The statistical proce- 
dure employed uses the one-sided profile-likelihood test statistic [44]. The uncertainties are modelled with a convolution of Gaussian probability density functions describing the uncertainties on the signal or on the background. The upper limits are derived from pseudo-experiments.

Counting experiments with $H_{\mathrm{T}}>H_{\mathrm{T}}^{\mathrm{min}}$ as a function of $H_{\mathrm{T}}^{\mathrm{min}}$ are performed for each inclusive jet multiplicity $N_{\text {jet }} \geq 3$ to $N_{\text {jet }} \geq 8$. Using the number of data events, estimated background, estimated uncertainty on the background, luminosity, and uncertainty on the luminosity, upper limits are calculated on the number of events divided by the integrated luminosity. Upper limits on the visible cross section (number of events divided by the luminosity or cross section times acceptance times reconstruction efficiency) at the 95\% CL are shown in figure 5 .

The expected uncertainty bands narrow at high $H_{\mathrm{T}}$ as the test statistic becomes discretely distributed due to the extremely small background prediction. These modelindependent limits on the cross section times acceptance times efficiency are as low as $0.14 \mathrm{fb}$ at the $95 \% \mathrm{CL}$ for minimum $H_{\mathrm{T}}$ values above $4.3 \mathrm{TeV}$ where no data events are observed.

To set limits on various models, the visible cross sections are divided by the reconstruction efficiencies to obtain limits on fiducial cross sections defined at the particle level. The reconstruction efficiencies are calculated by taking the total signal efficiency times acceptance and dividing by the fiducial acceptance. The fiducial acceptance at the generator particle level $^{2}$ is defined from the simulated signal events with final states that pass the jet $p_{\mathrm{T}}>50 \mathrm{GeV}$ and $|\eta|<2.8$ requirements. The events are then counted in the different inclusive $N_{\text {jet }}$ and $H_{\mathrm{T}}$ regions. Detector resolution can cause migration of signal events to different regions of $N_{\text {jet }}$ or $H_{\mathrm{T}}$. This can cause the reconstruction efficiency to be larger than unity in some $\left(N_{\text {jet }}, H_{\mathrm{T}}\right)$ regions.

The reconstruction efficiency depends on the particular black hole signal production model and kinematic region $\left(N_{\mathrm{jet}}, H_{\mathrm{T}}^{\mathrm{min}}\right)$ of interest. The efficiencies are determined over the range of $n, M_{D}$, and $M_{\text {th }}$ shown in figures 6-8. The efficiencies are practically independent of the number of extra dimensions in the model. The mean reconstruction efficiencies are about $88 \%$ with a variation between models of about $1 \%$. The RMS spread in efficiencies for a particular model is about $4 \%$ with a variation between models of about $1 \%$.

The limit on the cross section times acceptance is obtained from the visible cross section and the average reconstruction efficiency from many models, parameters, and kinematic properties. The limit on the cross section times acceptance is as low as $0.16 \mathrm{fb}$ at the 95\% CL for minimum $H_{\mathrm{T}}$ values above about $4.3 \mathrm{TeV}$. This is comparable to the results in ref. [17] in which missing transverse momentum is included in the definition of $H_{\mathrm{T}}$ if it is greater than $50 \mathrm{GeV}$. The results presented here are also compared to another ATLAS analysis [14], in which a high- $p_{\mathrm{T}}$ lepton was required. The intersection of these limits with theoretical predictions for the cross section within the fiducial selections used in this analysis could be used to constrain other models of new physics resulting in energetic

\footnotetext{
${ }^{2}$ This includes parton showering and jet clustering, using the anti- $k_{t}$ algorithm with $R=0.4$ on stable particles $(c \tau>10 \mathrm{~mm})$, including electrons and photons, but excluding muons and neutrinos.
} 

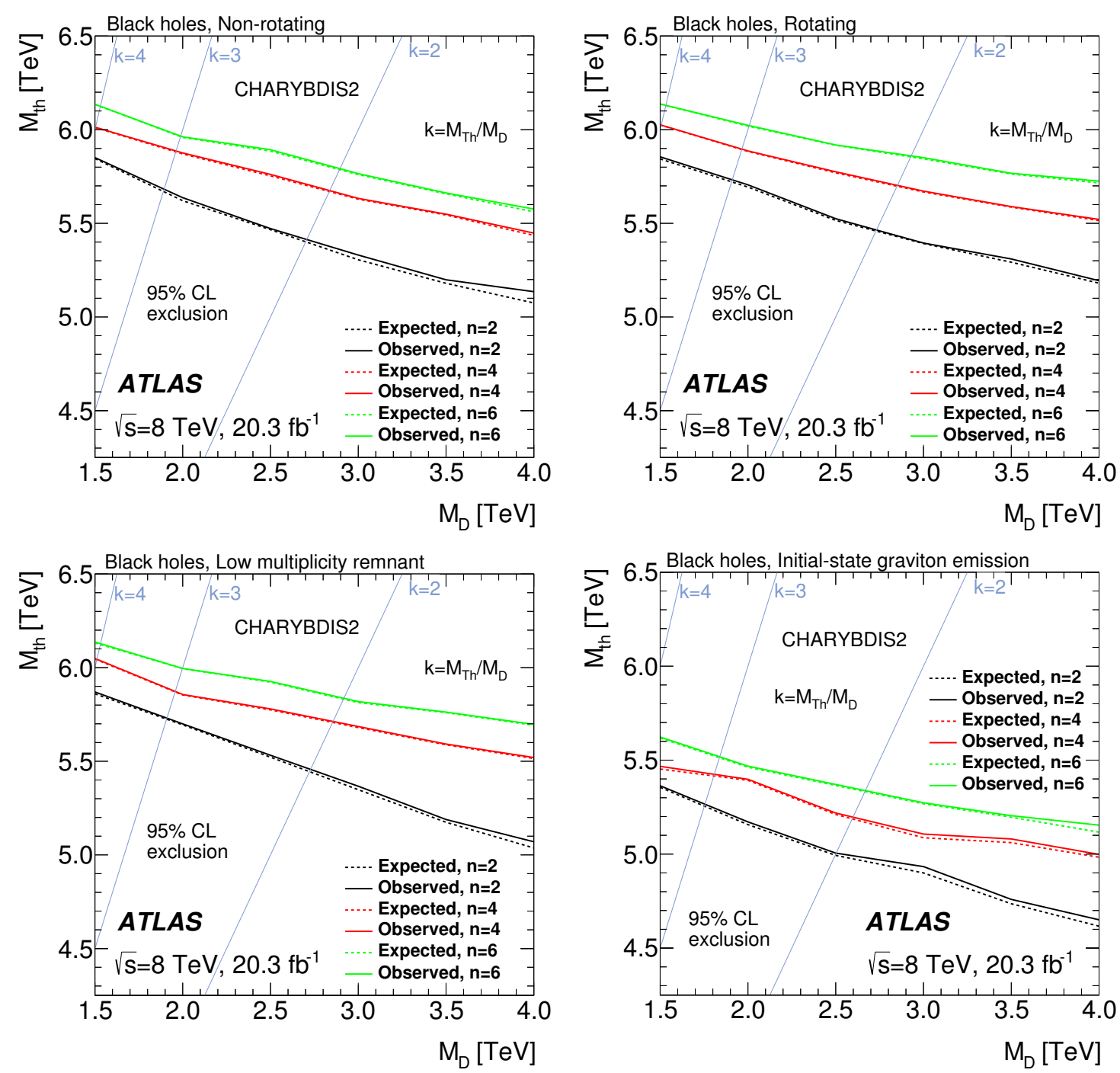

Figure 6. Exclusion contours in the $M_{\mathrm{th}}-M_{D}$ plane for different black hole models in two, four, and six extra dimensions simulated with CHARYBDIS2. The solid (dashed) lines show the observed (expected) 95\% CL limits. Masses below the corresponding lines are excluded. Lines of fixed $M_{\mathrm{th}} / M_{D}$ (defined as $k$ ) are shown. The assumptions of the models are valid for $k \gg 1$.

multi-jet final states.

Exclusion contours are obtained in the plane of $M_{D}$ and $M_{\mathrm{th}}$ for several models. For this purpose, counting experiments are performed to set a 95\% CL cross-section upper limit for each signal model and each $\left(M_{\mathrm{th}}, M_{D}\right)$ value of that signal model in this analysis. In setting these limits, both the estimated background uncertainty and signal uncertainties are taken into account. The background and its uncertainty are the same as described previously for the model-independent limits. The uncertainties on the signal include the uncertainty due to the jet energy scale and jet energy resolution, the statistical uncertainty on the signal MC samples, and the uncertainty on the integrated luminosity. As the 


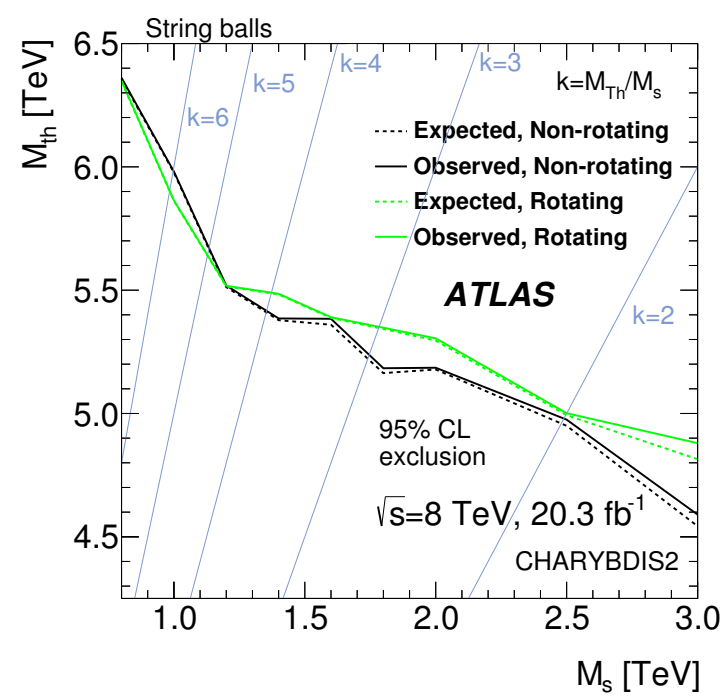

Figure 7. Exclusion contours in the $M_{\mathrm{th}}-M_{\mathrm{s}}$ plane for non-rotating and rotating string ball models simulated with CHARYBDIS2. The solid (dashed) lines show the observed (expected) 95\% CL limits. Masses below the corresponding lines are excluded. Lines of fixed $M_{\mathrm{th}} / M_{\mathrm{S}}$ (defined as $k$ ) are shown. The assumptions of the models are valid for $k \gg 1$.

cross section for black hole production is known only approximately and is highly model dependent, no theoretical uncertainty on the signal cross section is applied.

For each grid point in the $M_{\mathrm{th}}-M_{D}$ plane, the signal region which gives the lowest expected $p$-value is used. The most sensitive signal region for a particular signal model follows the kinematics of the signal model. For example, high-multiplicity signal regions are best for high-multiplicity signal samples, and high- $H_{\mathrm{T}}^{\min }$ regions are best for high- $M_{\mathrm{th}}$ signal samples. Observed and expected exclusion contours for different CHARYBDIS2 black hole models are shown in figure 6. The observed and expected exclusions for $n=2, n=4$, and $n=6$ are shown. In each exclusion figure, lines of fixed $M_{\mathrm{th}} / M_{D}$ (defined as $k$ ) are shown. The assumptions of the models are not valid for $k=1$, but are valid for $k \gg 1$. These lines therefore form useful guidelines as to the validity of the models across the plane. The results for non-rotating and rotating string balls are shown in figure 7 .

The exclusions tend to be stronger for higher $n$, due to the larger signal cross sections. For low values of $M_{\mathrm{th}} / M_{D}$, where there are the fewest Hawking emissions (and where the semi-classical production assumptions are least valid), the limits worsen. The exclusions for non-rotating and rotating black hole models, with all other parameters identical, appear similar. Including initial-state radiation reduces the cross section and hence the $\left(M_{\mathrm{th}}, M_{D}\right)$ exclusion reach. A low-multiplicity remnant state weakens the exclusion reach for $n=2$ at low values of $M_{\mathrm{th}} / M_{D}$, due to the reduced number of jets. For string balls, the exclusion for the rotating case is similar to the non-rotating case, in contrast to the result in ref. [14].

The exclusion contours for BlackMax models are shown in figure 8. They show the same general features as the ones obtained with samples generated by CHARYBDIS2. BlackMax 

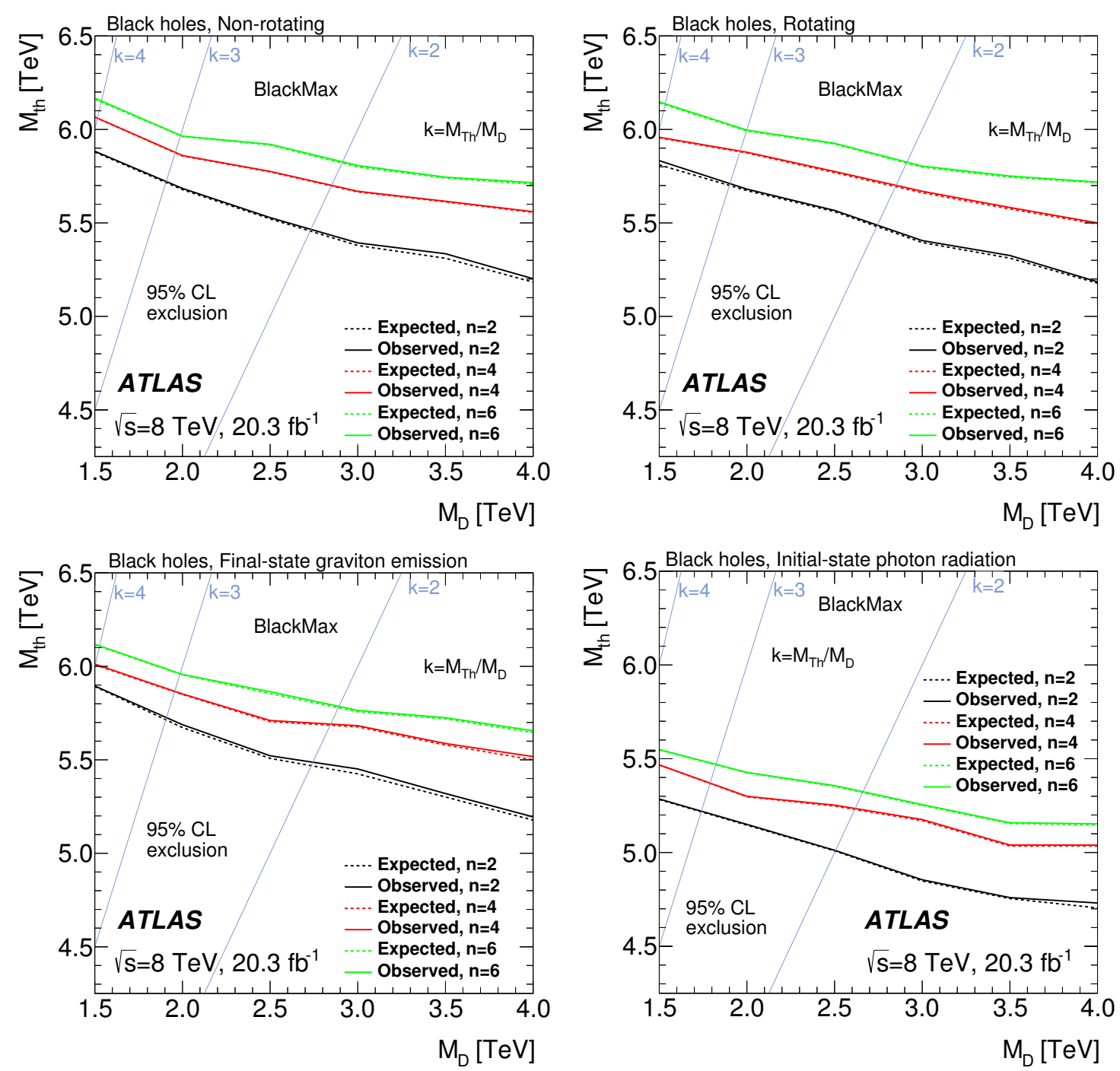

Figure 8. Exclusion contours in the $M_{\mathrm{th}}-M_{D}$ plane for different black hole models in two, four, and six extra dimensions simulated with BlackMax. The solid (dashed) lines show the observed (expected) 95\% CL limits. Masses below the corresponding lines are excluded. Lines of fixed $M_{\mathrm{th}} / M_{D}$ (defined as $k$ ) are shown. The assumptions of the models are valid for $k \gg 1$.

uses a final-burst remnant model, which gives high-multiplicity remnant states [33]. Comparing non-rotating and rotating CHARYBDIS2 and BlackMax results, shows this analysis is insensitive to the different remnant models. The results for the BlackMax model of production losses to photons is comparable to the results for the CHARYBDIS2 model of production losses to gravitons. Graviton emission in non-rotating black hole models weakens the exclusion slightly, as a greater number of decay products carry missing energy and do not contribute to the number of jets or $H_{\mathrm{T}}$.

Contour limits of $M_{\text {th }}$ versus $M_{D}$ are presented for a variety of models. These limits can be interpreted in terms of lower-mass limits on black hole and string ball masses that 
range from 4.6 to $6.2 \mathrm{TeV}$. This is again comparable to the results in ref. [17] with the limits here being about $0.1 \mathrm{TeV}$ higher in mass. The results presented here are also compared with those of ref. [14]. In the low- $M_{D}$ region the results are comparable, while in the high$M_{D}$ region the results presented here are a significant improvement over those in ref. [14]. The latter analysis is affected by a significant loss in sensitivity for the cases of rotating black holes and string balls, while the results presented here, and those in ref. [17], are rather independent of rotation.

\section{Conclusion}

The production of events with multiple high-transverse-momentum jets is measured using $20.3 \mathrm{fb}^{-1}$ of proton-proton collision data recorded at $\sqrt{s}=8 \mathrm{TeV}$ with the ATLAS detector at the LHC. No significant excess beyond SM expectations is observed, and upper limits on the visible cross sections for non-SM production of these final states are set. Using models for black hole and string ball production and decay, exclusion contours are determined as a function of mass threshold and the fundamental Planck scale.

The limit on the cross section times acceptance can be obtained from the visible cross section and the average reconstruction efficiency taken over many models, parameters, and kinematics. The limit on the cross section times acceptance is as low as $0.16 \mathrm{fb}$ at the 95\% CL for minimum $H_{\mathrm{T}}$ values above about $4.3 \mathrm{TeV}$.

Contour limits of $M_{\mathrm{th}}$ versus $M_{D}$ are presented for a variety of models. These limits can be interpreted in terms of lower-mass limits on black hole and string ball masses that range from 4.6 to $6.2 \mathrm{TeV}$.

\section{Acknowledgments}

We thank CERN for the very successful operation of the LHC, as well as the support staff from our institutions without whom ATLAS could not be operated efficiently.

We acknowledge the support of ANPCyT, Argentina; YerPhI, Armenia; ARC, Australia; BMWFW and FWF, Austria; ANAS, Azerbaijan; SSTC, Belarus; CNPq and FAPESP, Brazil; NSERC, NRC and CFI, Canada; CERN; CONICYT, Chile; CAS, MOST and NSFC, China; COLCIENCIAS, Colombia; MSMT CR, MPO CR and VSC CR, Czech Republic; DNRF, DNSRC and Lundbeck Foundation, Denmark; EPLANET, ERC and NSRF, European Union; IN2P3-CNRS, CEA-DSM/IRFU, France; GNSF, Georgia; BMBF, DFG, HGF, MPG and AvH Foundation, Germany; GSRT and NSRF, Greece; RGC, Hong Kong SAR, China; ISF, MINERVA, GIF, I-CORE and Benoziyo Center, Israel; INFN, Italy; MEXT and JSPS, Japan; CNRST, Morocco; FOM and NWO, Netherlands; BRF and RCN, Norway; MNiSW and NCN, Poland; GRICES and FCT, Portugal; MNE/IFA, Romania; MES of Russia and NRC KI, Russian Federation; JINR; MSTD, Serbia; MSSR, Slovakia; ARRS and MIZŠ, Slovenia; DST/NRF, South Africa; MINECO, Spain; SRC and Wallenberg Foundation, Sweden; SER, SNSF and Cantons of Bern and Geneva, Switzerland; NSC, Taiwan; TAEK, Turkey; STFC, the Royal Society and Leverhulme Trust, United Kingdom; DOE and NSF, United States of America. 
The crucial computing support from all WLCG partners is acknowledged gratefully, in particular from CERN and the ATLAS Tier-1 facilities at TRIUMF (Canada), NDGF (Denmark, Norway, Sweden), CC-IN2P3 (France), KIT/GridKA (Germany), INFN-CNAF (Italy), NL-T1 (Netherlands), PIC (Spain), ASGC (Taiwan), RAL (UK) and BNL (USA) and in the Tier-2 facilities worldwide.

Open Access. This article is distributed under the terms of the Creative Commons Attribution License (CC-BY 4.0), which permits any use, distribution and reproduction in any medium, provided the original author(s) and source are credited.

\section{References}

[1] N. Arkani-Hamed, S. Dimopoulos and G.R. Dvali, The Hierarchy problem and new dimensions at a millimeter, Phys. Lett. B 429 (1998) 263 [hep-ph/9803315] [INSPIRE].

[2] I. Antoniadis, N. Arkani-Hamed, S. Dimopoulos and G.R. Dvali, New dimensions at a millimeter to a Fermi and superstrings at a TeV, Phys. Lett. B 436 (1998) 257 [hep-ph/9804398] [INSPIRE].

[3] L. Randall and R. Sundrum, A Large mass hierarchy from a small extra dimension, Phys. Rev. Lett. 83 (1999) 3370 [hep-ph/9905221] [INSPIRE].

[4] L. Randall and R. Sundrum, An Alternative to compactification, Phys. Rev. Lett. 83 (1999) 4690 [hep-th/9906064] [INSPIRE].

[5] S. Dimopoulos and R. Emparan, String balls at the LHC and beyond, Phys. Lett. B 526 (2002) 393 [hep-ph/0108060] [INSPIRE].

[6] D.M. Gingrich, Experimental limits on the fundamental Planck scale in large extra dimensions, arXiv: 1210.5923 [INSPIRE].

[7] CDF collaboration, T. Aaltonen et al., Search for large extra dimensions in final states containing one photon or jet and large missing transverse energy produced in p $\bar{p}$ collisions at $\sqrt{s}=1.96-T e V$, Phys. Rev. Lett. 101 (2008) 181602 [arXiv:0807.3132] [INSPIRE].

[8] D0 collaboration, V.M. Abazov et al., Search for large extra dimensions via single photon plus missing energy final states at $\sqrt{s}=1.96-\mathrm{TeV}$, Phys. Rev. Lett. 101 (2008) 011601 [arXiv:0803.2137] [INSPIRE].

[9] ATLAS collaboration, Search for new phenomena in final states with an energetic jet and large missing transverse momentum in pp collisions at $\sqrt{s}=8 \mathrm{TeV}$ with the ATLAS detector, arXiv:1502.01518 [INSPIRE].

[10] CMS collaboration, Search for dark matter and large extra dimensions in monojet events in pp collisions at $\sqrt{s}=7 \mathrm{TeV}$, JHEP 09 (2012) 094 [arXiv:1206.5663] [INSPIRE].

[11] ATLAS collaboration, Search for strong gravity signatures in same-sign dimuon final states using the ATLAS detector at the LHC, Phys. Lett. B 709 (2012) 322 [arXiv:1111.0080] [INSPIRE].

[12] ATLAS collaboration, Search for TeV-scale gravity signatures in final states with leptons and jets with the ATLAS detector at $\sqrt{s}=7$ TeV, Phys. Lett. B 716 (2012) 122 [arXiv: 1204.4646] [INSPIRE]. 
[13] ATLAS collaboration, Search for microscopic black holes in a like-sign dimuon final state using large track multiplicity with the ATLAS detector, Phys. Rev. D 88 (2013) 072001 [arXiv: 1308.4075] [INSPIRE].

[14] ATLAS collaboration, Search for microscopic black holes and string balls in final states with leptons and jets with the ATLAS detector at $\sqrt{s}=8$ TeV, JHEP 08 (2014) 103 [arXiv: 1405.4254] [INSPIRE].

[15] CMS collaboration, Search for Microscopic Black Hole Signatures at the Large Hadron Collider, Phys. Lett. B 697 (2011) 434 [arXiv:1012.3375] [inSPIRE].

[16] CMS collaboration, Search for microscopic black holes in pp collisions at $\sqrt{s}=7$ TeV, JHEP 04 (2012) 061 [arXiv: 1202.6396] [INSPIRE].

[17] CMS collaboration, Search for microscopic black holes in pp collisions at $\sqrt{s}=8 \mathrm{TeV}$, JHEP 07 (2013) 178 [arXiv: 1303.5338] [INSPIRE].

[18] S. Dimopoulos and G.L. Landsberg, Black holes at the LHC, Phys. Rev. Lett. 87 (2001) 161602 [hep-ph/0106295] [INSPIRE].

[19] S.B. Giddings and S.D. Thomas, High-energy colliders as black hole factories: The End of short distance physics, Phys. Rev. D 65 (2002) 056010 [hep-ph/0106219] [INSPIRE].

[20] S.W. Hawking, Particle Creation by Black Holes, Commun. Math. Phys. 43 (1975) 199 [INSPIRE].

[21] D.M. Gingrich and K. Martell, Study of highly-excited string states at the Large Hadron Collider, Phys. Rev. D 78 (2008) 115009 [arXiv:0808.2512] [inSPIRE].

[22] ATLAS collaboration, The ATLAS experiment at the CERN Large Hadron Collider, 2008 JINST 3 S08003.

[23] ATLAS collaboration, Performance of the ATLAS Trigger System in 2010, Eur. Phys. J. C 72 (2012) 1849 [arXiv:1110.1530] [INSPIRE].

[24] T. Sjöstrand, S. Mrenna and P.Z. Skands, A Brief Introduction to PYTHIA 8.1, Comput. Phys. Commun. 178 (2008) 852 [arXiv:0710.3820] [INSPIRE].

[25] ATLAS collaboration, Summary of ATLAS PYTHIA 8 tunes, ATL-PHYS-PUB-2012-003 (2012).

[26] H.-L. Lai et al., New parton distributions for collider physics, Phys. Rev. D 82 (2010) 074024 [arXiv: 1007.2241] [INSPIRE].

[27] M. Bähr et al., HERWIG++ Physics and Manual, Eur. Phys. J. C 58 (2008) 639 [arXiv: 0803.0883] [INSPIRE].

[28] J. Pumplin et al., New generation of parton distributions with uncertainties from global QCD analysis, JHEP 07 (2002) 012 [hep-ph/0201195] [INSPIRE].

[29] M.L. Mangano, M. Moretti, F. Piccinini, R. Pittau and A.D. Polosa, ALPGEN, a generator for hard multiparton processes in hadronic collisions, JHEP 07 (2003) 001 [hep-ph/0206293] [INSPIRE].

[30] T. Gleisberg et al., Event generation with SHERPA 1.1, JHEP 02 (2009) 007 [arXiv:0811.4622] [INSPIRE].

[31] S. Agostinelli et al., Geant4 - a simulation toolkit, Nucl. Instrum. Meth. A 506 (2003) 250. 
[32] J.A. Frost et al., Phenomenology of Production and Decay of Spinning Extra-Dimensional Black Holes at Hadron Colliders, JHEP 10 (2009) 014 [arXiv:0904.0979] [INSPIRE].

[33] D.-C. Dai et al., BlackMax: A black-hole event generator with rotation, recoil, split branes and brane tension, Phys. Rev. D 77 (2008) 076007 [arXiv: 0711.3012] [INSPIRE].

[34] A.D. Martin, W.J. Stirling, R.S. Thorne and G. Watt, Parton distributions for the LHC, Eur. Phys. J. C 63 (2009) 189 [arXiv:0901.0002] [inSPIRE].

[35] ATLAS collaboration, The simulation principle and performance of the ATLAS fast calorimeter simulation FastCaloSim, ATL-PHYS-PUB-2010-013 (2010).

[36] ATLAS collaboration, The ATLAS Simulation Infrastructure, Eur. Phys. J. C 70 (2010) 823 [arXiv: 1005.4568] [INSPIRE].

[37] ATLAS collaboration, Improved luminosity determination in pp collisions at $\sqrt{s}=7 \mathrm{TeV}$ using the ATLAS detector at the LHC, Eur. Phys. J. C 73 (2013) 2518 [arXiv:1302.4393] [INSPIRE].

[38] M. Cacciari, G.P. Salam and G. Soyez, The Anti-k(t) jet clustering algorithm, JHEP 04 (2008) 063 [arXiv:0802.1189] [INSPIRE].

[39] W. Lampl et al., Calorimeter Clustering Algorithms : Description and Performance, ATL-LARG-PUB-2008-002 (2008).

[40] ATLAS collaboration, Jet energy measurement and its systematic uncertainty in proton-proton collisions at $\sqrt{s}=7 \mathrm{TeV}$ with the ATLAS detector, Eur. Phys. J. C 75 (2015) 17 [arXiv: 1406.0076] [INSPIRE].

[41] ATLAS collaboration, Search for new phenomena in the dijet mass distribution using $p-p$ collision data at $\sqrt{s}=8 \mathrm{TeV}$ with the ATLAS detector, Phys. Rev. D 91 (2015) 052007 [arXiv: 1407.1376] [INSPIRE].

[42] G. Choudalakis, On hypothesis testing, trials factor, hypertests and the BumpHunter, arXiv: 1101.0390 [INSPIRE].

[43] G. Choudalakis and D. Casadei, Plotting the differences between data and expectation, Eur. Phys. J. Plus 127 (2012) 25 [arXiv:1111.2062].

[44] G. Cowan, K. Cranmer, E. Gross and O. Vitells, Asymptotic formulae for likelihood-based tests of new physics, Eur. Phys. J. C 71 (2011) 1554 [arXiv:1007.1727] [INSPIRE]. 


\section{The ATLAS collaboration}

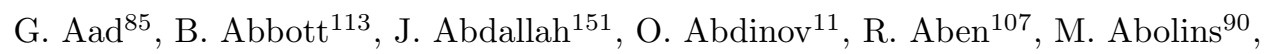
O.S. AbouZeid ${ }^{158}$, H. Abramowicz ${ }^{153}$, H. Abreu ${ }^{152}$, R. Abreu ${ }^{30}$, Y. Abulaiti ${ }^{146 a, 146 b}$, B.S. Acharya ${ }^{164 a, 164 b, a}$, L. Adamczyk ${ }^{38 a}$, D.L. Adams ${ }^{25}$, J. Adelman ${ }^{108}$, S. Adomeit ${ }^{100}$, T. Adye ${ }^{131}$, A.A. Affolder ${ }^{74}$, T. Agatonovic-Jovin ${ }^{13}$, J.A. Aguilar-Saavedra ${ }^{126 a, 126 f}$, S.P. Ahlen ${ }^{22}$, F. Ahmadov ${ }^{65, b}$, G. Aielli133a,133b ${ }^{13}$, H. Akerstedt ${ }^{146 a, 146 b}$, T.P.A. Åkesson ${ }^{81}$, G. Akimoto ${ }^{155}$, A.V. Akimov ${ }^{96}$, G.L. Alberghi ${ }^{20 a}{ }^{20 b}$, J. Albert ${ }^{169}$, S. Albrand ${ }^{55}$, M.J. Alconada Verzini ${ }^{71}$, M. Aleksa ${ }^{30}$, I.N. Aleksandrov ${ }^{65}$, C. Alexa ${ }^{26 a}$, G. Alexander ${ }^{153}$, T. Alexopoulos ${ }^{10}$, M. Alhroob $^{113}$, G. Alimonti ${ }^{91 a}$, L. Alio ${ }^{85}$, J. Alison ${ }^{31}$, S.P. Alkire ${ }^{35}$, B.M.M. Allbrooke ${ }^{18}$, P.P. Allport ${ }^{74}$,

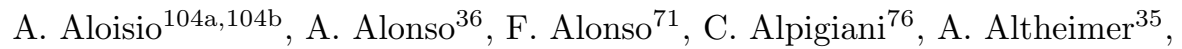

B. Alvarez Gonzalez ${ }^{30}$, D. Álvarez Piqueras ${ }^{167}$, M.G. Alviggi ${ }^{104 a, 104 b}$, B.T. Amadio ${ }^{15}$,

K. Amako ${ }^{66}$, Y. Amaral Coutinho ${ }^{24 a}$, C. Amelung ${ }^{23}$, D. Amidei ${ }^{89}$, S.P. Amor Dos Santos ${ }^{126 a, 126 c}$, A. Amorim ${ }^{126 a, 126 b}$, S. Amoroso ${ }^{48}$, N. Amram ${ }^{153}$, G. Amundsen ${ }^{23}$, C. Anastopoulos ${ }^{139}$, L.S. Ancu ${ }^{49}$, N. Andari ${ }^{30}$, T. Andeen ${ }^{35}$, C.F. Anders ${ }^{58 b}$, G. Anders ${ }^{30}$, J.K. Anders ${ }^{74}$, K.J. Anderson ${ }^{31}$, A. Andreazza ${ }^{91 a, 91 b}$, V. Andrei ${ }^{58 a}$, S. Angelidakis ${ }^{9}$, I. Angelozzi ${ }^{107}$, P. Anger ${ }^{44}$, A. Angerami ${ }^{35}$, F. Anghinolfi ${ }^{30}$, A.V. Anisenkov ${ }^{109, c}$, N. Anjos ${ }^{12}$, A. Annovi ${ }^{124 a, 124 b}$, M. Antonelli ${ }^{47}$, A. Antonov ${ }^{98}$, J. Antos ${ }^{144 b}$, F. Anulli ${ }^{132 a}$, M. Aoki ${ }^{66}$, L. Aperio Bella ${ }^{18}$, G. Arabidze ${ }^{90}$, Y. Arai ${ }^{66}$, J.P. Araque ${ }^{126 a}$, A.T.H. Arce ${ }^{45}$, F.A. Arduh ${ }^{71}$, J-F. Arguin ${ }^{95}$, S. Argyropoulos ${ }^{42}$, M. Arik ${ }^{19 a}$, A.J. Armbruster ${ }^{30}$, O. Arnaez ${ }^{30}$, V. Arnal ${ }^{82}$, H. Arnold ${ }^{48}$, M. Arratia ${ }^{28}$, O. Arslan ${ }^{21}$, A. Artamonov ${ }^{97}$, G. Artoni ${ }^{23}$, S. Asai ${ }^{155}$, N. Asbah ${ }^{42}$, A. Ashkenazi ${ }^{153}$, B. Åsman 146a,146b, L. Asquith ${ }^{149}$, K. Assamagan ${ }^{25}$, R. Astalos ${ }^{144 a}$, M. Atkinson ${ }^{165}$, N.B. Atlay ${ }^{141}$, B. Auerbach ${ }^{6}$, K. Augsten ${ }^{128}$, M. Aurousseau ${ }^{145 b}$, G. Avolio ${ }^{30}$, B. Axen ${ }^{15}$, M.K. Ayoub ${ }^{117}$, G. Azuelos ${ }^{95, d}$, M.A. Baak ${ }^{30}$, A.E. Baas ${ }^{58 a}$, C. Bacci ${ }^{134 a, 134 b}$, H. Bachacou ${ }^{136}$, K. Bachas ${ }^{154}$, M. Backes ${ }^{30}$, M. Backhaus ${ }^{30}$, P. Bagiacchi132a,132b, P. Bagnaia ${ }^{132 a, 132 b}$, Y. Bai ${ }^{33 a}$, T. Bain ${ }^{35}$, J.T. Baines ${ }^{131}$, O.K. Baker ${ }^{176}$, P. Balek ${ }^{129}$, T. Balestri ${ }^{148}$, F. Balli ${ }^{84}$, E. Banas ${ }^{39}$, Sw. Banerjee ${ }^{173}$, A.A.E. Bannoura ${ }^{175}$, H.S. Bansil ${ }^{18}$, L. Barak ${ }^{30}$, E.L. Barberio ${ }^{88}$, D. Barberis ${ }^{50 a, 50 b}$, M. Barbero ${ }^{85}$, T. Barillari ${ }^{101}$, M. Barisonzi ${ }^{164 a, 164 b}$, T. Barklow ${ }^{143}$, N. Barlow ${ }^{28}$, S.L. Barnes ${ }^{84}$, B.M. Barnett ${ }^{131}$, R.M. Barnett ${ }^{15}$, Z. Barnovska ${ }^{5}$, A. Baroncelli ${ }^{134 a}$, G. Barone ${ }^{49}$, A.J. Barr ${ }^{120}$, F. Barreiro ${ }^{82}$, J. Barreiro Guimarães da Costa ${ }^{57}$, R. Bartoldus ${ }^{143}$, A.E. Barton ${ }^{72}$, P. Bartos ${ }^{144 a}$, A. Basalaev ${ }^{123}$, A. Bassalat ${ }^{117}$, A. Basye ${ }^{165}$, R.L. Bates ${ }^{53}$, S.J. Batista ${ }^{158}$, J.R. Batley ${ }^{28}$, M. Battaglia ${ }^{137}$, M. Bauce ${ }^{132 a, 132 b}$, F. Bauer ${ }^{136}$, H.S. Bawa ${ }^{143, e}$, J.B. Beacham ${ }^{111}$, M.D. Beattie ${ }^{72}$, T. Beau ${ }^{80}$, P.H. Beauchemin ${ }^{161}$, R. Beccherle ${ }^{124 a, 124 b}$, P. Bechtle ${ }^{21}$, H.P. Beck ${ }^{17, f}$, K. Becker ${ }^{120}$, M. Becker ${ }^{83}$, S. Becker ${ }^{100}$, M. Beckingham ${ }^{170}$, C. Becot ${ }^{117}$, A.J. Beddall ${ }^{19 c}$, A. Beddall ${ }^{19 c}$, V.A. Bednyakov ${ }^{65}$, C.P. Bee ${ }^{148}$, L.J. Beemster ${ }^{107}$, T.A. Beermann ${ }^{175}$, M. Begel ${ }^{25}$, J.K. Behr ${ }^{120}$, C. Belanger-Champagne ${ }^{87}$, W.H. Bell ${ }^{49}$, G. Bella ${ }^{153}$, L. Bellagamba ${ }^{20 a}$, A. Bellerive ${ }^{29}$, M. Bellomo ${ }^{86}$, K. Belotskiy ${ }^{98}$, O. Beltramello ${ }^{30}$, O. Benary ${ }^{153}$, D. Benchekroun ${ }^{135 a}$, M. Bender ${ }^{100}$, K. Bendtz ${ }^{146 a, 146 b}$, N. Benekos ${ }^{10}$, Y. Benhammou ${ }^{153}$, E. Benhar Noccioli ${ }^{49}$,

J.A. Benitez Garcia ${ }^{159 b}$, D.P. Benjamin ${ }^{45}$, J.R. Bensinger ${ }^{23}$, S. Bentvelsen ${ }^{107}$, L. Beresford ${ }^{120}$, M. Beretta ${ }^{47}$, D. Berge ${ }^{107}$, E. Bergeaas Kuutmann ${ }^{166}$, N. Berger ${ }^{5}$, F. Berghaus ${ }^{169}$, J. Beringer ${ }^{15}$, C. Bernard ${ }^{22}$, N.R. Bernard ${ }^{86}$, C. Bernius ${ }^{110}$, F.U. Bernlochner ${ }^{21}$, T. Berry ${ }^{77}$, P. Berta ${ }^{129}$, C. Bertella ${ }^{83}$, G. Bertoli ${ }^{146 a, 146 b}$, F. Bertolucci ${ }^{124 a, 124 b}$, C. Bertsche ${ }^{113}$, D. Bertsche ${ }^{113}$, M.I. Besana ${ }^{91 a}$, G.J. Besjes ${ }^{106}$, O. Bessidskaia Bylund ${ }^{146 a, 146 b}$, M. Bessner ${ }^{42}$, N. Besson ${ }^{136}$, C. Betancourt ${ }^{48}$, S. Bethke ${ }^{101}$, A.J. Bevan ${ }^{76}$, W. Bhimji ${ }^{46}$, R.M. Bianchi ${ }^{125}$, L. Bianchini2 ${ }^{23}$, M. Bianco ${ }^{30}$, O. Biebel ${ }^{100}$, S.P. Bieniek ${ }^{78}$, M. Biglietti ${ }^{134 a}$, J. Bilbao De Mendizabal ${ }^{49}$, H. Bilokon ${ }^{47}$, M. Bindi ${ }^{54}$, S. Binet ${ }^{117}$, A. Bingul ${ }^{19 c}$, C. Bini ${ }^{132 a, 132 b}$, C.W. Black ${ }^{150}$, J.E. Black ${ }^{143}$, K.M. Black ${ }^{22}$, D. Blackburn ${ }^{138}$, R.E. Blair ${ }^{6}$, J.-B. Blanchard ${ }^{136}$, J.E. Blanco ${ }^{77}$, T. Blazek ${ }^{144 a}$, I. Bloch ${ }^{42}$, C. Blocker ${ }^{23}$, W. Blum ${ }^{83, *}$, U. Blumenschein ${ }^{54}$, G.J. Bobbink ${ }^{107}$, 
V.S. Bobrovnikov ${ }^{109, c}$, S.S. Bocchetta ${ }^{81}$, A. Bocci ${ }^{45}$, C. Bock ${ }^{100}$, M. Boehler ${ }^{48}$, J.A. Bogaerts ${ }^{30}$, A.G. Bogdanchikov ${ }^{109}$, C. Bohm ${ }^{146 a}$, V. Boisvert ${ }^{77}$, T. Bold ${ }^{38 a}$, V. Boldea ${ }^{26 a}$, A.S. Boldyrev ${ }^{99}$, M. Bomben ${ }^{80}$, M. Bona ${ }^{76}$, M. Boonekamp ${ }^{136}$, A. Borisov ${ }^{130}$, G. Borissov ${ }^{72}$, S. Borroni ${ }^{42}$, J. Bortfeldt ${ }^{100}$, V. Bortolotto ${ }^{60 a, 60 b, 60 c}$, K. Bos ${ }^{107}$, D. Boscherini ${ }^{20 a}$, M. Bosman ${ }^{12}$, J. Boudreau ${ }^{125}$, J. Bouffard ${ }^{2}$, E.V. Bouhova-Thacker ${ }^{72}$, D. Boumediene ${ }^{34}$, C. Bourdarios ${ }^{117}$, N. Bousson ${ }^{114}$, A. Boveia ${ }^{30}$, J. Boyd ${ }^{30}$, I.R. Boyko ${ }^{65}$, I. Bozic ${ }^{13}$, J. Bracinik $^{18}$, A. Brandt ${ }^{8}$, G. Brandt ${ }^{54}$, O. Brandt ${ }^{58 a}$, U. Bratzler ${ }^{156}$, B. Brau ${ }^{86}$, J.E. Brau ${ }^{116}$, H.M. Braun ${ }^{175, *}$, S.F. Brazzale ${ }^{164 a, 164 c}$, K. Brendlinger ${ }^{122}$, A.J. Brennan ${ }^{88}$, L. Brenner ${ }^{107}$, R. Brenner ${ }^{166}$, S. Bressler ${ }^{172}$, K. Bristow ${ }^{145 c}$, T.M. Bristow ${ }^{46}$, D. Britton ${ }^{53}$, D. Britzger ${ }^{42}$, F.M. Brochu ${ }^{28}$, I. Brock ${ }^{21}$, R. Brock ${ }^{90}$, J. Bronner ${ }^{101}$, G. Brooijmans ${ }^{35}$, T. Brooks ${ }^{77}$, W.K. Brooks ${ }^{32 b}$, J. Brosamer ${ }^{15}$, E. Brost ${ }^{116}$, J. Brown ${ }^{55}$, P.A. Bruckman de Renstrom ${ }^{39}$, D. Bruncko ${ }^{144 \mathrm{~b}}$, R. Bruneliere ${ }^{48}$, A. Bruni ${ }^{20 a}$, G. Bruni ${ }^{20 a}$, M. Bruschi ${ }^{20 a}$, L. Bryngemark $^{81}$, T. Buanes ${ }^{14}$, Q. Buat ${ }^{142}$, P. Buchholz ${ }^{141}$, A.G. Buckley ${ }^{53}$, S.I. Buda ${ }^{26 a}$, I.A. Budagov ${ }^{65}$, F. Buehrer ${ }^{48}$, L. Bugge ${ }^{119}$, M.K. Bugge ${ }^{119}$, O. Bulekov ${ }^{98}$, D. Bullock ${ }^{8}$, H. Burckhart ${ }^{30}$, S. Burdin ${ }^{74}$, B. Burghgrave ${ }^{108}$, S. Burke ${ }^{131}$, I. Burmeister ${ }^{43}$, E. Busato ${ }^{34}$, D. Büscher ${ }^{48}$, V. Büscher ${ }^{83}$, P. Bussey ${ }^{53}$, J.M. Butler ${ }^{22}$, A.I. Butt ${ }^{3}$, C.M. Buttar ${ }^{53}$, J.M. Butterworth ${ }^{78}$, P. Butti ${ }^{107}$, W. Buttinger ${ }^{25}$, A. Buzatu ${ }^{53}$, A.R. Buzykaev ${ }^{109, c}$, S. Cabrera Urbán ${ }^{167}$, D. Caforio ${ }^{128}$, V.M. Cairo ${ }^{37 a, 37 b}$, O. Cakir ${ }^{4 a}$, P. Calafiura ${ }^{15}$, A. Calandri ${ }^{136}$, G. Calderini ${ }^{80}$, P. Calfayan ${ }^{100}$, L.P. Caloba ${ }^{24 a}$, D. Calvet $^{34}$, S. Calvet ${ }^{34}$, R. Camacho Toro ${ }^{49}$, S. Camarda ${ }^{42}$, P. Camarri ${ }^{133 a, 133 b}$, D. Cameron ${ }^{119}$, L.M. Caminada ${ }^{15}$, R. Caminal Armadans ${ }^{12}$, S. Campana ${ }^{30}$, M. Campanelli ${ }^{78}$, A. Campoverde ${ }^{148}$, V. Canale ${ }^{104 a, 104 b}$, A. Canepa ${ }^{159 a}$, M. Cano Bret ${ }^{76}$, J. Cantero ${ }^{82}$, R. Cantrill ${ }^{126 a}$, T. $\mathrm{Cao}^{40}$, M.D.M. Capeans Garrido ${ }^{30}$, I. Caprini ${ }^{26 a}$, M. Caprini ${ }^{26 a}$, M. Capua ${ }^{37 a, 37 b}$, R. Caputo $^{83}$, R. Cardarelli ${ }^{133 a}$, T. Carli ${ }^{30}$, G. Carlino ${ }^{104 a}$, L. Carminati91a,91b, S. Caron ${ }^{106}$, E. Carquin ${ }^{32 a}$, G.D. Carrillo-Montoya ${ }^{8}$, J.R. Carter ${ }^{28}$, J. Carvalho ${ }^{126 a, 126 c}$, D. Casadei $^{78}$, M.P. Casado ${ }^{12}$, M. Casolino ${ }^{12}$, E. Castaneda-Miranda ${ }^{145 b}$, A. Castelli ${ }^{107}$, V. Castillo Gimenez ${ }^{167}$, N.F. Castro ${ }^{126 a, g}$, P. Catastini ${ }^{57}$, A. Catinaccio ${ }^{30}$, J.R. Catmore ${ }^{119}$, A. Cattai ${ }^{30}$, J. Caudron ${ }^{83}$, V. Cavaliere ${ }^{165}$, D. Cavalli ${ }^{91 a}$, M. Cavalli-Sforza ${ }^{12}$, V. Cavasinni ${ }^{124 a, 124 b}$, F. Ceradini ${ }^{134 a, 134 b}$, B.C. Cerio ${ }^{45}$, K. Cerny ${ }^{129}$, A.S. Cerqueira ${ }^{24 b}$,

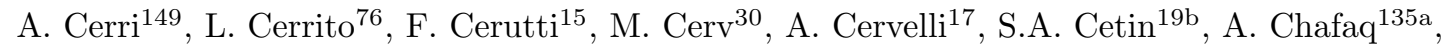
D. Chakraborty ${ }^{108}$, I. Chalupkova ${ }^{129}$, P. Chang ${ }^{165}$, B. Chapleau ${ }^{87}$, J.D. Chapman ${ }^{28}$, D.G. Charlton ${ }^{18}$, C.C. Chau ${ }^{158}$, C.A. Chavez Barajas ${ }^{149}$, S. Cheatham ${ }^{152}$, A. Chegwidden ${ }^{90}$, S. Chekanov ${ }^{6}$, S.V. Chekulaev ${ }^{159 a}$, G.A. Chelkov ${ }^{65, h}$, M.A. Chelstowska ${ }^{89}$, C. Chen ${ }^{64}$, H. Chen ${ }^{25}$, K. Chen ${ }^{148}$, L. Chen ${ }^{33 d, i}$, S. Chen ${ }^{33 c}$, X. Chen ${ }^{33 f}$, Y. Chen ${ }^{67}$, H.C. Cheng ${ }^{89}$, Y. Cheng ${ }^{31}$,

A. Cheplakov ${ }^{65}$, E. Cheremushkina ${ }^{130}$, R. Cherkaoui El Moursli ${ }^{135 e}$, V. Chernyatin ${ }^{25, *}$, E. Cheu $^{7}$, L. Chevalier ${ }^{136}$, V. Chiarella ${ }^{47}$, J.T. Childers ${ }^{6}$, G. Chiodini ${ }^{73 a}$, A.S. Chisholm ${ }^{18}$, R.T. Chislett $^{78}$, A. Chitan ${ }^{26 a}$, M.V. Chizhov ${ }^{65}$, K. Choi ${ }^{61}$, S. Chouridou ${ }^{9}$, B.K.B. Chow ${ }^{100}$, V. Christodoulou ${ }^{78}$, D. Chromek-Burckhart ${ }^{30}$, M.L. $\mathrm{Chu}^{151}$, J. Chudoba ${ }^{127}$, A.J. Chuinard ${ }^{87}$, J.J. Chwastowski ${ }^{39}$, L. Chytka ${ }^{115}$, G. Ciapetti132a,132b , A.K. Ciftci ${ }^{4 a}$, D. Cinca ${ }^{53}$, V. Cindro ${ }^{75}$, I.A. Cioara ${ }^{21}$, A. Ciocio $^{15}$, Z.H. Citron ${ }^{172}$, M. Ciubancan ${ }^{26 a}$, A. Clark $^{49}$, B.L. Clark ${ }^{57}$, P.J. Clark ${ }^{46}$, R.N. Clarke ${ }^{15}$, W. Cleland ${ }^{125}$, C. Clement ${ }^{146 a, 146 b}$, Y. Coadou ${ }^{85}$, M. Cobal ${ }^{164 a, 164 c}$, A. Coccaro ${ }^{138}$, J. Cochran ${ }^{64}$, L. Coffey ${ }^{23}$, J.G. Cogan ${ }^{143}$, B. Cole ${ }^{35}$, S. Cole ${ }^{108}$, A.P. Colijn ${ }^{107}$, J. Collot ${ }^{55}$, T. Colombo ${ }^{58 c}$, G. Compostella ${ }^{101}$, P. Conde Muiño ${ }^{126 a, 126 b}$, E. Coniavitis ${ }^{48}$, S.H. Connell ${ }^{145 b}$, I.A. Connelly ${ }^{77}$, S.M. Consonni ${ }^{91 a, 91 b}$, V. Consorti ${ }^{48}$, S. Constantinescu ${ }^{26 a}$, C. Conta ${ }^{121 a, 121 b}$, G. Conti ${ }^{30}$, F. Conventi1 ${ }^{104 a}, j$, M. Cooke ${ }^{15}$, B.D. Cooper ${ }^{78}$, A.M. Cooper-Sarkar ${ }^{120}$, T. Cornelissen ${ }^{175}$, M. Corradi ${ }^{20 a}$, F. Corriveau ${ }^{87, k}$, A. Corso-Radu ${ }^{163}$, A. Cortes-Gonzalez ${ }^{12}$, G. Cortiana ${ }^{101}$, G. Costa ${ }^{91 a}$, M.J. Costa ${ }^{167}$, D. Costanzo ${ }^{139}$, D. Côté ${ }^{8}$, G. $\operatorname{Cottin}^{28}$, G. Cowan ${ }^{77}$, B.E. Cox ${ }^{84}$, K. Cranmer ${ }^{110}$, G. Cree $^{29}$, S. Crépé-Renaudin ${ }^{55}$, F. Crescioli ${ }^{80}$, W.A. Cribbs $^{146 a, 146 b}$, M. Crispin Ortuzar ${ }^{120}$, M. Cristinziani ${ }^{21}$, V. Croft $^{106}$, 
G. Crosetti ${ }^{37 a, 37 b}$, T. Cuhadar Donszelmann ${ }^{139}$, J. Cummings ${ }^{176}$, M. Curatolo ${ }^{47}$, C. Cuthbert $^{150}$, H. Czirr ${ }^{141}$, P. Czodrowski ${ }^{3}$, S. D'Auria ${ }^{53}$, M. D'Onofrio ${ }^{74}$,

M.J. Da Cunha Sargedas De Sousa ${ }^{126 a, 126 b}$, C. Da Via ${ }^{84}$, W. Dabrowski ${ }^{38 a}$, A. Dafinca ${ }^{120}$, T. Dai ${ }^{89}$, O. Dale ${ }^{14}$, F. Dallaire ${ }^{95}$, C. Dallapiccola ${ }^{86}$, M. Dam $^{36}$, J.R. Dandoy ${ }^{31}$, N.P. Dang ${ }^{48}$, A.C. Daniells ${ }^{18}$, M. Danninger ${ }^{168}$, M. Dano Hoffmann ${ }^{136}$, V. Dao ${ }^{48}$, G. Darbo ${ }^{50 a}$, S. Darmora ${ }^{8}$, J. Dassoulas ${ }^{3}$, A. Dattagupta ${ }^{61}$, W. Davey ${ }^{21}$, C. David ${ }^{169}$, T. Davidek ${ }^{129}$, E. Davies ${ }^{120, l}$, M. Davies ${ }^{153}$, P. Davison ${ }^{78}$, Y. Davygora ${ }^{58 a}$, E. Dawe ${ }^{88}$, I. Dawson ${ }^{139}$,

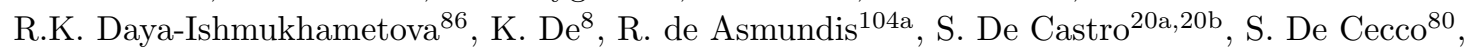
N. De Groot ${ }^{106}$, P. de Jong ${ }^{107}$, H. De la Torre ${ }^{82}$, F. De Lorenzi ${ }^{64}$, L. De Nooij ${ }^{107}$, D. De Pedis ${ }^{132 a}$, A. De Salvo ${ }^{132 a}$, U. De Sanctis ${ }^{149}$, A. De Santo ${ }^{149}$, J.B. De Vivie De Regie ${ }^{117}$, W.J. Dearnaley ${ }^{72}$, R. Debbe ${ }^{25}$, C. Debenedetti ${ }^{137}$, D.V. Dedovich ${ }^{65}$, I. Deigaard ${ }^{107}$, J. Del Peso ${ }^{82}$,

T. Del Prete ${ }^{124 a, 124 b}$, D. Delgove ${ }^{117}$, F. Deliot ${ }^{136}$, C.M. Delitzsch ${ }^{49}$, M. Deliyergiyev ${ }^{75}$, A. Dell'Acqua ${ }^{30}$, L. Dell'Asta ${ }^{22}$, M. Dell'Orso ${ }^{124 a, 124 b}$, M. Della Pietra ${ }^{104 a, j}$, D. della Volpe ${ }^{49}$, M. Delmastro ${ }^{5}$, P.A. Delsart ${ }^{55}$, C. Deluca ${ }^{107}$, D.A. DeMarco ${ }^{158}$, S. Demers ${ }^{176}$, M. Demichev ${ }^{65}$, A. Demilly ${ }^{80}$, S.P. Denisov ${ }^{130}$, D. Derendarz ${ }^{39}$, J.E. Derkaoui ${ }^{135 d}$, F. Derue ${ }^{80}$, P. Dervan ${ }^{74}$, K. Desch $^{21}$, C. Deterre ${ }^{42}$, P.O. Deviveiros ${ }^{30}$, A. Dewhurst ${ }^{131}$, S. Dhaliwal ${ }^{23}$,

A. Di Ciaccio ${ }^{133 a, 133 b}$, L. Di Ciaccio ${ }^{5}$, A. Di Domenico ${ }^{132 a, 132 b}$, C. Di Donato ${ }^{104 a, 104 b}$, A. Di Girolamo ${ }^{30}$, B. Di Girolamo ${ }^{30}$, A. Di Mattia ${ }^{152}$, B. Di Micco ${ }^{134 a, 134 b}$, R. Di Nardo ${ }^{47}$, A. Di Simone ${ }^{48}$, R. Di Sipio ${ }^{158}$, D. Di Valentino ${ }^{29}$, C. Diaconu ${ }^{85}$, M. Diamond ${ }^{158}$, F.A. Dias $^{46}$, M.A. Diaz ${ }^{32 a}$, E.B. Diehl ${ }^{89}$, J. Dietrich ${ }^{16}$, S. Diglio ${ }^{85}$, A. Dimitrievska ${ }^{13}$, J. Dingfelder ${ }^{21}$,

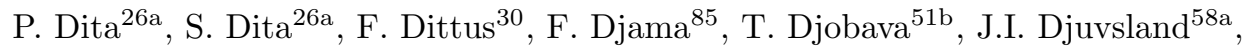
M.A.B. do Vale ${ }^{24 c}$, D. Dobos ${ }^{30}$, M. Dobre ${ }^{26 a}$, C. Doglioni ${ }^{49}$, T. Dohmae ${ }^{155}$, J. Dolejsi ${ }^{129}$, Z. Dolezal ${ }^{129}$, B.A. Dolgoshein ${ }^{98, *}$, M. Donadelli24d ${ }^{24}$ S. Donati ${ }^{124 a, 124 b}$, P. Dondero ${ }^{121 a, 121 b}$,

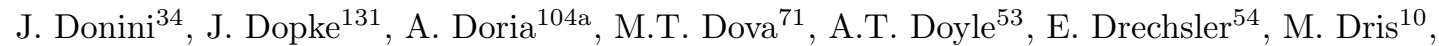
E. Dubreuil ${ }^{34}$, E. Duchovni ${ }^{172}$, G. Duckeck ${ }^{100}$, O.A. Ducu ${ }^{26 a, 85}$, D. Duda ${ }^{175}$, A. Dudarev ${ }^{30}$, L. Duflot ${ }^{117}$, L. Duguid ${ }^{77}$, M. Dührssen ${ }^{30}$, M. Dunford ${ }^{58 a}$, H. Duran Yildiz ${ }^{4 a}$, M. Düren ${ }^{52}$, A. Durglishvili ${ }^{51 b}$, D. Duschinger ${ }^{44}$, M. Dyndal ${ }^{38 a}$, C. Eckardt ${ }^{42}$, K.M. Ecker ${ }^{101}$, R.C. Edgar ${ }^{89}$, W. Edson ${ }^{2}$, N.C. Edwards ${ }^{46}$, W. Ehrenfeld ${ }^{21}$, T. Eifert ${ }^{30}$, G. Eigen ${ }^{14}$, K. Einsweiler ${ }^{15}$, T. Ekelof ${ }^{166}$, M. El Kacimi ${ }^{135 c}$, M. Ellert ${ }^{166}$, S. Elles $^{5}$, F. Ellinghaus ${ }^{83}$, A.A. Elliot ${ }^{169}$, N. Ellis ${ }^{30}$, J. Elmsheuser ${ }^{100}$, M. Elsing ${ }^{30}$, D. Emeliyanov ${ }^{131}$, Y. Enari ${ }^{155}$, O.C. Endner ${ }^{83}$, M. Endo ${ }^{118}$, J. Erdmann ${ }^{43}$, A. Ereditato ${ }^{17}$, G. Ernis ${ }^{175}$, J. Ernst ${ }^{2}$, M. Ernst ${ }^{25}$, S. Errede ${ }^{165}$, E. Ertel ${ }^{83}$, M. Escalier ${ }^{117}$, H. $\operatorname{Esch}^{43}$, C. Escobar ${ }^{125}$, B. Esposito ${ }^{47}$, A.I. Etienvre ${ }^{136}$, E. Etzion ${ }^{153}$, H. Evans ${ }^{61}$, A. Ezhilov ${ }^{123}$, L. Fabbri' ${ }^{20 a}$ 20b ${ }^{2}$ G. Facini ${ }^{31}$, R.M. Fakhrutdinov ${ }^{130}$, S. Falciano ${ }^{132 a}$, R.J. Falla ${ }^{78}$, J. Faltova ${ }^{129}$, Y. Fang ${ }^{33 a}$, M. Fanti ${ }^{91 a, 91 b}$, A. Farbin ${ }^{8}$, A. Farilla ${ }^{134 a}$, T. Farooque ${ }^{12}$, S. Farrell ${ }^{15}$, S.M. Farrington ${ }^{170}$, P. Farthouat ${ }^{30}$, F. Fassi ${ }^{135 e}$, P. Fassnacht ${ }^{30}$, D. Fassouliotis ${ }^{9}$, M. Faucci Giannelli ${ }^{77}$, A. Favareto ${ }^{50 a}$,50b, L. Fayard ${ }^{117}$, P. Federic ${ }^{14 a}$, O.L. Fedin ${ }^{123, m}$, W. Fedorko ${ }^{168}$, S. Feig ${ }^{30}$, L. Feligioni ${ }^{85}$, C. Feng ${ }^{33 d}$, E.J. Feng ${ }^{6}$, H. Feng ${ }^{89}$, A.B. Fenyuk ${ }^{130}$, P. Fernandez Martinez ${ }^{167}$, S. Fernandez Perez ${ }^{30}$, J. Ferrando ${ }^{53}$, A. Ferrari ${ }^{166}$, P. Ferrari ${ }^{107}$, R. Ferrari ${ }^{121 a}$, D.E. Ferreira de Lima ${ }^{53}$, A. Ferrer ${ }^{167}$, D. Ferrere ${ }^{49}$, C. Ferretti ${ }^{89}$,

A. Ferretto Parodi ${ }^{50 a, 50 b}$, M. Fiascaris ${ }^{31}$, F. Fiedler ${ }^{83}$, A. Filipčic ${ }^{75}$, M. Filipuzzi ${ }^{42}$, F. Filthaut ${ }^{106}$, M. Fincke-Keeler ${ }^{169}$, K.D. Finelli1 ${ }^{150}$, M.C.N. Fiolhais ${ }^{126 a, 126 c}$, L. Fiorini ${ }^{167}$, A. Firan ${ }^{40}$,

A. Fischer ${ }^{2}$, C. Fischer ${ }^{12}$, J. Fischer ${ }^{175}$, W.C. Fisher ${ }^{90}$, E.A. Fitzgerald ${ }^{23}$, M. Flechl ${ }^{48}$, I. Fleck ${ }^{141}$, P. Fleischmann ${ }^{89}$, S. Fleischmann ${ }^{175}$, G.T. Fletcher ${ }^{139}$, G. Fletcher ${ }^{76}$, T. Flick ${ }^{175}$, A. Floderus ${ }^{81}$, L.R. Flores Castillo ${ }^{60 a}$, M.J. Flowerdew ${ }^{101}$, A. Formica ${ }^{136}$, A. Forti ${ }^{84}$, D. Fournier ${ }^{117}$, H. Fox ${ }^{72}$, S. Fracchia ${ }^{12}$, P. Francavilla ${ }^{80}$, M. Franchini ${ }^{20 a, 20 b}$, D. Francis ${ }^{30}$, L. Franconi ${ }^{119}$, M. Franklin ${ }^{57}$, M. Fraternali ${ }^{121 a, 121 b}$, D. Freeborn ${ }^{78}$, S.T. French ${ }^{28}$, F. Friedrich ${ }^{44}$, D. Froidevaux ${ }^{30}$, J.A. Frost ${ }^{120}$, C. Fukunaga ${ }^{156}$, E. Fullana Torregrosa ${ }^{83}$, B.G. Fulsom ${ }^{143}$, J. Fuster ${ }^{167}$, C. Gabaldon ${ }^{55}$, O. Gabizon ${ }^{175}$, A. Gabrielli ${ }^{20 a, 20 b}$, A. Gabrielli ${ }^{132 a, 132 b}$, S. Gadatsch ${ }^{107}$, 
S. Gadomski ${ }^{49}$, G. Gagliardi ${ }^{50 a, 50 b}$, P. Gagnon ${ }^{61}$, C. Galea ${ }^{106}$, B. Galhardo ${ }^{126 a, 126 c}$, E.J. Gallas ${ }^{120}$, B.J. Gallop ${ }^{131}$, P. Gallus ${ }^{128}$, G. Galster ${ }^{36}$, K.K. Gan ${ }^{111}$, J. Gao ${ }^{33 b, 85}$, Y. Gao ${ }^{46}$, Y.S. Gao ${ }^{143, e}$, F.M. Garay Walls ${ }^{46}$, F. Garberson ${ }^{176}$, C. García ${ }^{167}$, J.E. García Navarro ${ }^{167}$, M. Garcia-Sciveres ${ }^{15}$, R.W. Gardner ${ }^{31}$, N. Garelli ${ }^{143}$, V. Garonne ${ }^{119}$, C. Gatti ${ }^{47}$, A. Gaudiello50a,50b , G. Gaudio ${ }^{121 a}$, B. Gaur ${ }^{141}$, L. Gauthier ${ }^{95}$, P. Gauzzi ${ }^{132 a, 132 b}$, I.L. Gavrilenko ${ }^{96}$, C. Gay ${ }^{168}$, G. Gaycken ${ }^{21}$, E.N. Gazis ${ }^{10}$, P. Ge ${ }^{33}$, Z. Gecse ${ }^{168}$, C.N.P. Gee ${ }^{131}$, D.A.A. Geerts ${ }^{107}$, Ch. Geich-Gimbel ${ }^{21}$,

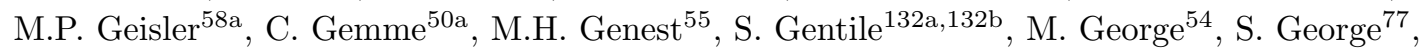
D. Gerbaudo ${ }^{163}$, A. Gershon ${ }^{153}$, H. Ghazlane ${ }^{135 b}$, B. Giacobbe ${ }^{20 a}$, S. Giagu ${ }^{132 a, 132 b}$, V. Giangiobbe ${ }^{12}$, P. Giannetti ${ }^{124 a}, 124 b$, B. Gibbard ${ }^{25}$, S.M. Gibson ${ }^{77}$, M. Gilchriese ${ }^{15}$, T.P.S. Gillam ${ }^{28}$, D. Gillberg ${ }^{30}$, G. Gilles ${ }^{34}$, D.M. Gingrich ${ }^{3, d}$, N. Giokaris ${ }^{9}$, M.P. Giordani ${ }^{164 a, 164 c}$, F.M. Giorgi ${ }^{20 a}$, F.M. Giorgi ${ }^{16}$, P.F. Giraud ${ }^{136}$, P. Giromini ${ }^{47}$, D. Giugni ${ }^{91 a}$, C. Giuliani ${ }^{48}$, M. Giulini ${ }^{58 b}$, B.K. Gjelsten ${ }^{119}$, S. Gkaitatzis ${ }^{154}$, I. Gkialas ${ }^{154}$, E.L. Gkougkousis ${ }^{117}$, L.K. Gladilin ${ }^{99}$, C. Glasman ${ }^{82}$, J. Glatzer ${ }^{30}$, P.C.F. Glaysher ${ }^{46}$, A. Glazov ${ }^{42}$, M. Goblirsch-Kolb ${ }^{101}$, J.R. Goddard ${ }^{76}$, J. Godlewski ${ }^{39}$, S. Goldfarb ${ }^{89}$, T. Golling ${ }^{49}$, D. Golubkov ${ }^{130}$, A. Gomes ${ }^{126 a, 126 b, 126 d}$, R. Gonçalo ${ }^{126 a}$, J. Goncalves Pinto Firmino Da Costa ${ }^{136}$, L. Gonella ${ }^{21}$, S. González de la Hoz ${ }^{167}$, G. Gonzalez Parra ${ }^{12}$, S. Gonzalez-Sevilla ${ }^{49}$, L. Goossens ${ }^{30}$, P.A. Gorbounov ${ }^{7}$, H.A. Gordon ${ }^{25}$, I. Gorelov ${ }^{105}$, B. Gorini ${ }^{30}$, E. Gorini ${ }^{73 a, 73 b}$, A. Gorišek ${ }^{75}$, E. Gornicki ${ }^{39}$, A.T. Goshaw ${ }^{45}$, C. Gössling ${ }^{43}$, M.I. Gostkin ${ }^{65}$, D. Goujdami ${ }^{135 c}$, A.G. Goussiou ${ }^{138}$, N. Govender ${ }^{145 b}$, H.M.X. Grabas ${ }^{137}$, L. Graber ${ }^{54}$, I. Grabowska-Bold ${ }^{38 a}$, P. Grafström ${ }^{20 a, 20 b}$ K-J. Grahn ${ }^{42}$, J. Gramling ${ }^{49}$, E. Gramstad ${ }^{119}$, S. Grancagnolo ${ }^{16}$, V. Grassi ${ }^{148}$, V. Gratchev ${ }^{123}$, H.M. Gray ${ }^{30}$, E. Graziani ${ }^{134 a}$, Z.D. Greenwood ${ }^{79, n}$, K. Gregersen ${ }^{78}$, I.M. Gregor ${ }^{42}$, P. Grenier ${ }^{143}$, J. Griffiths ${ }^{8}$, A.A. Grillo ${ }^{137}$, K. Grimm ${ }^{72}$, S. Grinstein ${ }^{12, o}$, Ph. Gris ${ }^{34}$, J.-F. Grivaz ${ }^{117}$, J.P. Grohs ${ }^{44}$, A. Grohsjean ${ }^{42}$, E. Gross ${ }^{172}$, J. Grosse-Knetter ${ }^{54}$, G.C. Grossi ${ }^{79}$, Z.J. Grout ${ }^{149}$, L. Guan ${ }^{33 b}$, J. Guenther ${ }^{128}$, F. Guescini ${ }^{49}$, D. Guest ${ }^{176}$, O. Gueta ${ }^{153}$, E. Guido ${ }^{50 a, 50 b}$, T. Guillemin ${ }^{117}$, S. Guindon ${ }^{2}$, U. Gul ${ }^{53}$, C. Gumpert ${ }^{44}$, J. Guo ${ }^{33 e}$, S. Gupta $^{120}$, P. Gutierrez ${ }^{113}$, N.G. Gutierrez Ortiz ${ }^{53}$, C. Gutschow ${ }^{44}$, C. Guyot ${ }^{136}$, C. Gwenlan ${ }^{120}$, C.B. Gwilliam ${ }^{74}$, A. Haas ${ }^{110}$, C. Haber ${ }^{15}$, H.K. Hadavand ${ }^{8}$, N. Haddad ${ }^{135 e}$, P. Haefner ${ }^{21}$, S. Hageböck ${ }^{21}$, Z. Hajduk ${ }^{39}$, H. Hakobyan ${ }^{177}$, M. Haleem ${ }^{42}$, J. Haley ${ }^{114}$, D. Hall ${ }^{120}$, G. Halladjian ${ }^{90}$, G.D. Hallewell ${ }^{85}$, K. Hamacher ${ }^{175}$, P. Hamal ${ }^{115}$, K. Hamano ${ }^{169}$, M. Hamer ${ }^{54}$, A. Hamilton ${ }^{145 a}$, S. Hamilton ${ }^{161}$, G.N. Hamity ${ }^{145 c}$, P.G. Hamnett ${ }^{42}$, L. Han ${ }^{33 b}$, K. Hanagaki ${ }^{118}$, K. Hanawa ${ }^{155}$, M. Hance ${ }^{15}$, P. Hanke ${ }^{58 a}$, R. Hanna ${ }^{136}$, J.B. Hansen ${ }^{36}$, J.D. Hansen ${ }^{36}$, M.C. Hansen ${ }^{21}$,

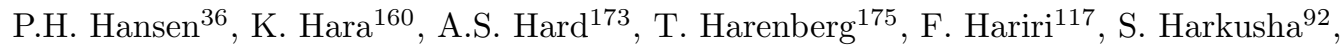
R.D. Harrington ${ }^{46}$, P.F. Harrison ${ }^{170}$, F. Hartjes ${ }^{107}$, M. Hasegawa ${ }^{67}$, S. Hasegawa ${ }^{103}$, Y. Hasegawa ${ }^{140}$, A. Hasib ${ }^{113}$, S. Hassani ${ }^{136}$, S. Haug ${ }^{17}$, R. Hauser ${ }^{90}$, L. Hauswald ${ }^{44}$, M. Havranek ${ }^{127}$, C.M. Hawkes ${ }^{18}$, R.J. Hawkings ${ }^{30}$, A.D. Hawkins ${ }^{81}$, T. Hayashi ${ }^{160}$, D. Hayden ${ }^{90}$,

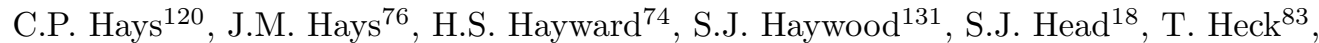
V. Hedberg ${ }^{81}$, L. Heelan ${ }^{8}$, S. Heim ${ }^{122}$, T. Heim ${ }^{175}$, B. Heinemann ${ }^{15}$, L. Heinrich ${ }^{110}$, J. Hejbal ${ }^{127}$, L. Helary ${ }^{22}$, S. Hellman ${ }^{146 a, 146 b}$, D. Hellmich ${ }^{21}$, C. Helsens ${ }^{30}$, J. Henderson ${ }^{120}$,

R.C.W. Henderson ${ }^{72}$, Y. Heng ${ }^{173}$, C. Hengler ${ }^{42}$, A. Henrichs ${ }^{176}$, A.M. Henriques Correia ${ }^{30}$, S. Henrot-Versille ${ }^{117}$, G.H. Herbert ${ }^{16}$, Y. Hernández Jiménez ${ }^{167}$, R. Herrberg-Schubert ${ }^{16}$,

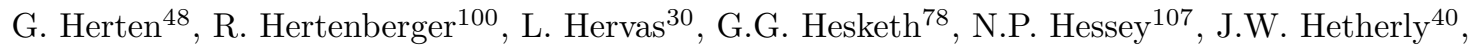
R. Hickling ${ }^{76}$, E. Higón-Rodriguez ${ }^{167}$, E. Hill ${ }^{169}$, J.C. Hill ${ }^{28}$, K.H. Hiller ${ }^{42}$, S.J. Hillier ${ }^{18}$, I. Hinchliffe ${ }^{15}$, E. Hines ${ }^{122}$, R.R. Hinman ${ }^{15}$, M. Hirose ${ }^{157}$, D. Hirschbuehl ${ }^{175}$, J. Hobbs ${ }^{148}$, N. Hod ${ }^{107}$, M.C. Hodgkinson ${ }^{139}$, P. Hodgson ${ }^{139}$, A. Hoecker ${ }^{30}$, M.R. Hoeferkamp ${ }^{105}$, F. Hoenig ${ }^{100}$, M. Hohlfeld ${ }^{83}$, D. Hohn ${ }^{21}$, T.R. Holmes ${ }^{15}$, M. Homann ${ }^{43}$, T.M. Hong ${ }^{125}$,

L. Hooft van Huysduynen ${ }^{110}$, W.H. Hopkins ${ }^{116}$, Y. Horii ${ }^{103}$, A.J. Horton ${ }^{142}$, J-Y. Hostachy ${ }^{55}$, S. Hou ${ }^{151}$, A. Hoummada ${ }^{135 a}$, J. Howard ${ }^{120}$, J. Howarth ${ }^{42}$, M. Hrabovsky ${ }^{115}$, I. Hristova ${ }^{16}$, J. Hrivnac ${ }^{117}$, T. Hryn'ova ${ }^{5}$, A. Hrynevich ${ }^{93}$, C. Hsu ${ }^{145 c}$, P.J. Hsu ${ }^{151, p}$, S.-C. Hsu ${ }^{138}$, D. Hu ${ }^{35}$, 


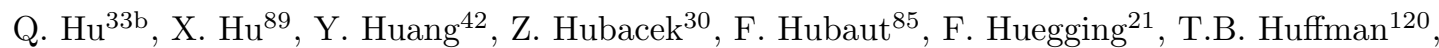
E.W. Hughes ${ }^{35}$, G. Hughes ${ }^{72}$, M. Huhtinen ${ }^{30}$, T.A. Hülsing ${ }^{83}$, N. Huseynov ${ }^{65, b}$, J. Huston ${ }^{90}$, J. Huth ${ }^{57}$, G. Iacobucci ${ }^{49}$, G. Iakovidis ${ }^{25}$, I. Ibragimov ${ }^{141}$, L. Iconomidou-Fayard ${ }^{117}$, E. Ideal ${ }^{176}$, Z. Idrissi ${ }^{135 e}$, P. Iengo ${ }^{30}$, O. Igonkina ${ }^{107}$, T. Iizawa ${ }^{171}$, Y. Ikegami ${ }^{66}$, K. Ikematsu $^{141}$, M. Ikeno ${ }^{66}$, Y. Ilchenko ${ }^{31, q}$, D. Iliadis ${ }^{154}$, N. Ilic ${ }^{158}$, Y. Inamaru ${ }^{67}$, T. Ince ${ }^{101}$, P. Ioannou ${ }^{9}$, M. Iodice $^{134 a}$, K. Iordanidou ${ }^{35}, \mathrm{~V}$. Ippolito ${ }^{57}$, A. Irles Quiles ${ }^{167}$, C. Isaksson ${ }^{166}$, M. Ishino ${ }^{68}$, M. Ishitsuka ${ }^{157}$, R. Ishmukhametov ${ }^{111}$, C. Issever ${ }^{120}$, S. Istin ${ }^{19 a}$, J.M. Iturbe Ponce ${ }^{84}$, R. Iuppa ${ }^{133 a, 133 b}$, J. Ivarsson ${ }^{81}$, W. Iwanski ${ }^{39}$, H. Iwasaki ${ }^{66}$, J.M. Izen ${ }^{41}$, V. Izzo ${ }^{104 a}$, S. Jabbar ${ }^{3}$, B. Jackson ${ }^{122}$, M. Jackson ${ }^{74}$, P. Jackson ${ }^{1}$, M.R. Jaekel ${ }^{30}$, V. Jain ${ }^{2}$, K. Jakobs ${ }^{48}$, S. Jakobsen ${ }^{30}$, T. Jakoubek ${ }^{127}$, J. Jakubek ${ }^{128}$, D.O. Jamin ${ }^{151}$, D.K. Jana ${ }^{79}$, E. Jansen ${ }^{78}$, R.W. Jansky ${ }^{62}$, J. Janssen ${ }^{21}$, M. Janus ${ }^{170}$, G. Jarlskog ${ }^{81}$, N. Javadov ${ }^{65, b}$, T. Javưrek ${ }^{48}$, L. Jeanty ${ }^{15}$, J. Jejelava ${ }^{51 a, r}$, G.-Y. Jeng ${ }^{150}$, D. Jennens ${ }^{88}$, P. Jenni ${ }^{48, s}$, J. Jentzsch ${ }^{43}$, C. Jeske ${ }^{170}$, S. Jézéquel ${ }^{5}$, H. Ji ${ }^{173}$, J. Jia ${ }^{148}$, Y. Jiang ${ }^{33 b}$, S. Jiggins ${ }^{78}$, J. Jimenez Pena ${ }^{167}$, S. Jin ${ }^{33 a}$, A. Jinaru ${ }^{26 a}$, O. Jinnouchi ${ }^{157}$, M.D. Joergensen ${ }^{36}$, P. Johansson ${ }^{139}$, K.A. Johns ${ }^{7}$, K. Jon-And ${ }^{146 a, 146 b}$, G. Jones ${ }^{170}$,

R.W.L. Jones ${ }^{72}$, T.J. Jones ${ }^{74}$, J. Jongmanns ${ }^{58 a}$, P.M. Jorge ${ }^{126 a, 126 b}$, K.D. Joshi ${ }^{84}$, J. Jovicevic ${ }^{159 a}$, X. Ju ${ }^{173}$, C.A. Jung ${ }^{43}$, P. Jussel ${ }^{62}$, A. Juste Rozas ${ }^{12, o}$, M. Kaci ${ }^{167}$, A. Kaczmarska ${ }^{39}$, M. Kado ${ }^{117}$, H. Kagan ${ }^{111}$, M. Kagan ${ }^{143}$, S.J. Kahn ${ }^{85}$, E. Kajomovitz ${ }^{45}$, C.W. Kalderon ${ }^{120}$, S. Kama ${ }^{40}$,

A. Kamenshchikov ${ }^{130}$, N. Kanaya ${ }^{155}$, M. Kaneda ${ }^{30}$, S. Kaneti ${ }^{28}$, V.A. Kantserov ${ }^{98}$, J. Kanzaki ${ }^{66}$, B. Kaplan ${ }^{110}$, A. Kapliy ${ }^{31}$, D. Kar ${ }^{53}$, K. Karakostas ${ }^{10}$, A. Karamaoun ${ }^{3}$, N. Karastathis ${ }^{10,107}$, M.J. Kareem ${ }^{54}$, M. Karnevskiy ${ }^{83}$, S.N. Karpov ${ }^{65}$, Z.M. Karpova ${ }^{65}$, K. Karthik ${ }^{110}$,

V. Kartvelishvili ${ }^{72}$, A.N. Karyukhin ${ }^{130}$, L. Kashif ${ }^{173}$, R.D. Kass ${ }^{111}$, A. $\operatorname{Kastanas}^{14}$, Y. Kataoka ${ }^{155}$, A. Katre ${ }^{49}$, J. Katzy ${ }^{42}$, K. Kawagoe ${ }^{70}$, T. Kawamoto ${ }^{155}$, G. Kawamura ${ }^{54}$, S. Kazama ${ }^{155}$, V.F. Kazanin ${ }^{109, c}$, M.Y. Kazarinov ${ }^{65}$, R. Keeler ${ }^{169}$, R. Kehoe ${ }^{40}$, J.S. Keller ${ }^{42}$, J.J. Kempster ${ }^{77}$, H. Keoshkerian ${ }^{84}$, O. Kepka ${ }^{127}$, B.P. Kerševan ${ }^{75}$, S. Kersten ${ }^{175}$, R.A. Keyes ${ }^{87}$, F. Khalil-zada ${ }^{11}$, H. Khandanyan ${ }^{146 a, 146 b}$, A. Khanov ${ }^{114}$, A.G. Kharlamov ${ }^{109, c}$, T.J. Khoo ${ }^{28}$, V. Khovanskiy ${ }^{97}$, E. Khramov ${ }^{65}$, J. Khubua ${ }^{51 b, t}$, H.Y. Kim ${ }^{8}$, H. Kim${ }^{146 a, 146 b}$, S.H. Kim ${ }^{160}$, Y. Kim ${ }^{31}$, N. Kimura ${ }^{154}$, O.M. Kind ${ }^{16}$, B.T. King ${ }^{74}$, M. King ${ }^{167}$, R.S.B. King ${ }^{120}$, S.B. King ${ }^{168}$, J. Kirk ${ }^{131}$, A.E. Kiryunin ${ }^{101}$, T. Kishimoto ${ }^{67}$, D. Kisielewska ${ }^{38 a}$, F. Kiss ${ }^{48}$, K. Kiuchi ${ }^{160}$, O. Kivernyk ${ }^{136}$, E. Kladiva ${ }^{144 b}$, M.H. Klein ${ }^{35}$, M. Klein ${ }^{74}$, U. Klein ${ }^{74}$, K. Kleinknecht ${ }^{83}$, P. Klimek ${ }^{146 a, 146 b}$, A. Klimentov ${ }^{25}$, R. Klingenberg ${ }^{43}$, J.A. Klinger ${ }^{84}$, T. Klioutchnikova ${ }^{30}$, E.-E. Kluge ${ }^{58 a}$, P. Kluit ${ }^{107}$, S. Kluth ${ }^{101}$, E. Kneringer ${ }^{62}$, E.B.F.G. Knoops ${ }^{85}$, A. Knue ${ }^{53}$, A. Kobayashi ${ }^{155}$, D. Kobayashi ${ }^{157}$, T. Kobayashi ${ }^{155}$, M. Kobel ${ }^{44}$, M. Kocian ${ }^{143}$, P. Kodys ${ }^{129}$, T. Koffas ${ }^{29}$, E. Koffeman ${ }^{107}$, L.A. Kogan ${ }^{120}$, S. Kohlmann ${ }^{175}$, Z. Kohout ${ }^{128}$, T. Kohriki ${ }^{66}$, T. Koi ${ }^{143}$, H. Kolanoski ${ }^{16}$, I. Koletsou ${ }^{5}$, A.A. Komar ${ }^{96, *}$, Y. Komori ${ }^{155}$, T. Kondo ${ }^{66}$, N. Kondrashova ${ }^{42}$, K. Köneke ${ }^{48}$, A.C. König ${ }^{106}$, S. König ${ }^{83}$, T. Kono ${ }^{66, u}$, R. Konoplich ${ }^{110, v}$, N. Konstantinidis ${ }^{78}$, R. Kopeliansky ${ }^{152}$, S. Koperny ${ }^{38 a}$, L. Köpke ${ }^{83}$, A.K. Kopp ${ }^{48}$, K. Korcyl ${ }^{39}$, K. Kordas ${ }^{154}$, A. Korn ${ }^{78}$, A.A. Korol ${ }^{109, c}$, I. Korolkov ${ }^{12}$, E.V. Korolkova ${ }^{139}$, O. Kortner ${ }^{101}$, S. Kortner ${ }^{101}$, T. Kosek ${ }^{129}$, V.V. Kostyukhinn ${ }^{21}$, V.M. Kotov ${ }^{65}$, A. Kotwal ${ }^{45}$, A. Kourkoumeli-Charalampidi ${ }^{154}$, C. Kourkoumelis ${ }^{9}$, V. Kouskoura ${ }^{25}$, A. Koutsman ${ }^{159 a}$, R. Kowalewski ${ }^{169}$, T.Z. Kowalski ${ }^{38 a}$, W. Kozanecki ${ }^{136}$, A.S. Kozhin ${ }^{130}$, V.A. Kramarenko ${ }^{99}$, G. Kramberger ${ }^{75}$, D. Krasnopevtsev ${ }^{98}$, M.W. Krasny ${ }^{80}$, A. Krasznahorkay ${ }^{30}$, J.K. Kraus ${ }^{21}$, A. Kravchenko ${ }^{25}$, S. Kreiss ${ }^{110}$, M. Kretz ${ }^{58 c}$, J. Kretzschmar ${ }^{74}$, K. Kreutzfeldt ${ }^{52}$, P. Krieger ${ }^{158}$, K. Krizka ${ }^{31}$, K. Kroeninger ${ }^{43}$, H. Kroha ${ }^{101}$, J. Kroll ${ }^{122}$, J. Kroseberg ${ }^{21}$, J. Krstic ${ }^{13}$, U. Kruchonak ${ }^{65}$, H. Krüger ${ }^{21}$, N. Krumnack ${ }^{64}$, Z.V. Krumshteyn ${ }^{65}$, A. Kruse ${ }^{173}$, M.C. Kruse ${ }^{45}$, M. Kruskal ${ }^{22}$, T. Kubota ${ }^{88}$, H. Kucuk ${ }^{78}$, S. Kuday ${ }^{4 c}$, S. Kuehn ${ }^{48}$, A. Kugel ${ }^{58 c}$, F. Kuger ${ }^{174}$, A. Kuhl ${ }^{137}$, T. Kuhl ${ }^{42}$, V. Kukhtin ${ }^{65}$, Y. Kulchitsky ${ }^{92}$, S. Kuleshov ${ }^{32 b}$, M. Kuna ${ }^{132 a, 132 b}$, T. Kunigo ${ }^{68}$, A. Kupco ${ }^{127}$, H. Kurashige ${ }^{67}$, Y.A. Kurochkin ${ }^{92}$, R. Kurumida ${ }^{67}$, V. Kus ${ }^{127}$, E.S. Kuwertz ${ }^{169}$, M. Kuze ${ }^{157}$, J. Kvita ${ }^{115}$, T. Kwan ${ }^{169}$, D. Kyriazopoulos ${ }^{139}$, A. La Rosa ${ }^{49}$, J.L. La Rosa Navarro ${ }^{24 d}$, L. La Rotonda ${ }^{37 a, 37 b}$, 
C. Lacasta ${ }^{167}$, F. Lacava ${ }^{132 a, 132 b}$, J. Lace ${ }^{29}$, H. Lacker $^{16}$, D. Lacour ${ }^{80}$, V.R. Lacuesta ${ }^{167}$, E. Ladygin ${ }^{65}$, R. Lafaye ${ }^{5}$, B. Laforge ${ }^{80}$, T. Lagouri ${ }^{176}$, S. Lai ${ }^{48}$, L. Lambourne ${ }^{78}$, S. Lammers ${ }^{61}$, C.L. Lampen ${ }^{7}$, W. Lampl ${ }^{7}$, E. Lançon ${ }^{136}$, U. Landgraf ${ }^{48}$, M.P.J. Landon ${ }^{76}$, V.S. Lang ${ }^{58 a}$, J.C. Lange ${ }^{12}$, A.J. Lankford ${ }^{163}$, F. Lanni ${ }^{25}$, K. Lantzsch ${ }^{30}$, S. Laplace ${ }^{80}$, C. Lapoire ${ }^{30}$, J.F. Laporte ${ }^{136}$, T. Lari ${ }^{91 a}$, F. Lasagni Manghi ${ }^{20 a, 20 b}$, M. Lassnig ${ }^{30}$, P. Laurelli ${ }^{47}$, W. Lavrijsen ${ }^{15}$, A.T. Law ${ }^{137}$, P. Laycock ${ }^{74}$, O. Le Dortz ${ }^{80}$, E. Le Guirriec ${ }^{85}$, E. Le Menedeu ${ }^{12}$, M. LeBlanc ${ }^{169}$, T. LeCompte ${ }^{6}$, F. Ledroit-Guillon ${ }^{55}$, C.A. Lee ${ }^{145 b}$, S.C. Lee ${ }^{151}$, L. Lee ${ }^{1}$, G. Lefebvre ${ }^{80}$, M. Lefebvre ${ }^{169}$, F. Legger ${ }^{100}$, C. Leggett ${ }^{15}$, A. Lehan ${ }^{74}$, G. Lehmann Miotto ${ }^{30}$, X. Lei ${ }^{7}$, W.A. Leight ${ }^{29}$, A. Leisos ${ }^{154, w}$, A.G. Leister ${ }^{176}$, M.A.L. Leite ${ }^{24 d}$, R. Leitner ${ }^{129}$, D. Lellouch ${ }^{172}$, B. Lemmer ${ }^{54}$, K.J.C. Leney ${ }^{78}$, T. Lenz ${ }^{21}$, B. Lenzi ${ }^{30}$, R. Leone ${ }^{7}$, S. Leone ${ }^{124 a, 124 b}$, C. Leonidopoulos ${ }^{46}$, S. Leontsinis ${ }^{10}$, C. Leroy ${ }^{95}$, C.G. Lester $^{28}$, M. Levchenko ${ }^{123}$, J. Levêque ${ }^{5}$, D. Levin ${ }^{89}$, L.J. Levinson ${ }^{172}$, M. Levy ${ }^{18}$, A. Lewis ${ }^{120}$, A.M. Leyko ${ }^{21}$, M. Leyton ${ }^{41}$, B. Li ${ }^{33 b, x}$, H. $\mathrm{Li}^{148}$, H.L. $\mathrm{Li}^{31}$, L. $\mathrm{Li}^{45}$, L. Li ${ }^{33 \mathrm{e}}$, S. $\mathrm{Li}^{45}$, Y. $\mathrm{Li}^{33 \mathrm{c}, y}$, Z. Liang ${ }^{137}, \mathrm{H} . \mathrm{LiaO}^{34}$, B. Liberti ${ }^{133 \mathrm{a}}$,

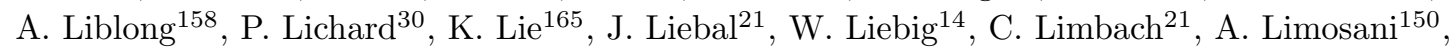
S.C. $\operatorname{Lin}^{151, z}$, T.H. $\operatorname{Lin}^{83}$, F. Linde ${ }^{107}$, B.E. Lindquist ${ }^{148}$, J.T. Linnemann ${ }^{90}$, E. Lipeles ${ }^{122}$, A. Lipniacka ${ }^{14}$, M. Lisovyi ${ }^{42}$, T.M. Liss ${ }^{165}$, D. Lissauer ${ }^{25}$, A. Lister ${ }^{168}$, A.M. Litke ${ }^{137}$,

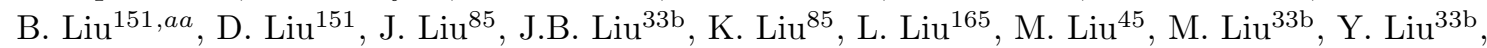
M. Livan ${ }^{121 \mathrm{a}, 121 \mathrm{~b}}$, A. Lleres ${ }^{55}$, J. Llorente Merino ${ }^{82}$, S.L. Lloyd $^{76}$, F. Lo Sterzo ${ }^{151}$, E. Lobodzinska ${ }^{42}$, P. Loch ${ }^{7}$, W.S. Lockman ${ }^{137}$, F.K. Loebinger ${ }^{84}$, A.E. Loevschall-Jensen ${ }^{36}$, A. Loginov ${ }^{176}$, T. Lohse ${ }^{16}$, K. Lohwasser ${ }^{42}$, M. Lokajicek ${ }^{127}$, B.A. Long ${ }^{22}$, J.D. Long ${ }^{89}$, R.E. Long ${ }^{72}$, K.A. Looper ${ }^{111}$, L. Lopes ${ }^{126 a}$, D. Lopez Mateos ${ }^{57}$, B. Lopez Paredes ${ }^{139}$, I. Lopez Paz ${ }^{12}$, J. Lorenz ${ }^{100}$, N. Lorenzo Martinez ${ }^{61}$, M. Losada $^{162}$, P. Loscutoff ${ }^{15}$, P.J. Lösel ${ }^{100}$, X. Lou $^{33 a}$, A. Lounis ${ }^{117}$, J. Love ${ }^{6}$, P.A. Love ${ }^{72}$, N. Lu ${ }^{89}$, H.J. Lubatti ${ }^{138}$, C. Luci ${ }^{132 a, 132 b}$,

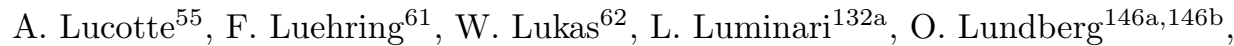
B. Lund-Jensen ${ }^{147}$, D. Lynn ${ }^{25}$, R. Lysak ${ }^{127}$, E. Lytken ${ }^{81}$, H. Ma ${ }^{25}$, L.L. Ma ${ }^{33 d}$, G. Maccarrone ${ }^{47}$, A. Macchiolo ${ }^{101}$, C.M. Macdonald ${ }^{139}$, J. Machado Miguens ${ }^{122,126 b}$, D. Macina ${ }^{30}$, D. Madaffari ${ }^{85}$, R. $\operatorname{Madar}^{34}$, H.J. Maddocks ${ }^{72}$, W.F. Mader ${ }^{44}$, A. Madsen ${ }^{166}$, S. Maeland ${ }^{14}$, T. Maeno ${ }^{25}$, A. Maevskiy ${ }^{99}$, E. Magradze ${ }^{54}$, K. Mahboubi ${ }^{48}$, J. Mahlstedt ${ }^{107}$, C. Maiani ${ }^{136}$, C. Maidantchik ${ }^{24 a}$, A.A. Maier ${ }^{101}$, T. Maier ${ }^{100}$, A. Maio ${ }^{126 a, 126 b, 126 d}$, S. Majewski ${ }^{116}$, Y. Makida ${ }^{66}$, N. Makovec ${ }^{117}$, B. Malaescu ${ }^{80}$, Pa. Malecki ${ }^{39}$, V.P. Maleev ${ }^{123}$, F. Malek ${ }^{55}$, U. Mallik ${ }^{63}$, D. Malon ${ }^{6}$, C. Malone ${ }^{143}$, S. Maltezos ${ }^{10}$, V.M. Malyshev ${ }^{109}$, S. Malyukov ${ }^{30}$, J. Mamuzic ${ }^{42}$, G. Mancini ${ }^{47}$, B. Mandelli ${ }^{30}$, L. Mandelli ${ }^{91 a}$, I. Mandić ${ }^{75}$, R. Mandrysch ${ }^{63}$, J. Maneira ${ }^{126 a, 126 b}$, A. Manfredini1 ${ }^{101}$, L. Manhaes de Andrade Filho ${ }^{24 b}$, J. Manjarres Ramos ${ }^{159 b}$, A. Mann ${ }^{100}$, P.M. Manning ${ }^{137}$, A. Manousakis-Katsikakis ${ }^{9}$, B. Mansoulie ${ }^{136}$, R. Mantifel ${ }^{87}$, M. Mantoani ${ }^{54}$, L. Mapelli $^{30}$, L. March ${ }^{145 c}$, G. Marchiori ${ }^{80}$, M. Marcisovsky ${ }^{127}$, C.P. Marino ${ }^{169}$, M. Marjanovic ${ }^{13}$, F. Marroquim ${ }^{24 a}$, S.P. Marsden ${ }^{84}$, Z. Marshall ${ }^{15}$, L.F. Marti ${ }^{17}$, S. Marti-Garcia ${ }^{167}$, B. Martin ${ }^{90}$, T.A. Martin ${ }^{170}$, V.J. Martin ${ }^{46}$, B. Martin dit Latour ${ }^{14}$, M. Martinez ${ }^{12, o}$, S. Martin-Haugh ${ }^{131}$, V.S. Martoiu ${ }^{26 a}$, A.C. Martyniuk ${ }^{78}$, M. Marx ${ }^{138}$, F. Marzano ${ }^{132 a}$, A. Marzin ${ }^{30}$, L. Masetti ${ }^{83}$, T. Mashimo ${ }^{155}$, R. Mashinistov ${ }^{96}$, J. Masik ${ }^{84}$, A.L. Maslennikov ${ }^{109, c}$, I. Massa ${ }^{20 a, 20 b}$, L. Massa ${ }^{20 a, 20 b}$, N. Massol ${ }^{5}$, P. Mastrandrea ${ }^{148}$, A. Mastroberardino ${ }^{37 a, 37 b}$, T. Masubuchi ${ }^{155}$, P. Mättig ${ }^{175}$, J. Mattmann ${ }^{83}$, J. Maurer ${ }^{26 a}$, S.J. Maxfield ${ }^{74}$, D.A. Maximov ${ }^{109, c}$, R. Mazini ${ }^{151}$, S.M. Mazza ${ }^{91 a, 91 b}$, L. Mazzaferro ${ }^{133 a, 133 b}$, G. Mc Goldrick ${ }^{158}$, S.P. Mc Kee ${ }^{89}$, A. McCarn ${ }^{89}$, R.L. McCarthy ${ }^{148}$, T.G. McCarthy ${ }^{29}$, N.A. McCubbin ${ }^{131}$, K.W. McFarlane ${ }^{56, *}$, J.A. Mcfayden ${ }^{78}$, G. Mchedlidze ${ }^{54}$, S.J. McMahon ${ }^{131}$, R.A. McPherson ${ }^{169, k}$, M. Medinnis ${ }^{42}$, S. Meehan ${ }^{145 a}$, S. Mehlhase ${ }^{100}$, A. Mehta $^{74}$, K. Meier ${ }^{58 a}$, C. Meineck ${ }^{100}$, B. Meirose ${ }^{41}$, B.R. Mellado Garcia ${ }^{145 c}$, F. Meloni ${ }^{17}$, A. Mengarelli ${ }^{20 a}, 20 \mathrm{~b}$, S. Menke ${ }^{101}$, E. Meoni ${ }^{161}$, K.M. Mercurio ${ }^{57}$, S. Mergelmeyer ${ }^{21}$, P. Mermod ${ }^{49}$, L. Merola ${ }^{104 a, 104 b}$, C. Meroni ${ }^{91 a}$, F.S. Merritt ${ }^{31}$, A. Messina ${ }^{132 a, 132 b}$, J. Metcalfe ${ }^{25}$, A.S. Mete $^{163}$, C. Meyer $^{83}$, C. Meyer ${ }^{122}$, J-P. Meyer ${ }^{136}$, J. Meyer ${ }^{107}$, R.P. Middleton ${ }^{131}$, 
S. Miglioranzi ${ }^{164 a, 164 c}$, L. Mijović ${ }^{21}$, G. Mikenberg ${ }^{172}$, M. Mikestikova ${ }^{127}$, M. Mikuž ${ }^{75}$, M. Milesi ${ }^{88}$, A. Milic ${ }^{30}$, D.W. Miller ${ }^{31}$, C. Mills ${ }^{46}$, A. Milov ${ }^{172}$, D.A. Milstead ${ }^{146 a, 146 b}$, A.A. Minaenko ${ }^{130}$, Y. Minami ${ }^{155}$, I.A. Minashvili ${ }^{65}$, A.I. Mincer ${ }^{110}$, B. Mindur ${ }^{38 a}$, M. Mineev ${ }^{65}$, Y. Ming ${ }^{173}$, L.M. Mir ${ }^{12}$, T. Mitani ${ }^{171}$, J. Mitrevski ${ }^{100}$, V.A. Mitsou ${ }^{167}$, A. Miucci ${ }^{49}$, P.S. Miyagawa ${ }^{139}$, J.U. Mjörnmark ${ }^{81}$, T. Moa ${ }^{146 a, 146 b}$, K. Mochizuki ${ }^{85}$, S. Mohapatra ${ }^{35}$, W. Mohr ${ }^{48}$, S. Molander ${ }^{146 a, 146 b}$, R. Moles-Valls ${ }^{167}$, K. Mönig ${ }^{42}$, C. Monini' ${ }^{55}$, J. Monk ${ }^{36}$, E. Monnier ${ }^{85}$, J. Montejo Berlingen ${ }^{12}$, F. Monticelli ${ }^{71}$, S. Monzani ${ }^{132 a, 132 b}$, R.W. Moore ${ }^{3}$, N. Morange ${ }^{117}$, D. Moreno ${ }^{162}$, M. Moreno Llácer ${ }^{54}$, P. Morettini $^{50 a}$, M. Morgenstern ${ }^{44}$, M. Moriii ${ }^{57}$, M. Morinaga ${ }^{155}$, V. Morisbak ${ }^{119}$, S. Moritz ${ }^{83}$, A.K. Morley ${ }^{147}$, G. Mornacchi ${ }^{30}$, J.D. Morris ${ }^{76}$, S.S. Mortensen ${ }^{36}$, A. Morton ${ }^{53}$, L. Morvaj ${ }^{103}$, M. Mosidze ${ }^{51 b}$, J. Moss ${ }^{111}$, K. Motohashi ${ }^{157}$, R. Mount ${ }^{143}$, E. Mountricha ${ }^{25}$, S.V. Mouraviev ${ }^{96, *}$, E.J.W. Moyse ${ }^{86}$, S. Muanza ${ }^{85}$, R.D. Mudd ${ }^{18}$, F. Mueller ${ }^{101}$, J. Mueller ${ }^{125}$, K. Mueller ${ }^{21}$, R.S.P. Mueller ${ }^{100}$, T. Mueller ${ }^{28}$, D. Muenstermann ${ }^{49}$, P. Mullen ${ }^{53}$, Y. Munwes ${ }^{153}$, J.A. Murillo Quijada ${ }^{18}$, W.J. Murray ${ }^{170,131}$, H. Musheghyan ${ }^{54}$, E. Musto ${ }^{152}$, A.G. Myagkov ${ }^{130, a b}$, M. Myska ${ }^{128}$, O. Nackenhorst ${ }^{54}$, J. Nadal ${ }^{54}$, K. Nagai ${ }^{120}$, R. Nagai ${ }^{157}$, Y. Nagai ${ }^{85}$, K. Nagano ${ }^{66}$, A. Nagarkar ${ }^{111}$, Y. Nagasaka ${ }^{59}$, K. Nagata ${ }^{160}$, M. Nagel ${ }^{101}$, E. Nagy ${ }^{85}$, A.M. Nairz ${ }^{30}$, Y. Nakahama ${ }^{30}$, K. Nakamura ${ }^{66}$, T. Nakamura ${ }^{155}$, I. Nakano ${ }^{112}$, H. Namasivayam ${ }^{41}$, R.F. Naranjo Garcia ${ }^{42}$, R. Narayan ${ }^{31}$, T. Naumann ${ }^{42}$, G. Navarro ${ }^{162}$, R. Nayyar ${ }^{7}$, H.A. Neal ${ }^{89}$, P.Yu. Nechaeva ${ }^{96}$, T.J. Neep ${ }^{84}$, P.D. $\mathrm{Nef}^{143}$, A. Negri ${ }^{121 a, 121 b}$, M. Negrini ${ }^{20 a}$, S. Nektarijevic ${ }^{106}$, C. Nellist ${ }^{117}$, A. Nelson ${ }^{163}$, S. Nemecek ${ }^{127}$, P. Nemethy ${ }^{110}$, A.A. Nepomuceno ${ }^{24 a}$, M. Nessi ${ }^{30, a c}$, M.S. Neubauer ${ }^{165}$, M. Neumann ${ }^{175}$, R.M. Neves ${ }^{110}$, P. Nevski ${ }^{25}$, P.R. Newman ${ }^{18}$, D.H. Nguyen ${ }^{6}$, R.B. Nickerson ${ }^{120}$, R. Nicolaidou ${ }^{136}$, B. Nicquevert ${ }^{30}$, J. Nielsen ${ }^{137}$, N. Nikiforou ${ }^{35}$, A. Nikiforov ${ }^{16}$, V. Nikolaenko ${ }^{130, a b}$, I. Nikolic-Audit ${ }^{80}$, K. Nikolopoulos ${ }^{18}$, J.K. Nilsen ${ }^{119}$, P. Nilsson ${ }^{25}$, Y. Ninomiya ${ }^{155}$, A. Nisati ${ }^{132 a}$, R. Nisius ${ }^{101}$, T. Nobe ${ }^{157}$, M. Nomachi ${ }^{118}$, I. Nomidis ${ }^{29}$, T. Nooney ${ }^{76}$, S. Norberg ${ }^{113}$, M. Nordberg ${ }^{30}$, O. Novgorodova ${ }^{44}$, S. Nowak ${ }^{101}$, M. Nozaki ${ }^{66}$, L. Nozka ${ }^{115}$, K. Ntekas ${ }^{10}$, G. Nunes Hanninger ${ }^{88}$, T. Nunnemann ${ }^{100}$, E. Nurse ${ }^{78}$, F. Nuti ${ }^{88}$, B.J. O'Brien ${ }^{46}$, F. O'grady ${ }^{7}$, D.C. O'Neil ${ }^{142}$, V. O'Shea ${ }^{53}$, F.G. Oakham ${ }^{29, d}$, H. Oberlack ${ }^{101}$, T. Obermann ${ }^{21}$, J. Ocariz ${ }^{80}$, A. Ochi ${ }^{67}$, I. Ochoa ${ }^{78}$, J.P. Ochoa-Ricoux ${ }^{32 a}$, S. Oda ${ }^{70}$, S. Odaka ${ }^{66}$, H. Ogren ${ }^{61}$, A. Oh ${ }^{84}$, S.H. Oh ${ }^{45}$, C.C. Ohm ${ }^{15}$, H. Ohman ${ }^{166}$, H. Oide ${ }^{30}$, W. Okamura ${ }^{118}$, H. Okawa ${ }^{160}$, Y. Okumura ${ }^{31}$, T. Okuyama ${ }^{155}$, A. Olariu ${ }^{26 a}$, S.A. Olivares Pino ${ }^{46}$, D. Oliveira Damazio ${ }^{25}$, E. Oliver Garcia ${ }^{167}$, A. Olszewski ${ }^{39}$, J. Olszowska ${ }^{39}$, A. Onofre ${ }^{126 a, 126 e}$, P.U.E. Onyisis ${ }^{31, q}$, C.J. Oram ${ }^{159 a}$, M.J. Oreglia ${ }^{31}$, Y. Oren ${ }^{153}$, D. Orestano ${ }^{134 a, 134 b}$,

N. Orlando ${ }^{154}$, C. Oropeza Barrera ${ }^{53}$, R.S. Orr ${ }^{158}$, B. Osculati ${ }^{50 a, 50 b}$, R. Ospanov ${ }^{84}$, G. Otero y Garzon ${ }^{27}$, H. Otono ${ }^{70}$, M. Ouchrif ${ }^{135 d}$, E.A. Ouellette ${ }^{169}$, F. Ould-Saada ${ }^{119}$, A. Ouraou ${ }^{136}$, K.P. Oussoren ${ }^{107}$, Q. Ouyang ${ }^{33 a}$, A. Ovcharova ${ }^{15}$, M. Owen ${ }^{53}$, R.E. Owen ${ }^{18}$, V.E. Ozcan ${ }^{19 a}$, N. Ozturk ${ }^{8}$, K. Pachal ${ }^{142}$, A. Pacheco Pages ${ }^{12}$, C. Padilla Aranda ${ }^{12}$, M. Pagáčová ${ }^{48}$, S. Pagan Griso ${ }^{15}$, E. Paganis ${ }^{139}$, C. Pahl ${ }^{101}$, F. Paige ${ }^{25}$, P. Pais ${ }^{86}$, K. Pajchel $^{119}$, G. Palacino ${ }^{159 b}$, S. Palestini ${ }^{30}$, M. Palka ${ }^{38 b}$, D. Pallin ${ }^{34}$, A. Palma ${ }^{126 a, 126 b}$, Y.B. Pan ${ }^{173}$, E. Panagiotopoulou ${ }^{10}$, C.E. Pandini ${ }^{80}$, J.G. Panduro Vazquez ${ }^{77}$, P. Pani ${ }^{146 a, 146 b}$, S. Panitkin ${ }^{25}$, D. Pantea ${ }^{26 a}$, L. Paolozzi ${ }^{49}$, Th.D. Papadopoulou ${ }^{10}$, K. Papageorgiou ${ }^{154}$, A. Paramonov ${ }^{6}$, D. Paredes Hernandez ${ }^{154}$, M.A. Parker ${ }^{28}$, K.A. Parker ${ }^{139}$, F. Parodi ${ }^{50 a, 50 b}$, J.A. Parsons ${ }^{35}$, U. Parzefall ${ }^{48}$, E. Pasqualucci ${ }^{132 a}$, S. Passaggio ${ }^{50 a}$, F. Pastore ${ }^{134 a, 134 b, *}$, Fr. Pastore ${ }^{77}$, G. Pásztor ${ }^{29}$, S. Pataraia ${ }^{175}$, N.D. Patel ${ }^{150}$, J.R. Pater ${ }^{84}$, T. Pauly ${ }^{30}$, J. Pearce ${ }^{169}$, B. Pearson ${ }^{113}$, L.E. Pedersen ${ }^{36}$, M. Pedersen ${ }^{119}$, S. Pedraza Lopez ${ }^{167}$, R. Pedro ${ }^{126 a, 126 b}$, S.V. Peleganchuk ${ }^{109, c}$, D. Pelikan ${ }^{166}$, H. Peng ${ }^{33 b}$, B. Penning ${ }^{31}$, J. Penwell ${ }^{61}$, D.V. Perepelitsa ${ }^{25}$, E. Perez Codina ${ }^{159 a}$, M.T. Pérez García-Estañ ${ }^{167}$, L. Perini ${ }^{91 a, 91 b}$, H. Pernegger ${ }^{30}$, S. Perrella ${ }^{104 a, 104 b}$, R. Peschke ${ }^{42}$, V.D. Peshekhonov ${ }^{65}$, K. Peters ${ }^{30}$, R.F.Y. Peters ${ }^{84}$, B.A. Petersen ${ }^{30}$, T.C. Petersen ${ }^{36}$, E. Petit ${ }^{42}$, A. Petridis ${ }^{146 a, 146 b}$, C. Petridou ${ }^{154}$, E. Petrolo ${ }^{132 a}$, F. Petrucci ${ }^{134 a, 134 b}$, N.E. Pettersson ${ }^{157}$, 
R. Pezoa ${ }^{32 b}$, P.W. Phillips ${ }^{131}$, G. Piacquadio ${ }^{143}$, E. Pianori ${ }^{170}$, A. Picazio ${ }^{49}$, E. Piccaro ${ }^{76}$, M. Piccinini ${ }^{20 a}$,20b , M.A. Pickering ${ }^{120}$, R. Piegaia ${ }^{27}$, D.T. Pignotti ${ }^{111}$, J.E. Pilcher ${ }^{31}$,

A.D. Pilkington ${ }^{84}$, J. Pina ${ }^{126 a, 126 b, 126 d}$, M. Pinamonti ${ }^{164 a, 164 c, a d}$, J.L. Pinfold ${ }^{3}$, A. Pingel ${ }^{36}$,

B. Pinto ${ }^{126 a}$, S. Pires $^{80}$, M. Pitt ${ }^{172}$, C. Pizio ${ }^{91 a, 91 b}$, L. Plazak ${ }^{144 a}$, M.-A. Pleier ${ }^{25}$, V. Pleskot ${ }^{129}$,

E. Plotnikova ${ }^{65}$, P. Plucinski ${ }^{146 a, 146 b}$, D. Pluth ${ }^{64}$, R. Poettgen ${ }^{83}$, L. Poggioli ${ }^{117}$, D. Pohl ${ }^{21}$,

G. Polesello ${ }^{121 a}$, A. Policicchio ${ }^{37 a, 37 b}$, R. Polifka ${ }^{158}$, A. Polini ${ }^{20 a}$, C.S. Pollard ${ }^{53}$,

V. Polychronakos ${ }^{25}$, K. Pommès ${ }^{30}$, L. Pontecorvo ${ }^{132 a}$, B.G. Pope ${ }^{90}$, G.A. Popeneciu ${ }^{26 b}$,

D.S. Popovic ${ }^{13}$, A. Poppleton ${ }^{30}$, S. Pospisi ${ }^{128}$, K. Potamianos ${ }^{15}$, I.N. Potrap ${ }^{65}$, C.J. Potter ${ }^{149}$,

C.T. Potter ${ }^{116}$, G. Poulard ${ }^{30}$, J. Poveda ${ }^{30}$, V. Pozdnyakov ${ }^{65}$, P. Pralavorio ${ }^{85}$, A. Pranko ${ }^{15}$,

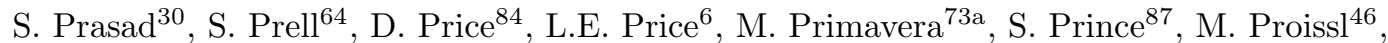

K. Prokofiev ${ }^{60 c}$, F. Prokoshin ${ }^{32 b}$, E. Protopapadaki ${ }^{136}$, S. Protopopescu ${ }^{25}$, J. Proudfoot ${ }^{6}$,

M. Przybycien ${ }^{38 a}$, E. Ptacek ${ }^{116}$, D. Puddu ${ }^{134 a, 134 b}$, E. Pueschel ${ }^{86}$, D. Puldon ${ }^{148}$, M. Purohit ${ }^{25, a e}$,

P. Puzo ${ }^{117}$, J. Qian ${ }^{89}$, G. Qin ${ }^{53}$, Y. Qin ${ }^{84}$, A. Quadt ${ }^{54}$, D.R. Quarrie ${ }^{15}$, W.B. Quayle ${ }^{164 a, 164 b}$,

M. Queitsch-Maitland ${ }^{84}$, D. Quilty ${ }^{53}$, S. Raddum ${ }^{119}$, V. Radeka ${ }^{25}$, V. Radescu ${ }^{42}$,

S.K. Radhakrishnan ${ }^{148}$, P. Radloff ${ }^{116}$, P. $\operatorname{Rados}^{88}$, F. Ragusa ${ }^{91 a, 91 b}$, G. Rahal ${ }^{178}$,

S. Rajagopalan ${ }^{25}$, M. Rammensee ${ }^{30}$, C. Rangel-Smith ${ }^{166}$, F. Rauscher ${ }^{100}$, S. Rave ${ }^{83}$,

T. Ravenscroft ${ }^{53}$, M. Raymond ${ }^{30}$, A.L. Read ${ }^{119}$, N.P. Readioff ${ }^{74}$, D.M. Rebuzzi ${ }^{121 a, 121 b}$,

A. Redelbach ${ }^{174}$, G. Redlinger ${ }^{25}$, R. Reece ${ }^{137}$, K. Reeves ${ }^{41}$, L. Rehnisch ${ }^{16}$, H. Reisin ${ }^{27}$,

M. Relich ${ }^{163}$, C. Rembser ${ }^{30}$, H. Ren ${ }^{33 a}$, A. Renaud ${ }^{117}$, M. Rescigno ${ }^{132 a}$, S. Resconi ${ }^{91 a}$,

O.L. Rezanova ${ }^{109, c}$, P. Reznicek ${ }^{129}$, R. Rezvani ${ }^{95}$, R. Richter ${ }^{101}$, S. Richter ${ }^{78}$, E. Richter-Was ${ }^{38 b}$,

O. Ricken ${ }^{21}$, M. Ridel ${ }^{80}$, P. Rieck ${ }^{16}$, C.J. Riegel ${ }^{175}$, J. Rieger ${ }^{54}$, M. Rijssenbeek ${ }^{148}$,

A. Rimoldi ${ }^{121 a, 121 b}$, L. Rinaldi ${ }^{20 a}$, B. Ristić ${ }^{49}$, E. Ritsch ${ }^{62}$, I. Riu ${ }^{12}$, F. Rizatdinova ${ }^{114}$, E. Rizvi $^{76}$, S.H. Robertson ${ }^{87, k}$, A. Robichaud-Veronneau ${ }^{87}$, D. Robinson ${ }^{28}$, J.E.M. Robinson ${ }^{84}$, A. Robson ${ }^{53}$, C. Roda ${ }^{124 a, 124 b}$, S. Roe ${ }^{30}$, O. Røhne ${ }^{119}$, S. Rolli ${ }^{161}$, A. Romaniouk ${ }^{98}$, M. Romano ${ }^{20 a, 20 b}$, S.M. Romano $\mathrm{Saez}^{34}$, E. Romero Adam ${ }^{167}$, N. Rompotis ${ }^{138}$, M. Ronzani ${ }^{48}$, L. Roos ${ }^{80}$, E. Ros ${ }^{167}$, S. Rosati ${ }^{132 a}$, K. Rosbach ${ }^{48}$, P. Rose ${ }^{137}$, P.L. Rosendahl ${ }^{14}$, O. Rosenthal ${ }^{141}$, V. Rossetti ${ }^{146 a, 146 b}$, E. Rossi ${ }^{104 a, 104 b}$, L.P. Rossi ${ }^{50 a}$, R. Rosten ${ }^{138}$, M. Rotaru ${ }^{26 a}$, I. Roth ${ }^{172}$, J. Rothberg ${ }^{138}$, D. Rousseau ${ }^{117}$, C.R. Royon ${ }^{136}$, A. Rozanov ${ }^{85}$, Y. Rozen ${ }^{152}$, X. Ruan ${ }^{145 c}$, F. Rubbo ${ }^{143}$, I. Rubinskiy ${ }^{42}$, V.I. Rud ${ }^{99}$, C. Rudolph ${ }^{44}$, M.S. Rudolph ${ }^{158}$, F. Rühr ${ }^{48}$, A. Ruiz-Martinez ${ }^{30}$,

Z. Rurikova ${ }^{48}$, N.A. Rusakovich ${ }^{65}$, A. Ruschke ${ }^{100}$, H.L. Russell ${ }^{138}$, J.P. Rutherfoord ${ }^{7}$,

N. Ruthmann ${ }^{48}$, Y.F. Ryabov ${ }^{123}$, M. Rybar ${ }^{129}$, G. Rybkin ${ }^{117}$, N.C. Ryder ${ }^{120}$, A.F. Saavedra ${ }^{150}$,

G. Sabato ${ }^{107}$, S. Sacerdoti ${ }^{27}$, A. Saddique ${ }^{3}$, H.F-W. Sadrozinski ${ }^{137}$, R. Sadykov ${ }^{65}$,

F. Safai Tehrani ${ }^{132 a}$, M. Saimpert ${ }^{136}$, H. Sakamoto ${ }^{155}$, Y. Sakurai ${ }^{171}$, G. Salamanna ${ }^{134 a, 134 b}$,

A. Salamon ${ }^{133 a}$, M. Saleem ${ }^{113}$, D. Salek ${ }^{107}$, P.H. Sales De Bruin ${ }^{138}$, D. Salihagic ${ }^{101}$,

A. Salnikov ${ }^{143}$, J. Salt ${ }^{167}$, D. Salvatore ${ }^{37 a, 37 b}$, F. Salvatore ${ }^{149}$, A. Salvucci ${ }^{106}$, A. Salzburger ${ }^{30}$,

D. Sampsonidis ${ }^{154}$, A. Sanchez ${ }^{104 a, 104 b}$, J. Sánchez ${ }^{167}$, V. Sanchez Martinez ${ }^{167}$, H. Sandaker ${ }^{14}$,

R.L. Sandbach ${ }^{76}$, H.G. Sander ${ }^{83}$, M.P. Sanders ${ }^{100}$, M. Sandhoff ${ }^{175}$, C. Sandoval ${ }^{162}$,

R. Sandstroem ${ }^{101}$, D.P.C. Sankey ${ }^{131}$, M. Sannino ${ }^{50 a, 50 b}$, A. Sansoni ${ }^{47}$, C. Santoni ${ }^{34}$,

R. Santonico ${ }^{133 a, 133 b}$, H. Santos ${ }^{126 a}$, I. Santoyo Castillo ${ }^{149}$, K. Sapp ${ }^{125}$, A. Sapronov ${ }^{65}$,

J.G. Saraiva ${ }^{126 a, 126 d}$, B. Sarrazin ${ }^{21}$, O. Sasaki ${ }^{66}$, Y. Sasaki ${ }^{155}$, K. Sato ${ }^{160}$, G. Sauvage ${ }^{5, *}$,

E. Sauvan ${ }^{5}$, G. Savage ${ }^{77}$, P. Savard ${ }^{158, d}$, C. Sawyer ${ }^{120}$, L. Sawyer ${ }^{79, n}$, J. Saxon ${ }^{31}$, C. Sbarra ${ }^{20 a}$,

A. Sbrizzi ${ }^{20 a, 20 b}$, T. Scanlon ${ }^{78}$, D.A. Scannicchio ${ }^{163}$, M. Scarcella ${ }^{150}$, V. Scarfone ${ }^{37 a, 37 b}$,

J. Schaarschmidt ${ }^{172}$, P. Schacht ${ }^{101}$, D. Schaefer ${ }^{30}$, R. Schaefer ${ }^{42}$, J. Schaeffer ${ }^{83}$, S. Schaepe ${ }^{21}$,

S. Schaetzel ${ }^{58 b}$, U. Schäfer ${ }^{83}$, A.C. Schaffer ${ }^{117}$, D. Schaile ${ }^{100}$, R.D. Schamberger ${ }^{148}$, V. Scharf ${ }^{58 a}$, V.A. Schegelsky ${ }^{123}$, D. Scheirich ${ }^{129}$, M. Schernau ${ }^{163}$, C. Schiavi ${ }^{50 a, 50 b}$, C. Schillo ${ }^{48}$,

M. Schioppa ${ }^{37 a, 37 b}$, S. Schlenker ${ }^{30}$, E. Schmidt ${ }^{48}$, K. Schmieden ${ }^{30}$, C. Schmitt ${ }^{83}$, S. Schmitt ${ }^{58 b}$,

S. Schmitt ${ }^{42}$, B. Schneider ${ }^{159 a}$, Y.J. Schnellbach ${ }^{74}$, U. Schnoor ${ }^{44}$, L. Schoeffel ${ }^{136}$, A. Schoening ${ }^{58 b}$, B.D. Schoenrock ${ }^{90}$, E. Schopf ${ }^{21}$, A.L.S. Schorlemmer ${ }^{54}$, M. Schott ${ }^{83}$, D. Schouten ${ }^{159 a}$, 
J. Schovancova ${ }^{8}$, S. Schramm ${ }^{158}$, M. Schreyer ${ }^{174}$, C. Schroeder ${ }^{83}$, N. Schuh ${ }^{83}$, M.J. Schultens ${ }^{21}$, H.-C. Schultz-Coulon ${ }^{58 a}$, H. Schulz ${ }^{16}$, M. Schumacher ${ }^{48}$, B.A. Schumm ${ }^{137}$, Ph. Schune ${ }^{136}$, C. Schwanenberger ${ }^{84}$, A. Schwartzman ${ }^{143}$, T.A. Schwarz ${ }^{89}$, Ph. Schwegler ${ }^{101}$, Ph. Schwemling ${ }^{136}$, R. Schwienhorst ${ }^{90}$, J. Schwindling ${ }^{136}$, T. Schwindt ${ }^{21}$, M. Schwoerer ${ }^{5}$, F.G. Sciacca ${ }^{17}$, E. Scifo ${ }^{117}$, G. Sciolla ${ }^{23}$, F. Scuri ${ }^{124 a, 124 b}$, F. Scutti ${ }^{21}$, J. Searcy ${ }^{89}$, G. Sedov ${ }^{42}$, E. Sedykh ${ }^{123}$, P. Seema ${ }^{21}$, S.C. Seidel ${ }^{105}$, A. Seiden ${ }^{137}$, F. Seifert ${ }^{128}$, J.M. Seixas ${ }^{24 a}$, G. Sekhniaidze ${ }^{104 a}$, K. Sekhon ${ }^{89}$, S.J. Sekula ${ }^{40}$, K.E. Selbach ${ }^{46}$, D.M. Seliverstov ${ }^{123, *}$, N. Semprini-Cesariina, 20b ${ }^{20}$ C. Serfon ${ }^{30}$, L. Serin ${ }^{117}$, L. Serkin ${ }^{164 a, 164 b}$, T. Serre ${ }^{85}$, M. Sessa ${ }^{134 a, 134 b}$, R. Seuster ${ }^{159 a}$, H. Severini ${ }^{113}$, T. Sfiligoj ${ }^{75}$, F. Sforza ${ }^{101}$, A. Sfyrla ${ }^{30}$, E. Shabalina ${ }^{54}$, M. Shamim ${ }^{116}$, L.Y. Shan ${ }^{33 a}$, R. Shang ${ }^{165}$, J.T. Shank ${ }^{22}$, M. Shapiro ${ }^{15}$, P.B. Shatalov ${ }^{97}$, K. Shaw ${ }^{164 a, 164 b}$, S.M. Shaw ${ }^{84}$, A. Shcherbakova ${ }^{146 a, 146 b}$, C.Y. Shehu ${ }^{149}$, P. Sherwood ${ }^{78}$, L. Shi $^{151, a f}$, S. Shimizu ${ }^{67}$, C.O. Shimmin ${ }^{163}$, M. Shimojima ${ }^{102}$, M. Shiyakova ${ }^{65}$, A. Shmeleva ${ }^{96}$, D. Shoaleh Saadi ${ }^{95}$, M.J. Shochet ${ }^{31}$, S. Shojaii ${ }^{91 a, 91 b}$, S. Shrestha ${ }^{111}$, E. Shulga ${ }^{98}$, M.A. Shupe ${ }^{7}$, S. Shushkevich ${ }^{42}$, P. Sicho ${ }^{127}$, O. Sidiropoulou ${ }^{174}$, D. Sidorov ${ }^{114}$, A. Sidotin ${ }^{20 a}, 20 \mathrm{~b}$, F. Siegert ${ }^{44}$, Dj. Sijacki ${ }^{13}$, J. Silva ${ }^{126 a, 126 d}$, Y. Silver ${ }^{153}$, S.B. Silverstein ${ }^{146 a}$, V. Simak ${ }^{128}$, O. Simard ${ }^{5}$, Lj. Simic ${ }^{13}$, S. Simion ${ }^{117}$, E. Simioni ${ }^{83}$, B. Simmons ${ }^{78}$, D. Simon ${ }^{34}$, R. Simoniello ${ }^{91 a, 91 b}$, P. Sinervo ${ }^{158}$, N.B. Sinev ${ }^{116}$, G. Siragusa ${ }^{174}$, A.N. Sisakyan ${ }^{65, *}$, S.Yu. Sivoklokov ${ }^{99}$, J. Sjölin ${ }^{146 a, 146 b}$, T.B. Sjursen ${ }^{14}$, M.B. Skinner ${ }^{72}$, H.P. Skottowe ${ }^{57}$, P. Skubic ${ }^{113}$, M. Slater ${ }^{18}$, T. Slavicek ${ }^{128}$, M. Slawinska ${ }^{107}$, K. Sliwa ${ }^{161}$, V. Smakhtin ${ }^{172}$, B.H. Smart ${ }^{46}$, L. Smestad ${ }^{14}$, S.Yu. Smirnov ${ }^{98}$, Y. Smirnov ${ }^{98}$, L.N. Smirnova ${ }^{99, a g}$, O. Smirnova ${ }^{81}$, M.N.K. Smith ${ }^{35}$, M. Smizanska ${ }^{72}$, K. Smolek ${ }^{128}$, A.A. Snesarev ${ }^{96}$, G. Snidero ${ }^{76}$, S. Snyder ${ }^{25}$, R. Sobie ${ }^{169, k}$, F. Socher ${ }^{44}$, A. Soffer ${ }^{153}$, D.A. Soh ${ }^{151, a f}$, C.A. Solans ${ }^{30}$, M. Solar ${ }^{128}$, J. Solc ${ }^{128}$, E.Yu. Soldatov ${ }^{98}$, U. Soldevila ${ }^{167}$, A.A. Solodkov ${ }^{130}$, A. Soloshenko ${ }^{65}$, O.V. Solovyanov ${ }^{130}$, V. Solovyev ${ }^{123}$, P. Sommer ${ }^{48}$, H.Y. Song ${ }^{33 b}$, N. Soni ${ }^{1}$, A. Sood ${ }^{15}$, A. Sopczak ${ }^{128}$, B. Sopko ${ }^{128}$, V. Sopko ${ }^{128}$, V. Sorin ${ }^{12}$, D. Sosa ${ }^{58 b}$, M. Sosebee ${ }^{8}$, C.L. Sotiropoulou ${ }^{124 a, 124 b}$, R. Soualah ${ }^{164 a, 164 c}$, P. Soueid ${ }^{95}$, A.M. Soukharev ${ }^{109, c}$, D. South ${ }^{42}$, S. Spagnolo ${ }^{73 a, 73 b}$, M. Spalla ${ }^{124 a, 124 b}$, F. Spanò ${ }^{77}$, W.R. Spearman ${ }^{57}$, F. Spettel ${ }^{101}$, R. Spighi ${ }^{20 a}$, G. Spigo $^{30}$, L.A. Spiller ${ }^{88}$, M. Spousta ${ }^{129}$, T. Spreitzer ${ }^{158}$, R.D. St. Denis ${ }^{53, *}$, S. Staerz ${ }^{44}$, J. Stahlman ${ }^{122}$, R. Stamen ${ }^{58 a}$, S. Stamm ${ }^{16}$, E. Stanecka ${ }^{39}$, C. Stanescu ${ }^{134 a}$, M. Stanescu-Bellu ${ }^{42}$, M.M. Stanitzki ${ }^{42}$, S. Stapnes ${ }^{119}$, E.A. Starchenko ${ }^{130}$, J. Stark ${ }^{55}$, P. Staroba ${ }^{127}$, P. Starovoitov ${ }^{42}$, R. Staszewski ${ }^{39}$, P. Stavina ${ }^{144 a, *}$, P. Steinberg ${ }^{25}$, B. Stelzer ${ }^{142}$, H.J. Stelzer ${ }^{30}$, O. Stelzer-Chilton ${ }^{159 a}$, H. Stenzel ${ }^{52}$, S. Stern ${ }^{101}$, G.A. Stewart ${ }^{53}$, J.A. Stillings ${ }^{21}$, M.C. Stockton ${ }^{87}$, M. Stoebe ${ }^{87}$, G. Stoicea ${ }^{26 a}$, P. Stolte ${ }^{54}$, S. Stonjek ${ }^{101}$, A.R. Stradling ${ }^{8}$, A. Straessner ${ }^{44}$, M.E. Stramaglia ${ }^{17}$, J. Strandberg ${ }^{147}$, S. Strandberg ${ }^{146 a, 146 b}$ A. Strandlie ${ }^{119}$, E. Strauss ${ }^{143}$, M. Strauss ${ }^{113}$, P. Strizenec ${ }^{144 b}$, R. Ströhmer ${ }^{174}$, D.M. Strom ${ }^{116}$, R. Stroynowski ${ }^{40}$, A. Strubig ${ }^{106}$, S.A. Stucci ${ }^{17}$, B. Stugu ${ }^{14}$, N.A. Styles ${ }^{42}$, D. $\mathrm{Su}^{143}$, J. Su ${ }^{125}$, R. Subramaniam ${ }^{79}$, A. Succurro ${ }^{12}$, Y. Sugaya ${ }^{118}$, C. Suhr ${ }^{108}$, M. Suk ${ }^{128}$, V.V. Sulin ${ }^{96}$, S. Sultansoy ${ }^{4 d}$, T. Sumida ${ }^{68}$, S. Sun ${ }^{57}$, X. Sun ${ }^{33 a}$, J.E. Sundermann ${ }^{48}$, K. Suruliz ${ }^{149}$, G. Susinno ${ }^{37 a, 37 b}$, M.R. Sutton ${ }^{149}$, S. Suzuki ${ }^{66}$, Y. Suzuki ${ }^{66}$, M. Svatos ${ }^{127}$, S. Swedish ${ }^{168}$, M. Swiatlowski ${ }^{143}$, I. Sykora ${ }^{144 a}$, T. Sykora ${ }^{129}$, D. Ta ${ }^{90}$, C. Taccini ${ }^{134 a, 134 b}$, K. Tackmann ${ }^{42}$, J. Taenzer ${ }^{158}$, A. Taffard ${ }^{163}$, R. Tafirout ${ }^{159 a}$, N. Taiblum ${ }^{153}$, H. Takai $^{25}$, R. Takashima ${ }^{69}$, H. Takeda ${ }^{67}$, T. Takeshita ${ }^{140}$, Y. Takubo $^{66}$, M. Talby ${ }^{85}$, A.A. Talyshev ${ }^{109, c}$, J.Y.C. Tam ${ }^{174}$, K.G. $\operatorname{Tan}^{88}$, J. Tanaka ${ }^{155}$, R. Tanaka ${ }^{117}$, S. Tanaka ${ }^{66}$, B.B. Tannenwald ${ }^{111}$, N. Tannoury ${ }^{21}$, S. Tapprogge ${ }^{83}$, S. Tarem ${ }^{152}$, F. Tarrade ${ }^{29}$, G.F. Tartarelli ${ }^{91 a}$, P. Tas $^{129}$, M. Tasevsky ${ }^{127}$, T. Tashiro ${ }^{68}$, E. Tassi ${ }^{37 a, 37 b}$, A. Tavares Delgado ${ }^{126 a, 126 b}$, Y. Tayalati ${ }^{135 d}$, F.E. Taylor ${ }^{94}$, G.N. Taylor ${ }^{88}$, W. Taylor ${ }^{159 b}$, F.A. Teischinger ${ }^{30}$, M. Teixeira Dias Castanheira ${ }^{76}$, P. Teixeira-Dias ${ }^{77}$, K.K. Temming ${ }^{48}$, H. Ten Kate ${ }^{30}$, P.K. Teng ${ }^{151}$, J.J. Teoh ${ }^{118}$, F. Tepel ${ }^{175}$,

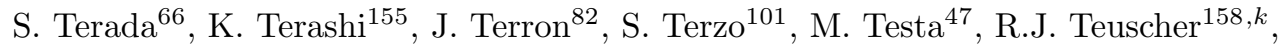
J. Therhaag ${ }^{21}$, T. Theveneaux-Pelzer ${ }^{34}$, J.P. Thomas $^{18}$, J. Thomas-Wilsker ${ }^{77}$, E.N. Thompson ${ }^{35}$, 
P.D. Thompson ${ }^{18}$, R.J. Thompson ${ }^{84}$, A.S. Thompson ${ }^{53}$, L.A. Thomsen ${ }^{176}$, E. Thomson ${ }^{122}$, M. Thomson ${ }^{28}$, R.P. Thun ${ }^{89, *}$, M.J. Tibbetts ${ }^{15}$, R.E. Ticse Torres $^{85}$, V.O. Tikhomirov ${ }^{96, a h}$, Yu.A. Tikhonov ${ }^{109, c}$, S. Timoshenko ${ }^{98}$, E. Tiouchichine ${ }^{85}$, P. Tipton ${ }^{176}$, S. Tisserant ${ }^{85}$,

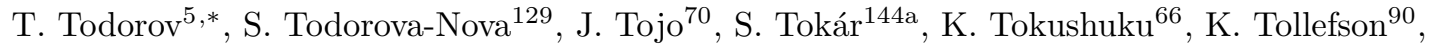
E. Tolley ${ }^{57}$, L. Tomlinson ${ }^{84}$, M. Tomoto ${ }^{103}$, L. Tompkins ${ }^{143, a i}$, K. Toms ${ }^{105}$, E. Torrence $^{116}$, H. Torres ${ }^{142}$, E. Torró Pastor ${ }^{167}$, J. Toth ${ }^{85, a j}$, F. Touchard ${ }^{85}$, D.R. Tovey ${ }^{139}$, T. Trefzger ${ }^{174}$, L. Tremblet ${ }^{30}$, A. Tricoli $^{30}$, I.M. Trigger ${ }^{159 a}$, S. Trincaz-Duvoid ${ }^{80}$, M.F. Tripiana ${ }^{12}$, W. Trischuk ${ }^{158}$, B. Trocmé ${ }^{55}$, C. Troncon ${ }^{91 a}$, M. Trottier-McDonald ${ }^{15}$, M. Trovatelli ${ }^{134 a, 134 b}$, P. True ${ }^{90}$, L. Truong ${ }^{164 a, 164 c}$, M. Trzebinski ${ }^{39}$, A. Trzupek $^{39}$, C. Tsarouchas ${ }^{30}$, J.C-L. Tseng ${ }^{120}$, P.V. Tsiareshka ${ }^{92}$, D. Tsionou ${ }^{154}$, G. Tsipolitis ${ }^{10}$, N. Tsirintanis ${ }^{9}$, S. Tsiskaridze ${ }^{12}$, V. Tsiskaridze ${ }^{48}$, E.G. Tskhadadze ${ }^{51 a}$, I.I. Tsukerman ${ }^{97}$, V. Tsulaia ${ }^{15}$, S. Tsuno ${ }^{66}$, D. Tsybychev ${ }^{148}$, A. Tudorache ${ }^{26 a}$, V. Tudorache ${ }^{26 a}$, A.N. Tuna ${ }^{122}$, S.A. Tupputi20a,20b, S. Turchikhin ${ }^{99, a g}$, D. Turecek ${ }^{128}$, D. Turgeman ${ }^{172}$, R. Turra ${ }^{91 a, 91 b}$, A.J. Turvey ${ }^{40}$, P.M. Tuts ${ }^{35}$,

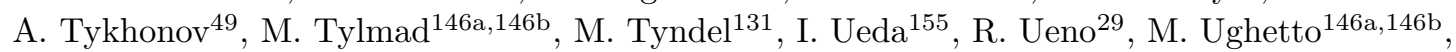
M. Ugland ${ }^{14}$, M. Uhlenbrock ${ }^{21}$, F. Ukegawa ${ }^{160}$, G. Unal ${ }^{30}$, A. Undrus ${ }^{25}$, G. Unel ${ }^{163}$, F.C. Ungaro ${ }^{48}$, Y. Unno ${ }^{66}$, C. Unverdorben ${ }^{100}$, J. Urban ${ }^{144 b}$, P. Urquijo ${ }^{88}$, P. Urrejola ${ }^{83}$,

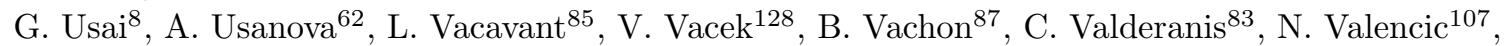
S. Valentinettie ${ }^{20 a}$,20b , A. Valero ${ }^{167}$, L. Valery ${ }^{12}$, S. Valkar ${ }^{129}$, E. Valladolid Gallego ${ }^{167}$, S. Vallecorsa ${ }^{49}$, J.A. Valls Ferrer ${ }^{167}$, W. Van Den Wollenberg ${ }^{107}$, P.C. Van Der Deij1 ${ }^{107}$, R. van der Geer ${ }^{107}$, H. van der Graaf ${ }^{107}$, R. Van Der Leeuw ${ }^{107}$, N. van Eldik ${ }^{152}$, P. van Gemmeren ${ }^{6}$, J. Van Nieuwkoop ${ }^{142}$, I. van Vulpen ${ }^{107}$, M.C. van Woerden ${ }^{30}$, M. Vanadia ${ }^{132 a, 132 b}$, W. Vandelli ${ }^{30}$, R. Vanguri ${ }^{122}$, A. Vaniachine ${ }^{6}$, F. Vannucci ${ }^{80}$, G. Vardanyan ${ }^{177}$, R. Vari ${ }^{132 a}$, E.W. Varnes ${ }^{7}$, T. $\operatorname{Varol}^{40}$, D. Varouchas ${ }^{80}$, A. Vartapetian ${ }^{8}$, K.E. Varvell ${ }^{150}$, F. Vazeille ${ }^{34}$, T. Vazquez Schroeder ${ }^{87}$, J. Veatch ${ }^{7}$, F. Veloso ${ }^{126 a, 126 c}$, T. Velz ${ }^{21}$, S. Veneziano ${ }^{132 a}$, A. Ventura ${ }^{73 a, 73 b}$, D. Ventura ${ }^{86}$, M. Venturi ${ }^{169}$, N. Venturi ${ }^{158}$, A. Venturini ${ }^{23}$, V. Vercesi ${ }^{121 a}$, M. Verducci ${ }^{132 a, 132 b}$, W. Verkerke ${ }^{107}$, J.C. Vermeulen ${ }^{107}$, A. Vest ${ }^{44}$, M.C. Vetterli ${ }^{142, d}$, O. Viazlo ${ }^{81}$, I. Vichou ${ }^{165}$, T. Vickey ${ }^{139}$, O.E. Vickey Boeriu ${ }^{139}$, G.H.A. Viehhauser ${ }^{120}$, S. Viel ${ }^{15}$, R. Vigne ${ }^{30}$, M. Villa ${ }^{20 a, 20 b}$, M. Villaplana Perez ${ }^{91 a, 91 b}$, E. Vilucchi ${ }^{47}$, M.G. Vincter ${ }^{29}$, V.B. Vinogradov ${ }^{65}$, I. Vivarellii ${ }^{149}$, F. Vives Vaque $^{3}$, S. Vlachos $^{10}$, D. Vladoiu ${ }^{100}$, M. Vlasak ${ }^{128}$, M. Vogel ${ }^{32 a}$, P. Vokac ${ }^{128}$, G. Volpi ${ }^{124 a, 124 b}$, M. Volpi ${ }^{88}$, H. von der Schmitt ${ }^{101}$, H. von Radziewski ${ }^{48}$, E. von Toerne ${ }^{21}$, V. Vorobel $^{129}$, K. Vorobev ${ }^{98}$,

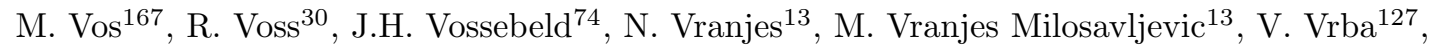
M. Vreeswijk ${ }^{107}$, R. Vuillermet ${ }^{30}$, I. Vukotic ${ }^{31}$, Z. Vykydal ${ }^{128}$, P. Wagner ${ }^{21}$, W. Wagner ${ }^{175}$, H. Wahlberg ${ }^{71}$, S. Wahrmund ${ }^{44}$, J. Wakabayashi ${ }^{103}$, J. Walder ${ }^{72}$, R. Walker ${ }^{100}$, W. Walkowiak ${ }^{141}$, C. Wang ${ }^{33 c}$, F. Wang ${ }^{173}$, H. Wang ${ }^{15}$, H. Wang ${ }^{40}$, J. Wang ${ }^{42}$, J. Wang ${ }^{33 a}$, K. Wang ${ }^{87}$, R. Wang ${ }^{6}$, S.M. Wang ${ }^{151}$, T. Wang ${ }^{21}$, X. Wang ${ }^{176}$, C. Wanotayaroj ${ }^{116}$, A. Warburton ${ }^{87}$, C.P. Ward ${ }^{28}$, D.R. Wardrope ${ }^{78}$, M. Warsinsky ${ }^{48}$, A. Washbrook ${ }^{46}$, C. Wasicki ${ }^{42}$, P.M. Watkins ${ }^{18}$, A.T. Watson ${ }^{18}$, I.J. Watson ${ }^{150}$, M.F. Watson ${ }^{18}$, G. Watts ${ }^{138}$, S. Watts ${ }^{84}$, B.M. Waugh ${ }^{78}$, S. Webb ${ }^{84}$, M.S. Weber ${ }^{17}$, S.W. Weber ${ }^{174}$, J.S. Webster ${ }^{31}$, A.R. Weidberg ${ }^{120}$, B. Weinert ${ }^{61}$, J. Weingarten ${ }^{54}$, C. Weiser ${ }^{48}$, H. Weits ${ }^{107}$, P.S. Wells ${ }^{30}$, T. Wenaus ${ }^{25}$, T. Wengler ${ }^{30}$, S. Wenig ${ }^{30}$, N. Wermes ${ }^{21}$, M. Werner ${ }^{48}$, P. Werner ${ }^{30}$, M. Wessels ${ }^{58 a}$, J. Wetter ${ }^{161}$, K. Whalen ${ }^{29}$, A.M. Wharton ${ }^{72}$, A. White ${ }^{8}$, M.J. White ${ }^{1}$, R. White ${ }^{32 b}$, S. White ${ }^{124 a, 124 b}$, D. Whiteson ${ }^{163}$, F.J. Wickens ${ }^{131}$, W. Wiedenmann ${ }^{173}$, M. Wielers ${ }^{131}$, P. Wienemann ${ }^{21}$, C. Wiglesworth ${ }^{36}$, L.A.M. Wiik-Fuchs ${ }^{21}$, A. Wildauer ${ }^{101}$, H.G. Wilkens ${ }^{30}$, H.H. Williams ${ }^{122}$, S. Williams ${ }^{107}$, C. Willis ${ }^{90}$, S. Willocq ${ }^{86}$, A. Wilson ${ }^{89}$, J.A. Wilson ${ }^{18}$, I. Wingerter-Seez ${ }^{5}$, F. Winklmeier ${ }^{116}$, B.T. Winter ${ }^{21}$, M. Wittgen ${ }^{143}$, J. Wittkowski ${ }^{100}$, S.J. Wollstadt ${ }^{83}$, M.W. Wolter ${ }^{39}$, H. Wolters ${ }^{126 a, 126 c}$, B.K. Wosiek ${ }^{39}$, J. Wotschack ${ }^{30}$, M.J. Woudstra ${ }^{84}$, K.W. Wozniak ${ }^{39}$, M. Wu $^{55}$, M. $\mathrm{Wu}^{31}$, S.L. $\mathrm{Wu}^{173}$, X. $\mathrm{Wu}^{49}$, Y. $\mathrm{Wu}^{89}$, T.R. Wyatt ${ }^{84}$, B.M. Wynne ${ }^{46}$, S. Xella ${ }^{36}$, D. Xu ${ }^{33 a}$, 
L. Xu ${ }^{33 b, a k}$, B. Yabsley ${ }^{150}$, S. Yacoob ${ }^{145 b, a l}$, R. Yakabe ${ }^{67}$, M. Yamada ${ }^{66}$, Y. Yamaguchi ${ }^{118}$, A. Yamamoto ${ }^{66}$, S. Yamamoto ${ }^{155}$, T. Yamanaka ${ }^{155}$, K. Yamauchi ${ }^{103}$, Y. Yamazaki ${ }^{67}$, Z. Yan $^{22}$,

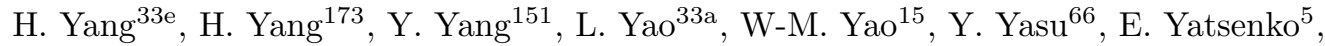
K.H. Yau Wong ${ }^{21}$, J. Ye ${ }^{40}, \mathrm{~S} . \mathrm{Ye}^{25}$, I. Yeletskikh ${ }^{65}$, A.L. Yen ${ }^{57}$, E. Yildirim ${ }^{42}$, K. Yorita ${ }^{171}$, R. Yoshida ${ }^{6}$, K. Yoshihara ${ }^{122}$, C. Young ${ }^{143}$, C.J.S. Young ${ }^{30}$, S. Youssef ${ }^{22}$, D.R. Yu ${ }^{15}$, J. Yu $^{8}$, J.M. Yu ${ }^{89}$, J. Yu ${ }^{114}$, L. Yuan ${ }^{67}$, A. Yurkewicz ${ }^{108}$, I. Yusuff ${ }^{28, a m}$, B. Zabinski ${ }^{39}$, R. Zaidan ${ }^{63}$, A.M. Zaitsev ${ }^{130, a b}$, J. Zalieckas ${ }^{14}$, A. Zaman ${ }^{148}$, S. Zambito57, L. Zanello132a,132b , D. Zanzi ${ }^{88}$, C. Zeitnitz ${ }^{175}$, M. Zeman ${ }^{128}$, A. Zemla ${ }^{38 a}$, K. Zengel $^{23}$, O. Zenin ${ }^{130}$, T. Ženiš ${ }^{144 a}$, D. Zerwas ${ }^{117}$, D. Zhang ${ }^{89}$, F. Zhang ${ }^{173}$, J. Zhang ${ }^{6}$, L. Zhang ${ }^{48}$, R. Zhang ${ }^{33 b}$, X. Zhang ${ }^{33 d}$, Z. Zhang ${ }^{117}$, X. Zhao ${ }^{40}$, Y. Zhao ${ }^{33 d, 117}$, Z. Zhao ${ }^{33 b}$, A. Zhemchugov ${ }^{65}$, J. Zhong ${ }^{120}$, B. Zhou ${ }^{89}$, C. Zhou ${ }^{45}$, L. Zhou ${ }^{35}$, L. Zhou ${ }^{40}$, N. Zhou ${ }^{163}$, C.G. Zhu ${ }^{33 d}$, H. Zhu ${ }^{33 a}$, J. Zhu ${ }^{89}$, Y. Zhu ${ }^{33 b}$, X. Zhuang ${ }^{33 a}$, K. Zhukov ${ }^{96}$, A. Zibell ${ }^{174}$, D. Zieminska ${ }^{61}$, N.I. Zimine ${ }^{65}$, C. Zimmermann ${ }^{83}$, S. Zimmermann ${ }^{48}$, Z. Zinonos ${ }^{54}$, M. Zinser ${ }^{83}$, M. Ziolkowski ${ }^{141}$, L. Živković ${ }^{13}$, G. Zobernig ${ }^{173}$, A. Zoccoli ${ }^{20 a, 20 b}$, M. zur Nedden ${ }^{16}$, G. Zurzolo ${ }^{104 a, 104 b}$, L. Zwalinski ${ }^{30}$.

1 Department of Physics, University of Adelaide, Adelaide, Australia

2 Physics Department, SUNY Albany, Albany NY, United States of America

3 Department of Physics, University of Alberta, Edmonton AB, Canada

4 (a) Department of Physics, Ankara University, Ankara; ${ }^{(c)}$ Istanbul Aydin University, Istanbul; ${ }^{(d)}$ Division of Physics, TOBB University of Economics and Technology, Ankara, Turkey

5 LAPP, CNRS/IN2P3 and Université Savoie Mont Blanc, Annecy-le-Vieux, France

6 High Energy Physics Division, Argonne National Laboratory, Argonne IL, United States of America

7 Department of Physics, University of Arizona, Tucson AZ, United States of America

8 Department of Physics, The University of Texas at Arlington, Arlington TX, United States of America

9 Physics Department, University of Athens, Athens, Greece

10 Physics Department, National Technical University of Athens, Zografou, Greece

11 Institute of Physics, Azerbaijan Academy of Sciences, Baku, Azerbaijan

12 Institut de Física d'Altes Energies and Departament de Física de la Universitat Autònoma de Barcelona, Barcelona, Spain

13 Institute of Physics, University of Belgrade, Belgrade, Serbia

14 Department for Physics and Technology, University of Bergen, Bergen, Norway

15 Physics Division, Lawrence Berkeley National Laboratory and University of California, Berkeley CA, United States of America

16 Department of Physics, Humboldt University, Berlin, Germany

17 Albert Einstein Center for Fundamental Physics and Laboratory for High Energy Physics, University of Bern, Bern, Switzerland

18 School of Physics and Astronomy, University of Birmingham, Birmingham, United Kingdom

19 (a) Department of Physics, Bogazici University, Istanbul; ${ }^{(b)}$ Department of Physics, Dogus University, Istanbul; ${ }^{(c)}$ Department of Physics Engineering, Gaziantep University, Gaziantep, Turkey

20 (a) INFN Sezione di Bologna; ${ }^{(b)}$ Dipartimento di Fisica e Astronomia, Università di Bologna, Bologna, Italy

21 Physikalisches Institut, University of Bonn, Bonn, Germany

22 Department of Physics, Boston University, Boston MA, United States of America

23 Department of Physics, Brandeis University, Waltham MA, United States of America

24 (a) Universidade Federal do Rio De Janeiro COPPE/EE/IF, Rio de Janeiro; ${ }^{(b)}$ Electrical Circuits Department, Federal University of Juiz de Fora (UFJF), Juiz de Fora; ${ }^{(c)}$ Federal University of Sao Joao del Rei (UFSJ), Sao Joao del Rei; ${ }^{(d)}$ Instituto de Fisica, Universidade de Sao Paulo, Sao Paulo, Brazil

25 Physics Department, Brookhaven National Laboratory, Upton NY, United States of America 
26 (a) National Institute of Physics and Nuclear Engineering, Bucharest; ${ }^{(b)}$ National Institute for Research and Development of Isotopic and Molecular Technologies, Physics Department, Cluj Napoca; ${ }^{(c)}$ University Politehnica Bucharest, Bucharest; ${ }^{(d)}$ West University in Timisoara, Timisoara, Romania

27 Departamento de Física, Universidad de Buenos Aires, Buenos Aires, Argentina

28 Cavendish Laboratory, University of Cambridge, Cambridge, United Kingdom

29 Department of Physics, Carleton University, Ottawa ON, Canada

30 CERN, Geneva, Switzerland

31 Enrico Fermi Institute, University of Chicago, Chicago IL, United States of America

32 (a) Departamento de Física, Pontificia Universidad Católica de Chile, Santiago; ${ }^{(b)}$ Departamento de Física, Universidad Técnica Federico Santa María, Valparaíso, Chile

33 (a) Institute of High Energy Physics, Chinese Academy of Sciences, Beijing; ${ }^{(b)}$ Department of Modern Physics, University of Science and Technology of China, Anhui; (c) Department of Physics, Nanjing University, Jiangsu; ${ }^{(d)}$ School of Physics, Shandong University, Shandong; ${ }^{(e)}$ Department of Physics and Astronomy, Shanghai Key Laboratory for Particle Physics and Cosmology, Shanghai Jiao Tong University, Shanghai; ${ }^{(f)}$ Physics Department, Tsinghua University, Beijing 100084, China

34 Laboratoire de Physique Corpusculaire, Clermont Université and Université Blaise Pascal and CNRS/IN2P3, Clermont-Ferrand, France

35 Nevis Laboratory, Columbia University, Irvington NY, United States of America

36 Niels Bohr Institute, University of Copenhagen, Kobenhavn, Denmark

37 (a) INFN Gruppo Collegato di Cosenza, Laboratori Nazionali di Frascati; ${ }^{(b)}$ Dipartimento di Fisica, Università della Calabria, Rende, Italy

38 (a) AGH University of Science and Technology, Faculty of Physics and Applied Computer Science, Krakow; ${ }^{(b)}$ Marian Smoluchowski Institute of Physics, Jagiellonian University, Krakow, Poland

39 Institute of Nuclear Physics Polish Academy of Sciences, Krakow, Poland

40 Physics Department, Southern Methodist University, Dallas TX, United States of America

41 Physics Department, University of Texas at Dallas, Richardson TX, United States of America

42 DESY, Hamburg and Zeuthen, Germany

43 Institut für Experimentelle Physik IV, Technische Universität Dortmund, Dortmund, Germany

44 Institut für Kern- und Teilchenphysik, Technische Universität Dresden, Dresden, Germany

45 Department of Physics, Duke University, Durham NC, United States of America

46 SUPA - School of Physics and Astronomy, University of Edinburgh, Edinburgh, United Kingdom

47 INFN Laboratori Nazionali di Frascati, Frascati, Italy

48 Fakultät für Mathematik und Physik, Albert-Ludwigs-Universität, Freiburg, Germany

49 Section de Physique, Université de Genève, Geneva, Switzerland

50 (a) INFN Sezione di Genova; ${ }^{(b)}$ Dipartimento di Fisica, Università di Genova, Genova, Italy

51 (a) E. Andronikashvili Institute of Physics, Iv. Javakhishvili Tbilisi State University, Tbilisi; ${ }^{(b)}$ High Energy Physics Institute, Tbilisi State University, Tbilisi, Georgia

52 II Physikalisches Institut, Justus-Liebig-Universität Giessen, Giessen, Germany

53 SUPA - School of Physics and Astronomy, University of Glasgow, Glasgow, United Kingdom

54 II Physikalisches Institut, Georg-August-Universität, Göttingen, Germany

55 Laboratoire de Physique Subatomique et de Cosmologie, Université Grenoble-Alpes, CNRS/IN2P3, Grenoble, France

56 Department of Physics, Hampton University, Hampton VA, United States of America

57 Laboratory for Particle Physics and Cosmology, Harvard University, Cambridge MA, United States of America

58 (a) Kirchhoff-Institut für Physik, Ruprecht-Karls-Universität Heidelberg, Heidelberg; ${ }^{(b)}$ Physikalisches Institut, Ruprecht-Karls-Universität Heidelberg, Heidelberg; ${ }^{(c)}$ ZITI Institut für technische Informatik, Ruprecht-Karls-Universität Heidelberg, Mannheim, Germany

59 Faculty of Applied Information Science, Hiroshima Institute of Technology, Hiroshima, Japan

60 (a) Department of Physics, The Chinese University of Hong Kong, Shatin, N.T., Hong Kong; ${ }^{(b)}$ 
Department of Physics, The University of Hong Kong, Hong Kong; ${ }^{(c)}$ Department of Physics, The Hong Kong University of Science and Technology, Clear Water Bay, Kowloon, Hong Kong, China

61 Department of Physics, Indiana University, Bloomington IN, United States of America

62 Institut für Astro- und Teilchenphysik, Leopold-Franzens-Universität, Innsbruck, Austria

63 University of Iowa, Iowa City IA, United States of America

64 Department of Physics and Astronomy, Iowa State University, Ames IA, United States of America

65 Joint Institute for Nuclear Research, JINR Dubna, Dubna, Russia

66 KEK, High Energy Accelerator Research Organization, Tsukuba, Japan

67 Graduate School of Science, Kobe University, Kobe, Japan

68 Faculty of Science, Kyoto University, Kyoto, Japan

69 Kyoto University of Education, Kyoto, Japan

70 Department of Physics, Kyushu University, Fukuoka, Japan

${ }^{71}$ Instituto de Física La Plata, Universidad Nacional de La Plata and CONICET, La Plata, Argentina

72 Physics Department, Lancaster University, Lancaster, United Kingdom

$73{ }^{(a)}$ INFN Sezione di Lecce; ${ }^{(b)}$ Dipartimento di Matematica e Fisica, Università del Salento, Lecce, Italy

74 Oliver Lodge Laboratory, University of Liverpool, Liverpool, United Kingdom

75 Department of Physics, Jožef Stefan Institute and University of Ljubljana, Ljubljana, Slovenia

76 School of Physics and Astronomy, Queen Mary University of London, London, United Kingdom

77 Department of Physics, Royal Holloway University of London, Surrey, United Kingdom

78 Department of Physics and Astronomy, University College London, London, United Kingdom

79 Louisiana Tech University, Ruston LA, United States of America

80 Laboratoire de Physique Nucléaire et de Hautes Energies, UPMC and Université Paris-Diderot and CNRS/IN2P3, Paris, France

81 Fysiska institutionen, Lunds universitet, Lund, Sweden

82 Departamento de Fisica Teorica C-15, Universidad Autonoma de Madrid, Madrid, Spain

83 Institut für Physik, Universität Mainz, Mainz, Germany

84 School of Physics and Astronomy, University of Manchester, Manchester, United Kingdom

85 CPPM, Aix-Marseille Université and CNRS/IN2P3, Marseille, France

86 Department of Physics, University of Massachusetts, Amherst MA, United States of America

87 Department of Physics, McGill University, Montreal QC, Canada

88 School of Physics, University of Melbourne, Victoria, Australia

89 Department of Physics, The University of Michigan, Ann Arbor MI, United States of America

90 Department of Physics and Astronomy, Michigan State University, East Lansing MI, United States of America

91 (a) INFN Sezione di Milano; ${ }^{(b)}$ Dipartimento di Fisica, Università di Milano, Milano, Italy

92 B.I. Stepanov Institute of Physics, National Academy of Sciences of Belarus, Minsk, Republic of Belarus

93 National Scientific and Educational Centre for Particle and High Energy Physics, Minsk, Republic of Belarus

94 Department of Physics, Massachusetts Institute of Technology, Cambridge MA, United States of America

95 Group of Particle Physics, University of Montreal, Montreal QC, Canada

96 P.N. Lebedev Institute of Physics, Academy of Sciences, Moscow, Russia

97 Institute for Theoretical and Experimental Physics (ITEP), Moscow, Russia

98 National Research Nuclear University MEPhI, Moscow, Russia

99 D.V. Skobeltsyn Institute of Nuclear Physics, M.V. Lomonosov Moscow State University, Moscow, Russia

100 Fakultät für Physik, Ludwig-Maximilians-Universität München, München, Germany

101 Max-Planck-Institut für Physik (Werner-Heisenberg-Institut), München, Germany

102 Nagasaki Institute of Applied Science, Nagasaki, Japan

103 Graduate School of Science and Kobayashi-Maskawa Institute, Nagoya University, Nagoya, Japan 
104 (a) INFN Sezione di Napoli; ${ }^{(b)}$ Dipartimento di Fisica, Università di Napoli, Napoli, Italy

Department of Physics and Astronomy, University of New Mexico, Albuquerque NM, United States of America

106 Institute for Mathematics, Astrophysics and Particle Physics, Radboud University Nijmegen/Nikhef, Nijmegen, Netherlands

107 Nikhef National Institute for Subatomic Physics and University of Amsterdam, Amsterdam, Netherlands

108 Department of Physics, Northern Illinois University, DeKalb IL, United States of America

109 Budker Institute of Nuclear Physics, SB RAS, Novosibirsk, Russia

110 Department of Physics, New York University, New York NY, United States of America

111 Ohio State University, Columbus OH, United States of America

112 Faculty of Science, Okayama University, Okayama, Japan

113 Homer L. Dodge Department of Physics and Astronomy, University of Oklahoma, Norman OK, United States of America

114 Department of Physics, Oklahoma State University, Stillwater OK, United States of America

115 Palacký University, RCPTM, Olomouc, Czech Republic

116 Center for High Energy Physics, University of Oregon, Eugene OR, United States of America

117 LAL, Université Paris-Sud and CNRS/IN2P3, Orsay, France

118 Graduate School of Science, Osaka University, Osaka, Japan

119 Department of Physics, University of Oslo, Oslo, Norway

120 Department of Physics, Oxford University, Oxford, United Kingdom

121 (a) INFN Sezione di Pavia; ${ }^{(b)}$ Dipartimento di Fisica, Università di Pavia, Pavia, Italy

122 Department of Physics, University of Pennsylvania, Philadelphia PA, United States of America

123 National Research Centre "Kurchatov Institute" B.P.Konstantinov Petersburg Nuclear Physics Institute, St. Petersburg, Russia

124 (a) INFN Sezione di Pisa; ${ }^{(b)}$ Dipartimento di Fisica E. Fermi, Università di Pisa, Pisa, Italy

125 Department of Physics and Astronomy, University of Pittsburgh, Pittsburgh PA, United States of America

126 (a) Laboratorio de Instrumentacao e Fisica Experimental de Particulas - LIP, Lisboa; ${ }^{(b)}$ Faculdade de Ciências, Universidade de Lisboa, Lisboa; ${ }^{(c)}$ Department of Physics, University of Coimbra, Coimbra; ${ }^{(d)}$ Centro de Física Nuclear da Universidade de Lisboa, Lisboa; ${ }^{(e)}$ Departamento de Fisica, Universidade do Minho, Braga; ${ }^{(f)}$ Departamento de Fisica Teorica y del Cosmos and CAFPE, Universidad de Granada, Granada (Spain); ${ }^{(g)}$ Dep Fisica and CEFITEC of Faculdade de Ciencias e Tecnologia, Universidade Nova de Lisboa, Caparica, Portugal

127 Institute of Physics, Academy of Sciences of the Czech Republic, Praha, Czech Republic

128 Czech Technical University in Prague, Praha, Czech Republic

129 Faculty of Mathematics and Physics, Charles University in Prague, Praha, Czech Republic

130 State Research Center Institute for High Energy Physics, Protvino, Russia

131 Particle Physics Department, Rutherford Appleton Laboratory, Didcot, United Kingdom

132 (a) INFN Sezione di Roma; (b) Dipartimento di Fisica, Sapienza Università di Roma, Roma, Italy

133 (a) INFN Sezione di Roma Tor Vergata; ${ }^{(b)}$ Dipartimento di Fisica, Università di Roma Tor Vergata, Roma, Italy

134 (a) INFN Sezione di Roma Tre; ${ }^{(b)}$ Dipartimento di Matematica e Fisica, Università Roma Tre, Roma, Italy

135 (a) Faculté des Sciences Ain Chock, Réseau Universitaire de Physique des Hautes Energies Université Hassan II, Casablanca; ${ }^{(b)}$ Centre National de l'Energie des Sciences Techniques Nucleaires, Rabat; ${ }^{(c)}$ Faculté des Sciences Semlalia, Université Cadi Ayyad, LPHEA-Marrakech; (d) Faculté des Sciences, Université Mohamed Premier and LPTPM, Oujda; ${ }^{(e)}$ Faculté des sciences, Université Mohammed V-Agdal, Rabat, Morocco

136 DSM/IRFU (Institut de Recherches sur les Lois Fondamentales de l'Univers), CEA Saclay (Commissariat à l'Energie Atomique et aux Energies Alternatives), Gif-sur-Yvette, France

137 Santa Cruz Institute for Particle Physics, University of California Santa Cruz, Santa Cruz CA, 
United States of America

138 Department of Physics, University of Washington, Seattle WA, United States of America

139 Department of Physics and Astronomy, University of Sheffield, Sheffield, United Kingdom

140 Department of Physics, Shinshu University, Nagano, Japan

141 Fachbereich Physik, Universität Siegen, Siegen, Germany

142 Department of Physics, Simon Fraser University, Burnaby BC, Canada

143 SLAC National Accelerator Laboratory, Stanford CA, United States of America

144 (a) Faculty of Mathematics, Physics $\mathcal{E}$ Informatics, Comenius University, Bratislava; ${ }^{(b)}$

Department of Subnuclear Physics, Institute of Experimental Physics of the Slovak Academy of Sciences, Kosice, Slovak Republic

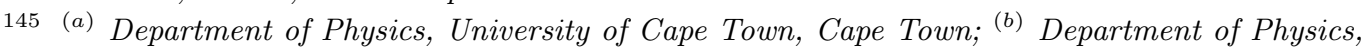
University of Johannesburg, Johannesburg; ${ }^{(c)}$ School of Physics, University of the Witwatersrand, Johannesburg, South Africa

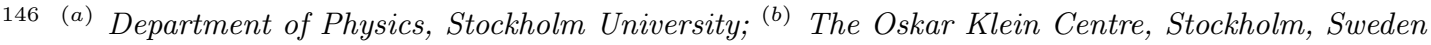

147 Physics Department, Royal Institute of Technology, Stockholm, Sweden

148 Departments of Physics 85 Astronomy and Chemistry, Stony Brook University, Stony Brook NY, United States of America

149 Department of Physics and Astronomy, University of Sussex, Brighton, United Kingdom

150 School of Physics, University of Sydney, Sydney, Australia

151 Institute of Physics, Academia Sinica, Taipei, Taiwan

152 Department of Physics, Technion: Israel Institute of Technology, Haifa, Israel

153 Raymond and Beverly Sackler School of Physics and Astronomy, Tel Aviv University, Tel Aviv, Israel

154 Department of Physics, Aristotle University of Thessaloniki, Thessaloniki, Greece

155 International Center for Elementary Particle Physics and Department of Physics, The University of Tokyo, Tokyo, Japan

156 Graduate School of Science and Technology, Tokyo Metropolitan University, Tokyo, Japan

157 Department of Physics, Tokyo Institute of Technology, Tokyo, Japan

158 Department of Physics, University of Toronto, Toronto ON, Canada

159 (a) TRIUMF, Vancouver BC; ${ }^{(b)}$ Department of Physics and Astronomy, York University, Toronto ON, Canada

160 Faculty of Pure and Applied Sciences, University of Tsukuba, Tsukuba, Japan

161 Department of Physics and Astronomy, Tufts University, Medford MA, United States of America

162 Centro de Investigaciones, Universidad Antonio Narino, Bogota, Colombia

163 Department of Physics and Astronomy, University of California Irvine, Irvine CA, United States of America

164 (a) INFN Gruppo Collegato di Udine, Sezione di Trieste, Udine; ${ }^{(b)}$ ICTP, Trieste; ${ }^{(c)}$ Dipartimento di Chimica, Fisica e Ambiente, Università di Udine, Udine, Italy

165 Department of Physics, University of Illinois, Urbana IL, United States of America

166 Department of Physics and Astronomy, University of Uppsala, Uppsala, Sweden

167 Instituto de Física Corpuscular (IFIC) and Departamento de Física Atómica, Molecular y Nuclear and Departamento de Ingeniería Electrónica and Instituto de Microelectrónica de Barcelona (IMB-CNM), University of Valencia and CSIC, Valencia, Spain

168 Department of Physics, University of British Columbia, Vancouver BC, Canada

169 Department of Physics and Astronomy, University of Victoria, Victoria BC, Canada

170 Department of Physics, University of Warwick, Coventry, United Kingdom

171 Waseda University, Tokyo, Japan

172 Department of Particle Physics, The Weizmann Institute of Science, Rehovot, Israel

173 Department of Physics, University of Wisconsin, Madison WI, United States of America

174 Fakultät für Physik und Astronomie, Julius-Maximilians-Universität, Würzburg, Germany

175 Fachbereich C Physik, Bergische Universität Wuppertal, Wuppertal, Germany

176 Department of Physics, Yale University, New Haven CT, United States of America 
177 Yerevan Physics Institute, Yerevan, Armenia

178 Centre de Calcul de l'Institut National de Physique Nucléaire et de Physique des Particules (IN2P3), Villeurbanne, France

a Also at Department of Physics, King's College London, London, United Kingdom

${ }^{b}$ Also at Institute of Physics, Azerbaijan Academy of Sciences, Baku, Azerbaijan

c Also at Novosibirsk State University, Novosibirsk, Russia

d Also at TRIUMF, Vancouver BC, Canada

e Also at Department of Physics, California State University, Fresno CA, United States of America

${ }^{f}$ Also at Department of Physics, University of Fribourg, Fribourg, Switzerland

$g$ Also at Departamento de Fisica e Astronomia, Faculdade de Ciencias, Universidade do Porto, Portugal

${ }^{h}$ Also at Tomsk State University, Tomsk, Russia

i Also at CPPM, Aix-Marseille Université and CNRS/IN2P3, Marseille, France

$j$ Also at Universita di Napoli Parthenope, Napoli, Italy

${ }^{k}$ Also at Institute of Particle Physics (IPP), Canada

${ }^{l}$ Also at Particle Physics Department, Rutherford Appleton Laboratory, Didcot, United Kingdom

$m$ Also at Department of Physics, St. Petersburg State Polytechnical University, St. Petersburg, Russia

$n$ Also at Louisiana Tech University, Ruston LA, United States of America

- Also at Institucio Catalana de Recerca i Estudis Avancats, ICREA, Barcelona, Spain

$p$ Also at Department of Physics, National Tsing Hua University, Taiwan

$q$ Also at Department of Physics, The University of Texas at Austin, Austin TX, United States of America

${ }^{r}$ Also at Institute of Theoretical Physics, Ilia State University, Tbilisi, Georgia

$s$ Also at CERN, Geneva, Switzerland

${ }^{t}$ Also at Georgian Technical University (GTU), Tbilisi, Georgia

u Also at Ochadai Academic Production, Ochanomizu University, Tokyo, Japan

$v$ Also at Manhattan College, New York NY, United States of America

w Also at Hellenic Open University, Patras, Greece

${ }^{x}$ Also at Institute of Physics, Academia Sinica, Taipei, Taiwan

y Also at LAL, Université Paris-Sud and CNRS/IN2P3, Orsay, France

$z$ Also at Academia Sinica Grid Computing, Institute of Physics, Academia Sinica, Taipei, Taiwan

a Also at School of Physics, Shandong University, Shandong, China

ab Also at Moscow Institute of Physics and Technology State University, Dolgoprudny, Russia

ac Also at section de Physique, Université de Genève, Geneva, Switzerland

ad Also at International School for Advanced Studies (SISSA), Trieste, Italy

ae Also at Department of Physics and Astronomy, University of South Carolina, Columbia SC, United States of America

af Also at School of Physics and Engineering, Sun Yat-sen University, Guangzhou, China

ag Also at Faculty of Physics, M.V.Lomonosov Moscow State University, Moscow, Russia

ah Also at National Research Nuclear University MEPhI, Moscow, Russia

ai Also at Department of Physics, Stanford University, Stanford CA, United States of America

aj Also at Institute for Particle and Nuclear Physics, Wigner Research Centre for Physics, Budapest, Hungary

ak Also at Department of Physics, The University of Michigan, Ann Arbor MI, United States of America

al Also at Discipline of Physics, University of KwaZulu-Natal, Durban, South Africa

am Also at University of Malaya, Department of Physics, Kuala Lumpur, Malaysia

* Deceased 